Marquette University

e-Publications@Marquette

$1-1-2014$

Isomer Dependence in the Assembly and Lability of Silver(I) Trifluoromethanesulfonate Complexes of the Heteroditopic Ligands, 2-, 3-, and 4-[Di( $1 H_{-}$ pyrazolyl)methyl]phenyl(di-p-tolyl)phosphine

James R. Gardinier

MarquetteUniversity, james.gardinier@marquette.edu

Jeewantha S. Hewage

Marquette University, jeewantha.hewage@marquette.edu

Sergey V. Lindeman

Marquette University, sergey.lindeman@marquette.edu

Accepted version. Inorganic Chemistry, Vol. 53, No. 22 (2014): 12108-12121. DOI. (C) 2014

American Chemical Society. Used with permission. 


\title{
Isomer Dependence in the Assembly and Lability of Silver(I) Trifluoromethanesulfonate Complexes of the Heteroditopic Ligands, 2-, 3-, and 4-[Di(1H- pyrazolyl)methyl]phenyl(di-p- tolyl)phosphine
}

\author{
James R. Gardinier \\ Department of Chemistry, Marquette University, \\ Milwaukee, WI \\ Jeewantha S. Hewage \\ Department of Chemistry, Marquette University, \\ Milwaukee, WI \\ Sergey V. Lindeman \\ Department of Chemistry, Marquette University, \\ Milwaukee, WI
}


NOT THE PUBLISHED VERSION; this is the author's final, peer-reviewed manuscript. The published version may be accessed by following the link in the citation at the bottom of the page.

Synopsis: The solid state and solution structures of silver triflate complexes of the three isomers of new $\mathrm{P}, \mathrm{N}-\mathrm{N}$ heteroditopic ligands are examined.

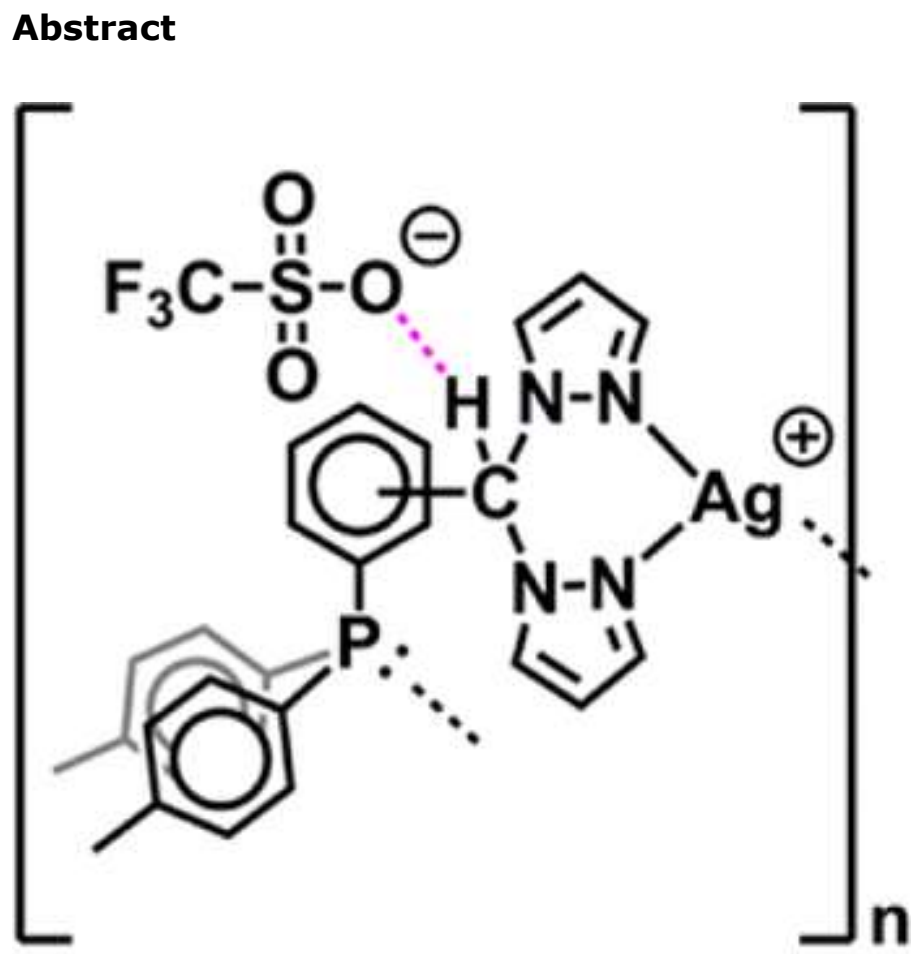

\section{para-, meta-: $\mathbf{n}=\mathbf{2}$ ortho-: $n=\infty$}

Three isomers of a new heteroditopic ligand that contains a di $(1 \mathrm{H}$ pyrazolyl)methyl (- $\left.\mathrm{CHpz}_{2}\right)$ moiety connected to a di( $p$-tolyl)phosphine group via a para-, meta-, or ortho-phenylene spacer $(\boldsymbol{p} \boldsymbol{L}, \boldsymbol{m L}$, and $\boldsymbol{o} \boldsymbol{L}$, respectively) have been synthesized by using a palladium(0)-catalyzed coupling reaction between $\mathrm{HP}(p \text {-tolyl })_{2}$ and the appropriate isomer of $\left(\mathrm{IC}_{6} \mathrm{H}_{4}\right) \mathrm{CHpz}_{2}$. The $1: 1$ complexes of silver(I) trifluoromethanesulfonate, $\mathrm{Ag}$ (OTf), were prepared to examine the nature of ligand coordination and the type of supramolecular isomer (monomeric, cyclic oligomeric, or polymeric) that would be obtained. The single crystal X-ray diffraction studies showed that $[\mathrm{Ag}(\boldsymbol{p} \boldsymbol{L})](\mathrm{OTf}), \mathbf{1}$, and $[\mathrm{Ag}(\boldsymbol{m} \boldsymbol{L})](\mathrm{OTf}), \mathbf{2}$, possessed cyclic dimeric dications, whereas $[\mathrm{Ag}(\boldsymbol{o L})](\mathrm{OTf})$, $\mathbf{3}$, was a coordination polymer. The polymeric chain in $\mathbf{3}$ could be disrupted by reaction with triphenylphosphine, and the resulting complex, $\left[\mathrm{Ag}(\boldsymbol{O L})\left(\mathrm{PPh}_{3}\right)\right](\mathrm{OTf}), \mathbf{4}$, possessed a monometallic cation where the ligand was bound to silver in a chelating $\mathrm{K}^{2} \mathrm{P}, \mathrm{N}$ - coordination mode. The solution structures of 1-4 were probed via a combination of IR, variable-temperature multinuclear $\left({ }^{1} \mathrm{H},{ }^{13} \mathrm{C},{ }^{31} \mathrm{P}\right)$ NMR spectroscopy, as well as by electron spray ionization $(\mathrm{ESI})(+)$ mass spectrometry. A related complex $[\mathrm{Ag}(\mathrm{m}-$ $\left.\left.\mathrm{IC}_{6} \mathrm{H}_{4} \mathrm{CHpz}_{2}\right)_{2}\right](\mathrm{OTf})$, 5, was also prepared, and its solid-state and solution 
spectroscopic properties were studied for comparison purposes. These studies suggest that the cyclic structures of $\mathbf{1}$ and $\mathbf{2}$ are likely preserved but are dynamic in solution at room temperature. Moreover, both $\mathbf{3}$ and $\mathbf{4}$ have dynamic solution structures where $\mathbf{3}$ is likely extensively dissociated in $\mathrm{CH}_{3} \mathrm{CN}$ or acetone rather than being polymeric as in the solid state.

\section{Introduction}

There is great interest in the development of coordination polymers because of their myriad possible uses in gas separation and storage, ${ }^{1}$ in optical ${ }^{2}$ and electronic materials, ${ }^{3}$ and even in biological/biomedical ${ }^{4}$ applications. Further interest is generated because the ability to modify the organic linker that bridges metal centers offers design opportunities to incorporate new functionality into a coordination polymer ${ }^{5}$ or to either probe fundamental or discover new principles of crystal engineering. ${ }^{6}$ Multitopic di(pyrazolyl)methane derivatives such as those in Chart $1^{7-11}$ and others ${ }^{12-16}$ have proven to be ideal candidates for such studies. Pioneering work by the Reger group on homoditopic $a, a, a^{\prime} a^{\prime}-$ tetra(pyrazol-1-yl)-( $p$ - or $m$-)xylene ligands, $p$ - or $m-\mathrm{pz}_{4} \mathrm{xyl}$ (Chart $1 \mathrm{~A}$, where $n=0$ ), showed that the reactions between $\mathrm{AgBF}_{4}$ and the homoditopic ligands in a 1:1 ratio resulted in a coordination polymer with $p-\mathrm{pz}_{4} \mathrm{xyl}$ but a cyclic bimetallic dication, $\left[\mathrm{Ag}_{2} \mathrm{~L}_{2}\right]^{2+}$, with $m-\mathrm{pz}_{4} \mathrm{xyl}{ }^{7 a}$ The three-dimensional assembly of the coordination polymer or the derivative with a cyclic dication was governed by ion pairing and directional noncovalent interactions such as the quadruple pyrazolyl embrace, ${ }^{12 \mathrm{~d}}$ a concerted set of $\mathrm{CH} \cdots \Pi$ and $\Pi \cdots \Pi$ interactions that has been found to be common in structures of metal complexes of poly (pyrazolyl)methane and borate ligands. For other metal complexes of $m-\mathrm{pz}_{4} \mathrm{xyl}$, the cyclic bimetallic supramolecular isomer is predominant, persists in solution, and allows for fundamental studies in electronic interactions between two metal centers. ${ }^{12}$ Our group recently examined the silver(I) trifluoromethanesulfonate complexes of the six isomers of homoditopic $a, a, a^{\prime} a^{\prime}$-tetra(pyrazol-1-yl)$(X, Y-)$ dimethylbiphenyl ligands $(X, Y=2,3$, or 4 , Chart $1 A, n=1, R=$ $\mathrm{H})$, which showed a similar isomer dependence; cyclic supramolecular isomers were obtained for two of the six possible isomers $(2,2-$ and 3,4-), whereas all other structurally characterized isomers were coordination polymers. ${ }^{8 a}$ Moreover $\mathrm{CH} \cdots \mathrm{O}$ interactions between triflate anion and the acidic methine and 5-pyrazolyl hydrogens dominated the 
supramolecular structures. Concurrently, the Manzano group showed $\mathrm{CH} \cdots X(X=O, F, C l, \pi)$ noncovalent interactions involving acidic methine, aryl, and pyrazolyl hydrogen and the pyrazolyl embrace govern the solid state assembly of coordination polymers and multimetallic complexes of related biphenyl-linked ligands (Chart1A, $n$ $=1, \mathrm{R}=\mathrm{Me}, 4,4$ - isomer). ${ }^{8 \mathrm{~b}}$ Heteroditopic ligands such as Manzano's (4-py) $\mathrm{CHpz}_{2}$ ( $\mathrm{pz} *=3,5$-dimethylpyrazol-1-yl, Chart $1 \mathrm{~B}$ where $\mathrm{R}=$ $\mathrm{Me}),{ }^{9}$ Carrano's (3- or 4- $\left.\mathrm{CO}_{2} \mathrm{HC}_{6} \mathrm{H}_{4}\right) \mathrm{CHpz}_{2}$ (Chart $1 \mathrm{C}, \mathrm{R}=\mathrm{Me}$ ), ${ }^{10}$ Marchio's (2- $\left.\mathrm{PhSC}_{6} \mathrm{H}_{4}\right) \mathrm{CHpz}_{2}{ }_{2}$ (Chart 1D), ${ }^{11}$ or others ${ }^{13-16}$ offer an attractive increase in complexity to the design of solid state architectures because of the different manners in which the ligands could bind metal centers. Thus, Figure 1a-f displays some of the different possible supramolecular isomers of $1: 1 \mathrm{M}: \mathrm{L}$ complexes of heteroditopic di(pyrazolyl)methane-based ligands that have been observed. The most common structure type is the cyclic bimetallic species in Figure 1d that has different donor ends of two ligands bound to a given metal. Similarly, the most common type of coordination polymer is found in Figure 1a or 1c (the difference being the orientation of the second donor group with respect to the aryl spacer). The complexes $\left.\left[\mathrm{Ag}\left(2-\mathrm{PhSC}_{6} \mathrm{H}_{4}\right) \mathrm{CHpz}_{2}\right]\right)\left(\mathrm{X}=\mathrm{BF}_{4}, \mathrm{PF}_{6}\right.$, or $\left.\mathrm{O}_{3} \mathrm{SCF}_{3}\right)$ showed an unusual hexameric metallacyclic structure (Figure 1f). The hexameric rings were inefficiently packed in the solid state by bridging anions and a host of other noncovalent interactions (similar to those shown in the bottom of Figure 1) to give permanent porosity to the crystalline solid and a remarkable capacity and selectivity for $\mathrm{CO}_{2}$ gas absorption. ${ }^{11}$

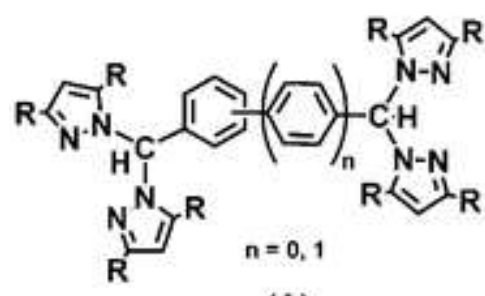

(A)

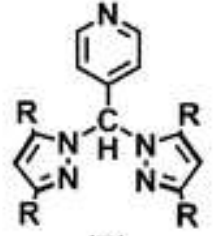

(B)

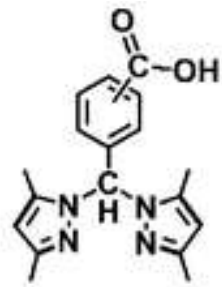

(C)

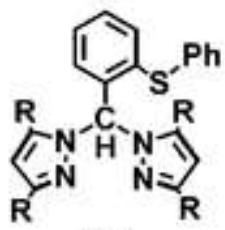

(D)

Chart 1. Representative Homo- and Hetero-Ditopic Aryldi(pyrazol-1yl)methane Ligands $(\mathrm{R}=\mathrm{H}, \mathrm{Me})$ 
polymeric

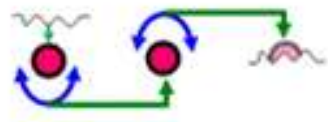

(a)

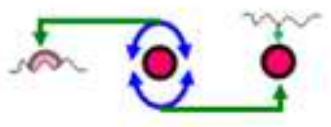

(b)

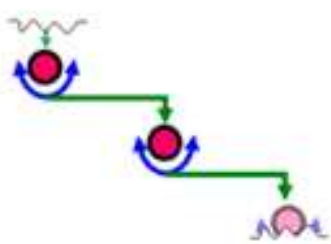

(c)

cyclic

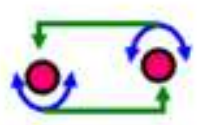

(d)

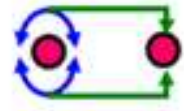

(e)

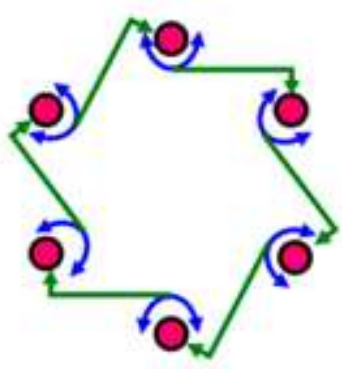

(f)

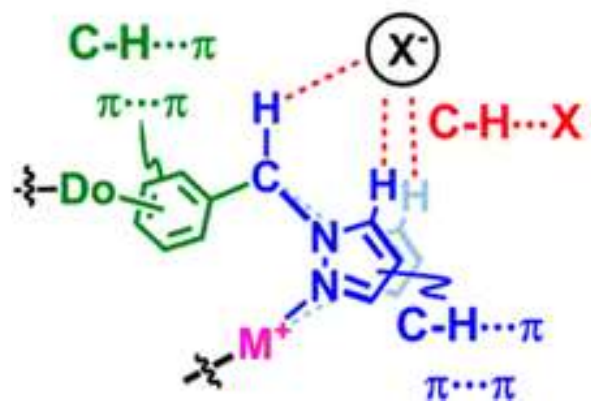

Figure 1. Representative supramolecular isomers of metal complexes of heteroditopic di(pyrazolyl)methane ligands (top and middle) and the common noncovalent interactions that organize their three-dimensional structures (bottom). Key: $\mathrm{Di}($ pyrazolyl)methane unit is blue, the other donor group (Do) and its attached aryl ring is green, and the metal center is pink.

Among the variety of heteroditopic di(pyrazolyl)methane type ligands that have been reported, we were surprised that those with diorganophosphines as a second donor remain unknown. Thus, we set out to prepare $\mathrm{R}_{2} \mathrm{PC}_{6} \mathrm{H}_{4} \mathrm{CHpz}_{2}$ derivatives and explore their coordination chemistry. This contribution outlines our initial endeavors in the preparation of such ligands, specifically, of the three isomers of ( $p$ tolyl $)_{2} \mathrm{P}\left(\mathrm{C}_{6} \mathrm{H}_{4}\right) \mathrm{CHpz}_{2}$. We also detail an investigation into their silver(I) trifluoromethanesulfonate complexes to learn more about the coordination capabilities of the ligands and to examine what effects, if any, a change in disposition of donors around a linking arene ring will 
have on supramolecular isomers and their three-dimensional crystal packing.

\section{Experimental Section}

\section{General Considerations}

$\mathrm{Pd}\left(\mathrm{PPh}_{3}\right)_{4}$ was prepared according to a literature procedure ${ }^{17}$ and stored under argon, and reactions employing this compound were performed under an argon atmosphere. $N, N^{\prime}$-Dimethylethylenediamine (DMED) and all other chemicals were commercially available and were used as received. Solvents were dried by conventional methods and distilled prior to use. $\mathrm{Di}(p$-tolyl)phosphine as a $10 \mathrm{wt} \%$ solution in hexanes was stored in an argon-filled drybox, and reactions employing this reagent were carried out with the exclusion of air by Schlenk techniques. The syntheses of the silver trifluoromethanesulfonate (AgOTf) complexes were carried out under an argon atmosphere using standard Schlenk techniques and in a foil-covered apparatus to protect AgOTf from light. After complex formation, no special precautions to avoid light or air were taken.

Midwest MicroLab, LLC, Indianapolis, Indiana 45250, performed all elemental analyses. IR spectra were recorded for samples as $\mathrm{KBr}$ pellets or as $\mathrm{CH}_{3} \mathrm{CN}$ solutions (solution cell with $\mathrm{KBr}$ windows) in the 4000-500 $\mathrm{cm}^{-1}$ region on a Nicolet Magna-IR 560 spectrometer. ${ }^{1} \mathrm{H}$, ${ }^{13} \mathrm{C},{ }^{19} \mathrm{~F}$, and ${ }^{31} \mathrm{P} \mathrm{NMR}$ spectra were recorded on a Varian $400 \mathrm{MHz}$ spectrometer. Chemical shifts were referenced to solvent resonances at $\delta_{H} 7.26$ and $\delta_{C} 77.16$ for $\mathrm{CDCl}_{3}, \delta_{H} 2.05$ for acetone- $d_{6}, \delta_{H} 1.94$ and $\delta_{\mathrm{C}} 118.26$ for $\mathrm{CD}_{3} \mathrm{CN}$ or against and external standards of $\mathrm{CFCl}_{3}\left(\delta_{\mathrm{F}}\right.$ $0.00 \mathrm{ppm}$ ) or of $85 \% \mathrm{H}_{3} \mathrm{PO}_{4}(\mathrm{aq})\left(\delta_{\mathrm{p}} 0.00 \mathrm{ppm}\right)$. The ${ }^{1} \mathrm{H}$ and ${ }^{13} \mathrm{C} \mathrm{NMR}$ data for 1-5 are labeled according to Figure S9, Supporting Information and 2D spectra are provided in Figures S10-S13, Supporting Information. Melting point determinations were made on samples contained in glass capillaries using an Electrothermal 9100 apparatus and are uncorrected. Mass spectrometric measurements recorded in $\mathrm{ESI}(+)$ mode were obtained on a Micromass Q-TOF spectrometer where formic acid (approximately $0.1 \% \mathrm{v} / \mathrm{v}$ ) was added to the mobile phase $\left(\mathrm{CH}_{3} \mathrm{CN}\right)$. 


\section{Syntheses}

\section{$\mathrm{p}-\mathrm{IC}_{6} \mathrm{H}_{4} \mathrm{CH}(\mathrm{pz})_{2}$}

A solution of $0.967 \mathrm{~g}(14.2 \mathrm{mmol})$ of pyrazole in $10 \mathrm{~mL}$ of THF was added to a suspension of $0.341 \mathrm{~g}(14.2 \mathrm{mmol})$ of $\mathrm{NaH}$ in $10 \mathrm{~mL}$ of THF via cannula at a rate slow enough to control hydrogen evolution The flask originally containing pyrazole was washed with an additional $5 \mathrm{~mL}$ of THF to ensure quantitative transfer. After hydrogen evolution ceased, a solution of $0.51 \mathrm{~mL}(1.638 \mathrm{~g} / \mathrm{mL}, 7.1 \mathrm{mmol})$ of $\mathrm{S}(0) \mathrm{Cl}_{2}$ in 10 $\mathrm{mL}$ of THF was added to the solution of $\mathrm{Na}(\mathrm{pz})$ whereupon a colorless precipitate of $\mathrm{NaCl}$ formed. After the suspension of $\mathrm{S}(\mathrm{O}) \mathrm{pz}_{2} / \mathrm{NaCl}$ had been stirred $30 \mathrm{~min}, 0.031 \mathrm{~g}(0.24 \mathrm{mmol})$ of $\mathrm{CoCl}_{2}$ was added as a solid in one portion under an argon blanket. After the resulting blue suspension had been stirred $5 \mathrm{~min}, 1.10 \mathrm{~g}(4.74 \mathrm{mmol})$ of $4-$ iodobenzaldehyde was added under an argon blanket. After the suspension had been heated at reflux or $15 \mathrm{~h}$, it was cooled to room temperature and $100 \mathrm{~mL}$ of water was added. The THF fraction was separated from the aqueous. Then the aqueous fraction was extracted with three $50 \mathrm{~mL}$ portions ethyl acetate. The combined organic fractions were dried over $\mathrm{MgSO}_{4}$ and filtered, and solvent was removed by rotary evaporation to leave $1.60 \mathrm{~g}(97 \%)$ of $p-\mathrm{IC}_{6} \mathrm{H}_{4} \mathrm{CH}(\mathrm{pz})_{2}$ as a white solid. Mp, $100-101{ }^{\circ} \mathrm{C} .{ }^{1} \mathrm{H}$ NMR $\left(400 \mathrm{MHz}, \mathrm{CDCl}_{3}\right) \delta_{\mathrm{H}} 7.70(\mathrm{~d}, \mathrm{~J}=$ $8.5 \mathrm{~Hz}, 2 \mathrm{H}, \mathrm{Ar}), 7.66\left(\mathrm{~s}, 1 \mathrm{H}, \mathrm{C}_{\text {meth }} \mathrm{H}\right), 7.63\left(\mathrm{~d}, J=1.5 \mathrm{~Hz}, 2 \mathrm{H}, \mathrm{H}_{3} \mathrm{pz}\right)$, $7.53\left(\mathrm{~d}, J=2.3 \mathrm{~Hz}, 2 \mathrm{H}, \mathrm{H}_{5} \mathrm{pz}\right), 6.75(\mathrm{~d}, J=8.5 \mathrm{~Hz}, 2 \mathrm{H}, \mathrm{Ar}), 6.35$ (pst, $\left.J_{\text {app }}=2 \mathrm{~Hz}, \mathrm{H}_{4} \mathrm{pz}\right) \mathrm{ppm} .{ }^{13} \mathrm{C}$ NMR $\left(100.5 \mathrm{MHz}, \mathrm{CDCl}_{3}\right) \delta_{\mathrm{c}} 141.1$, $138.0,136.1,129.9,128.9,107.0,95.6,77.4 \mathrm{ppm}$.

The following two compounds were prepared in a similar manner.

$m-\mathrm{IC}_{6} \mathrm{H}_{4} \mathrm{CH}(\mathrm{pz})_{2}$

A mixture of $5.8 \mathrm{mmol}$ of $\mathrm{S}(\mathrm{O}) \mathrm{pz}_{2}$ [from $0.279 \mathrm{~g}(11.6 \mathrm{mmol})$ of $\mathrm{NaH}, 0.792 \mathrm{~g}(11.6 \mathrm{mmol})$ of pyrazole, and $0.42 \mathrm{~mL}(1.638 \mathrm{~g} / \mathrm{mL}, 5.8$ $\mathrm{mmol})$ of $\left.\mathrm{S}(\mathrm{O}) \mathrm{Cl}_{2}\right], 0.025 \mathrm{~g}(0.19 \mathrm{mmol}) \mathrm{CoCl}_{2}$, and $0.900 \mathrm{~g}(3.88$ $\mathrm{mmol})$ of 3-iodobenzaldehyde gave $0.998 \mathrm{~g}(73 \%)$ of $m-\mathrm{IC}_{6} \mathrm{H}_{4} \mathrm{CH}(\mathrm{pz})_{2}$ as a colorless solid after column chromatography $\left(R_{f}=0.59,3: 1\right.$ hexanes: ethyl acetate, $\left.\mathrm{SiO}_{2}\right)$ and drying under a vacuum. Mp, 90$91^{\circ} \mathrm{C} .{ }^{1} \mathrm{H}$ NMR $\left(400 \mathrm{MHz}, \mathrm{CDCl}_{3}\right) \delta_{\mathrm{H}} 7.72(\mathrm{~d}, \mathrm{~J}=7.8 \mathrm{~Hz}, 1 \mathrm{H}, \mathrm{Ar}), 7.66$ 
$\left(\mathrm{s}, 1 \mathrm{H}, \mathrm{C}_{\text {meth }} \mathrm{H}\right), 7.64\left(\mathrm{~d}, J=1.6 \mathrm{~Hz}, 2 \mathrm{H}, \mathrm{H}_{3} \mathrm{pz}\right), 7.54(\mathrm{~d}, J=2.3 \mathrm{~Hz}, 2$ $\mathrm{H}, \mathrm{H}_{5} \mathrm{pz}$ ), 7.36 (s, $\left.1 \mathrm{H}, \mathrm{Ar}\right), 7.10$ (pst, Japp $=7.8 \mathrm{~Hz}, 1 \mathrm{H}, \mathrm{Ar}$ ), 6.98 (d, $\mathrm{J}=7.8 \mathrm{~Hz}, 1 \mathrm{H}, \mathrm{Ar}$ ), 6.36 (pst, $J_{\mathrm{app}}=2 \mathrm{~Hz}, 2 \mathrm{H}, \mathrm{H}_{4} \mathrm{pz}$ ) ppm. ${ }^{13} \mathrm{C} \mathrm{NMR}$ $\left(100.5 \mathrm{MHz}_{1} \mathrm{CDCl}_{3}\right) \delta_{\mathrm{C}} 141.2,138.6,138.5,136.0,130.6,129.9$, $126.4,107.1,94.7,76.9 \mathrm{ppm}$.

$\mathrm{O}-\mathrm{IC}_{6} \mathrm{H}_{4} \mathrm{CH}(\mathrm{pz})_{2}$

A mixture of $15.0 \mathrm{mmol}$ of $\mathrm{S}(\mathrm{O}) \mathrm{pz}_{2}$ [from $0.721 \mathrm{~g}(30.0 \mathrm{mmol})$ of $\mathrm{NaH}, 2.04 \mathrm{~g}(30.0 \mathrm{mmol})$ of pyrazole, and $1.09 \mathrm{~mL}(1.638 \mathrm{~g} / \mathrm{mL}$, $15.0 \mathrm{mmol})$ of $\left.\mathrm{S}(\mathrm{O}) \mathrm{Cl}_{2}\right], 0.130 \mathrm{~g}(1.00 \mathrm{mmol})$ of $\mathrm{CoCl}_{2}$, and $2.32 \mathrm{~g}$ $(10.0 \mathrm{mmol})$ of 2 -iodobenzaldehyde gave a yellow oily mixture after aqueous workup. The organic fraction was passed through a short (50 g) plug of silica gel with the aid of a 3:1 ( $v: v)$ ethyl acetate/hexane solution $\left(R_{f} 0.9\right)$ to separate from two pyrazolyl-containing impurities. After solvent was removed, the resulting pale yellow oil crystallized with the aid of scratching the glass to give $3.32 \mathrm{~g}(95 \%)$ of $\mathrm{o}$ $\mathrm{IC}_{6} \mathrm{H}_{4} \mathrm{CH}(\mathrm{pz})_{2}$ as a colorless solid. Mp, 81-82 ${ }^{\circ} \mathrm{C}$. ${ }^{1} \mathrm{H} \mathrm{NMR}(400 \mathrm{MHz}$, $\left.\mathrm{CDCl}_{3}\right) \delta_{\mathrm{H}} 7.90(\mathrm{dd}, J=7.9,1.1 \mathrm{~Hz}, 1 \mathrm{H}, \mathrm{Ar}), 7.80\left(\mathrm{~s}, 1 \mathrm{H}, \mathrm{C}_{\text {meth }} \mathrm{H}\right)$, 7.66 (dd, $J=1.8,0.5 \mathrm{~Hz}, 2 \mathrm{H}, \mathrm{H}_{3} \mathrm{pz}$ ), 7.35 (td, $J=7.3,1.2 \mathrm{~Hz}, 1 \mathrm{H}$, Ar), $7.32\left(\mathrm{~d}, \mathrm{~J}=2.5 \mathrm{~Hz}, 2 \mathrm{H}, \mathrm{H}_{5} \mathrm{pz}\right), 7.09(\mathrm{td}, \mathrm{J}=7.8,1.6 \mathrm{~Hz}, 1 \mathrm{H}$, Ar) , 6.73 (dd, J = 7.9, $1.5 \mathrm{~Hz}, 1 \mathrm{H}, \mathrm{Ar}$ ), 6.35 (pst, Japp $=1.9 \mathrm{~Hz}, 2 \mathrm{H}$, $\mathrm{H}_{4} \mathrm{pz}$ ) ppm. ${ }^{13} \mathrm{C} \mathrm{NMR}\left(100.5 \mathrm{MHz} \mathrm{CDCl}_{3}\right) \delta_{\mathrm{c}} 141.3,140.3,138.2$, $131.1,130.0,128.8,128.7,106.8,98.3,81.3$ ppm.

\section{$p$-(p-tolyl) ${ }_{2} \mathrm{PC}_{6} \mathrm{H}_{4} \mathrm{CH}(\mathrm{pz})_{2}, \boldsymbol{p L}$}

A solution of $1.009 \mathrm{~g}(2.88 \mathrm{mmol})$ of $p-\mathrm{IC}_{6} \mathrm{H}_{4} \mathrm{CH}(\mathrm{pz})_{2}, 0.10 \mathrm{~mL}$ $(0.82 \mathrm{~g} / \mathrm{mL}, 1.0 \mathrm{mmol})$ of DMED, and $20 \mathrm{~mL}$ of toluene was purged with argon 15 min and then was transferred via cannula to an argonpurged flask that contained $1.877 \mathrm{~g}(5.76 \mathrm{mmol})$ of $\mathrm{Cs}_{2} \mathrm{CO}_{3}, 0.017 \mathrm{~g}$ $(0.0015 \mathrm{mmol})$ of $\mathrm{Pd}\left(\mathrm{PPh}_{3}\right)_{4}$, and $10.0 \mathrm{~mL}(0.72 \mathrm{~g} / \mathrm{mL}, 10$ wt \% in hexanes, $3.36 \mathrm{mmol})$ of $\mathrm{HP}(p \text {-tolyl })_{2}$. After the resulting suspension was heated at reflux under argon $15 \mathrm{~h}$, toluene was removed by vacuum distillation to leave a black solid. The black solid was dissolved in a biphasic mixture of $100 \mathrm{~mL}$ of $\mathrm{H}_{2} \mathrm{O}$ and $50 \mathrm{~mL}$ of ethyl acetate. The layers were separated, and the aqueous portion was extracted with two $50 \mathrm{~mL}$ portions ethyl acetate. The combined organic fractions were dried over $\mathrm{MgSO}_{4}$ and filtered, and then solvent was removed by 
vacuum distillation. The resulting oily residue was purified by column chromatography in silica gel. Elution with 4:1 hexanes/ethyl acetate gave $0.987 \mathrm{~g}(79 \%)$ of $\boldsymbol{p L}$ as a viscous syrup after solvent was removed from the second band $\left(R_{f}=0.38\right) .{ }^{1} \mathrm{H} \mathrm{NMR}\left(400 \mathrm{MHz}, \mathrm{CDCl}_{3}\right)$ $\delta_{\mathrm{H}} 7.72\left(\mathrm{~s}, 1 \mathrm{H}, \mathrm{C}_{\text {meth }} \mathrm{H}\right), 7.62\left(\mathrm{~d}, J=1.3 \mathrm{~Hz}, 2 \mathrm{H}, \mathrm{H}_{3} \mathrm{pz}\right), 7.52(\mathrm{~d}, J=$ $\left.2.2 \mathrm{~Hz}, 2 \mathrm{H}, \mathrm{H}_{5} \mathrm{pz}\right), 7.25$ (d, $\left.J=7.5 \mathrm{~Hz}, 2 \mathrm{H}, \mathrm{Ar}\right), 7.18$ (d, J = $7.6 \mathrm{~Hz}, 4$ $\mathrm{H}, \mathrm{Ar}), 7.13(\mathrm{~d}, \mathrm{~J}=7.6 \mathrm{~Hz}, 4 \mathrm{H}, \mathrm{Ar}), 6.93(\mathrm{~d}, J=7.5 \mathrm{~Hz}, 2 \mathrm{H}, \mathrm{Ar})$, 6.32 (dd, $J=2,1 \mathrm{~Hz}, 2 \mathrm{H}, \mathrm{H}_{4} \mathrm{pz}$ ), $2.33\left(\mathrm{~s}, 6 \mathrm{H}, \mathrm{CH}_{3}\right) \mathrm{ppm} .{ }^{13} \mathrm{C} \mathrm{NMR}$ $\left(100.5 \mathrm{MHz}_{1} \mathrm{CDCl}_{3}\right) \delta_{\mathrm{C}} 140.8,140.0\left(\mathrm{~d}, J_{\mathrm{CP}}=12.8 \mathrm{~Hz}\right), 139.0,136.2$, $133.9\left(\mathrm{~d}, J_{C P}=20.1 \mathrm{~Hz}\right), 133.6\left(\mathrm{~d}, J_{\mathrm{CP}}=18.9 \mathrm{~Hz}\right), 133.1\left(\mathrm{~d}, J_{\mathrm{CP}}=9.0\right.$ $\mathrm{Hz}), 129.8,129.4\left(\mathrm{~d}, J_{\mathrm{CP}}=7.4 \mathrm{~Hz}\right), 126.9\left(\mathrm{~d}, J_{\mathrm{CP}}=6.4 \mathrm{~Hz}\right), 106.7$, 77.6, 21.3 ppm. ${ }^{31} \mathrm{P}$ NMR $\left(161.8 \mathrm{MHz} \mathrm{CDCl}_{3}\right) \delta_{\mathrm{P}}-7.6 \mathrm{ppm}$.

The following two compounds were prepared similarly.

\section{$m-(p-\text { tolyl })_{2} \mathrm{PC}_{6} \mathrm{H}_{4} \mathrm{CH}(p z)_{2}, \boldsymbol{m L}$}

A mixture of $0.931 \mathrm{~g}(2.86 \mathrm{mmol})$ of $\mathrm{Cs}_{2} \mathrm{CO}_{3}, 5.10 \mathrm{~mL}(0.72$ $\mathrm{g} / \mathrm{mL}, 10$ wt $\%$ in hexanes, $1.71 \mathrm{mmol})$ of $\mathrm{HP}(p$-tolyl $) 2,0.0083 \mathrm{~g}(7.2$ $\mu \mathrm{mol})$ of $\mathrm{Pd}\left(\mathrm{PPh}_{3}\right)_{4}, 0.500 \mathrm{~g}(1.43 \mathrm{mmol})$ of $m-\mathrm{IC}_{6} \mathrm{H}_{4} \mathrm{CH}(\mathrm{pz})_{2}, 0.10 \mathrm{~mL}$ $(0.82 \mathrm{~g} / \mathrm{mL}, 0.86 \mathrm{mmol})$ of DMED and $15 \mathrm{~mL}$ of toluene gave $0.229 \mathrm{~g}$ $(37 \%) \boldsymbol{m L}$ as a colorless oil after solvent was removed from the second band obtained from column chromatography $\left(R_{f}=0.69,3: 1\right.$ hexanes: ethyl acetate, $\left.\mathrm{SiO}_{2}\right) .{ }^{1} \mathrm{H} \mathrm{NMR}\left(400 \mathrm{MHz}, \mathrm{CDCl}_{3}\right) \delta_{\mathrm{H}} 7.65(\mathrm{~s}, 1$ $\mathrm{H}, \mathrm{C}_{\text {meth }} \mathrm{H}$ ), 7.55 (d, J = $\left.1.3 \mathrm{~Hz}, 2 \mathrm{H}, \mathrm{H}_{3} \mathrm{pz}\right), 7.42(\mathrm{~d}, J=2.4 \mathrm{~Hz}, 2 \mathrm{H}$, $\left.\mathrm{H}_{5} \mathrm{pz}\right), 7.29(\mathrm{~m}, J=7.6 \mathrm{~Hz}, 3 \mathrm{H}, \mathrm{Ar}), 7.10$ (br m, $\left.9 \mathrm{H}, \mathrm{Ar}\right), 6.91$ (d, J = $6.5 \mathrm{~Hz}, 1 \mathrm{H}, \mathrm{Ar}), 6.85$ (d, J = 7.2 Hz, $1 \mathrm{H}, \mathrm{Ar}), 6.25$ (dd, J = 2, $1 \mathrm{~Hz}, 2$ $\left.\mathrm{H}, \mathrm{H}_{4} \mathrm{pz}\right), 2.32\left(\mathrm{~s}, 6 \mathrm{H}, \mathrm{CH}_{3}\right) \mathrm{ppm} .{ }^{13} \mathrm{C} \mathrm{NMR}\left(100.5 \mathrm{MHz} \mathrm{CDCl}_{3}\right) \delta_{\mathrm{C}}$ $140.8,139.4\left(\mathrm{~d}, J_{\mathrm{CP}}=13.3 \mathrm{~Hz}\right), 139.0,136.4\left(\mathrm{~d}, J_{\mathrm{CP}}=6.2 \mathrm{~Hz}\right), 134.3$ $\left(d, J_{C P}=18.4 \mathrm{~Hz}\right), 133.8\left(d, J_{C P}=19.6 \mathrm{~Hz}\right), 133.1\left(\mathrm{~d}, J_{\mathrm{CP}}=9.5 \mathrm{~Hz}\right)$, $131.9\left(\mathrm{~d}, J_{\mathrm{CP}}=19.8 \mathrm{~Hz}\right), 129.7,129.5\left(\mathrm{~d}, J_{\mathrm{CP}}=7.3 \mathrm{~Hz}\right), 128.8\left(\mathrm{~d}, J_{\mathrm{CP}}\right.$ $=6.3 \mathrm{~Hz}), 129.9,106.6,77.7,21.4 \mathrm{ppm} .{ }^{31} \mathrm{P} \mathrm{NMR}\left(161.8 \mathrm{MHz}, \mathrm{CDCl}_{3}\right)$ $\delta_{\mathrm{p}}-7.1 \mathrm{ppm}$.

\section{$\mathrm{O}-(p-\text { tolyl })_{2} \mathrm{PC}_{6} \mathrm{H}_{4} \mathrm{CH}(\mathrm{pz})_{2}, \mathrm{oL}$}

A mixture of $0.972 \mathrm{~g}(2.98 \mathrm{mmol})$ of $\mathrm{Cs}_{2} \mathrm{CO}_{3}, 5.30 \mathrm{~mL}(0.72$ $\mathrm{g} / \mathrm{mL}, 10$ wt $\%$ soln in hexanes, $1.79 \mathrm{mmol})$ of $\mathrm{HP}(p \text {-tolyl })_{2}, 0.0086 \mathrm{~g}$ $(7.8 \mu \mathrm{mol})$ of $\mathrm{Pd}\left(\mathrm{PPh}_{3}\right)_{4}, 0.523 \mathrm{~g}(1.49 \mathrm{mmol})$ of $o-\mathrm{IC}_{6} \mathrm{H}_{4} \mathrm{CH}(\mathrm{pz})_{2}, 0.05$ 
$\mathrm{mL}(0.82 \mathrm{~g} / \mathrm{mL}, 0.48 \mathrm{mmol})$ of DMED and $15 \mathrm{~mL}$ of toluene gave 0.594 $\mathrm{g}(91 \%)$ oL as a colorless solid after solvent was removed from the second band obtained from column chromatography $\left(R_{f}=0.46,4: 1\right.$ hexanes: ethyl acetate, $\left.\mathrm{SiO}_{2}\right)$. Mp, $116-117^{\circ} \mathrm{C} .{ }^{1} \mathrm{H} \mathrm{NMR}(400 \mathrm{MHz}$, $\left.\mathrm{CDCl}_{3}\right) \delta_{\mathrm{H}} 8.55(\mathrm{~d}, \mathrm{~J}=7.6 \mathrm{~Hz}, 1 \mathrm{H}, \mathrm{Ar}), 7.49(\mathrm{~d}, J=1.3 \mathrm{~Hz}, 2 \mathrm{H}$, $\left.\mathrm{H}_{3} \mathrm{pz}\right), 7.36(\mathrm{t}, \mathrm{J}=7.3 \mathrm{~Hz}, 1 \mathrm{H}, \mathrm{Ar}), 7.29(\mathrm{t}, \mathrm{J}=7.3 \mathrm{~Hz}, 1 \mathrm{H}, \mathrm{Ar}), 7.18$ (d, J = 2.4 Hz, $2 \mathrm{H}, \mathrm{H}_{5} \mathrm{pz}$ ), 7.04 (br m, $10 \mathrm{H}, \mathrm{Ar}$ ), 6.10 (dd, J = 2, 1 $\left.\mathrm{Hz}, 2 \mathrm{H}, \mathrm{H}_{4} \mathrm{pz}\right), 2.31\left(\mathrm{~s}, 6 \mathrm{H}, \mathrm{CH}_{3}\right)$ ppm. ${ }^{13} \mathrm{C} \mathrm{NMR}\left(100.5 \mathrm{MHz} \mathrm{CDCl}_{3}\right)$ $\delta_{\mathrm{C}} 140.9,140.4\left(\mathrm{~d}, J_{\mathrm{CP}}=23.4 \mathrm{~Hz}\right), 138.8,137.0\left(\mathrm{~d}, J_{\mathrm{CP}}=18.5 \mathrm{~Hz}\right)$, $134.7,133.9\left(d, J_{C P}=19.9 \mathrm{~Hz}\right), 132.0\left(d, J_{C P}=6.9 \mathrm{~Hz}\right), 129.9,129.5$ $\left(d, J_{C P}=3.8 \mathrm{~Hz}\right), 129.3\left(\mathrm{~d}, J_{\mathrm{CP}}=7.0 \mathrm{~Hz}\right), 127.5\left(\mathrm{~d}, J_{\mathrm{CP}}=4.0 \mathrm{~Hz}\right)$, 106.2, 77.4, $75.5\left(\mathrm{~d}, \mathrm{~J}_{\mathrm{CP}}=28.0 \mathrm{~Hz}\right), 21.4 \mathrm{ppm} .{ }^{31} \mathrm{P} \mathrm{NMR}(161.8 \mathrm{MHz}$, $\left.\mathrm{CDCl}_{3}\right) \delta_{\mathrm{p}}-20.1 \mathrm{ppm}$.

\section{$[A g(\boldsymbol{p L})](O T f), \mathbf{1}$}

A solution of $0.241 \mathrm{~g}(0.553 \mathrm{mmol})$ of $\boldsymbol{p L}$ in $10 \mathrm{~mL}$ of THF was added via cannula to a solution of $0.142 \mathrm{~g}(0.553 \mathrm{mmol})$ of AgOTf in $10 \mathrm{~mL}$ of THF. The flask originally containing $\boldsymbol{p L}$ was washed with $5 \mathrm{~mL}$ of $\mathrm{THF}$, and the washings were transferred to the reaction mixture to ensure quantitative transfer. After the resulting solution had been stirred $4 \mathrm{~h}$, solvent was removed under a vacuum. The residue was washed with two $5 \mathrm{~mL}$ portions $\mathrm{Et}_{2} \mathrm{O}$ and was dried under a vacuum to leave $0.328 \mathrm{~g}(86 \%)$ of $\mathbf{1}$ as a colorless solid. Mp, $258-259^{\circ} \mathrm{C}$ dec Anal. Calcd (Found) for $\mathrm{C}_{28} \mathrm{H}_{25} \mathrm{~F}_{3} \mathrm{~N}_{4} \mathrm{O}_{3} \mathrm{PSAg}$ : C, 48.50 (48.47); $\mathrm{H}, 3.63$ (3.72); N, 8.08 (8.08). ${ }^{1} \mathrm{H}$ NMR (400 MHz, CD $\left.{ }_{3} \mathrm{CN}\right) \delta_{\mathrm{H}} 8.25$ (d, $J=2.5$ $\left.\mathrm{Hz}, 2 \mathrm{H}, \mathrm{H}_{5} \mathrm{pz}\right), 8.06\left(\mathrm{~s}, 1 \mathrm{H}, \mathrm{C}_{\text {meth }} \mathrm{H}\right), 7.84$ (d, J = $1.7 \mathrm{~Hz}, 2 \mathrm{H}, \mathrm{H}_{3} \mathrm{pz}$ ), 7.16 (dd, $J=11.5,8.1 \mathrm{~Hz}, 2 \mathrm{H}, \mathrm{H}_{2} \mathrm{Ar}$ ), 7.08 (dd, $J=8,2 \mathrm{~Hz}, 4 \mathrm{H}$, $\mathrm{H}_{3}$ Tol), 7.00 (dd, $J=11.7,7.0 \mathrm{~Hz}, 4 \mathrm{H}, \mathrm{H}_{2} \mathrm{Tol}$ ), 6.64 (d, $\mathrm{J}=8.0 \mathrm{~Hz}, 2$ $\mathrm{H}, \mathrm{H}_{3} \mathrm{Ar}$ ), 6.57 (dd, $\mathrm{J}=2,1 \mathrm{~Hz}, 2 \mathrm{H}, \mathrm{H}_{4} \mathrm{pz}$ ), $2.31\left(\mathrm{~s}, 6 \mathrm{H}, \mathrm{CH}_{3}\right) \mathrm{ppm}$. ${ }^{13} \mathrm{C}$ NMR $\left(100.5 \mathrm{MHz}, \mathrm{CD}_{3} \mathrm{CN}\right) \delta_{\mathrm{C}} 144.5\left(\mathrm{C}_{3} \mathrm{pz}\right), 142.2\left(\mathrm{C}_{4}{ }^{\mathrm{tol}}\right), 139.6$ $\left(\mathrm{C}_{4}{ }^{\mathrm{Ar}}\right), 135.5\left(\mathrm{~d}, \mathrm{~J}_{\mathrm{CP}}=18.4 \mathrm{~Hz}, \mathrm{C}_{2}{ }^{\mathrm{Ar}}\right), 135.0\left(\mathrm{C}_{5} \mathrm{pz}\right), 134.2\left(\mathrm{~d}, \mathrm{~J}_{\mathrm{CP}}=\right.$ $\left.16.8 \mathrm{~Hz}, \mathrm{C}_{2}{ }^{\text {tol }}\right), 133.2\left(\mathrm{~d}, \mathrm{~J}_{\mathrm{CP}}=35.8 \mathrm{~Hz}, \mathrm{C}_{1}\right.$ tol or $\left.\mathrm{Ar}\right), 130.6\left(\mathrm{~d}, \mathrm{~J}_{\mathrm{CP}}=10.3\right.$ $\left.\mathrm{Hz}, \mathrm{C}_{3}{ }^{\text {tol }}\right), 128.5\left(\mathrm{~d}, J_{\mathrm{CP}}=36.7 \mathrm{~Hz}, \mathrm{C}_{1}{ }^{\text {Ar or tol }}\right), 128.0\left(\mathrm{~d}, \mathrm{~J}_{\mathrm{CP}}=11.3 \mathrm{~Hz}\right.$, $\left.\mathrm{C}_{3}{ }^{\mathrm{Ar}}\right), 107.7\left(\mathrm{C}_{4} \mathrm{pz}\right), 75.2\left(\mathrm{C}_{\text {meth }}\right), 21.4\left(\mathrm{CH}_{3}\right)$ ppm. ${ }^{19} \mathrm{~F}$ NMR (376.1 $\left.\mathrm{MHz}, \mathrm{CD}_{3} \mathrm{CN}, 295 \mathrm{~K}\right) \delta_{\mathrm{F}}-79.3(\mathrm{~s}, 3 \mathrm{~F}) \mathrm{ppm} .{ }^{31} \mathrm{P} \mathrm{NMR}(161.8 \mathrm{MHz}$, $\left.\mathrm{CD}_{3} \mathrm{CN}\right) \delta_{\mathrm{p}} 11.3\left(\mathrm{~d}, J_{109 \mathrm{AgP}}=736 \mathrm{~Hz}, J_{107 \mathrm{AgP}}=638 \mathrm{~Hz}\right.$ ) ppm. LRMS [ESI(+), m/z] (Int.) [assign.]: 1417 (1) $\left[\mathrm{AgL}_{3}\right]^{+}, 1237$ (2) $\left[\mathrm{Ag}_{2} \mathrm{~L}_{2}(\mathrm{OTf})\right]^{+}, 981(12)\left[\mathrm{AgL}_{2}\right]^{+}, 762(39)\left[\mathrm{Ag}_{2} \mathrm{~L}_{3}\right]^{2+}, 544(100)$ 
$\left[\mathrm{Ag}_{2} \mathrm{~L}_{2}\right]^{2+}$. Crystals suitable for $\mathrm{X}$-ray diffraction were grown by layering an acetone solution of $\mathbf{1}$ with hexanes and allowing solvents to slowly diffuse over $20 \mathrm{~h}$.

\section{$[A g(\boldsymbol{m L})](O T f), \mathbf{2}$}

A solution of $0.161 \mathrm{~g}(0.368 \mathrm{mmol})$ of $\boldsymbol{m} \mathbf{L}$ in $10 \mathrm{~mL}$ of THF was transferred quantitatively (by washing the flask with additional $5 \mathrm{~mL}$ of THF and transferring the washings) via cannula to a solution of 0.095 $\mathrm{g}(0.368 \mathrm{mmol})$ of AgOTf in $10 \mathrm{~mL}$ of THF. A colorless precipitate formed immediately. After the suspension had been stirred $4 \mathrm{~h}$, the insoluble portion was collected by filtration, washed with two $5 \mathrm{~mL}$ portions $\mathrm{Et}_{2} \mathrm{O}$, and dried under a vacuum for $2 \mathrm{~h}$ to give $0.231 \mathrm{~g}(91 \%)$ of 2 as a colorless solid. Mp, $295-297^{\circ} \mathrm{C}$ dec Anal. Calcd (Found) for $\mathrm{C}_{28} \mathrm{H}_{25} \mathrm{~F}_{3} \mathrm{~N}_{4} \mathrm{O}_{3}$ PSAg: C, 48.50 (48.49); $\mathrm{H}, 3.63$ (3.75); N, 8.08 (7.90). ${ }^{1} \mathrm{H}$ NMR $\left(400 \mathrm{MHz}, \mathrm{CD}_{3} \mathrm{CN}\right) \delta_{\mathrm{H}} 8.13\left(\mathrm{~d}, \mathrm{~J}=2.4 \mathrm{~Hz}, 2 \mathrm{H}, \mathrm{H}_{5} \mathrm{pz}\right.$ ), 7.93 (s, $\left.1 \mathrm{H}, \mathrm{C}_{\text {meth }} \mathrm{H}\right), 7.67\left(\mathrm{~d}, \mathrm{~J}=1.4 \mathrm{~Hz}, 2 \mathrm{H}, \mathrm{H}_{5} \mathrm{pz}\right), 7.63$ (td, $\mathrm{J}=7.9,2.4 \mathrm{~Hz}$, $1 \mathrm{H}, \mathrm{C}_{5}{ }^{\mathrm{Ar}}$ ), 7.49 (dd, $J=12.9,7.4 \mathrm{~Hz}, 1 \mathrm{H}, \mathrm{C}_{6}{ }^{\mathrm{Ar}}$ ), 7.25 (d, J = $7.1 \mathrm{~Hz}, 4$ $\left.\mathrm{H}_{1} \mathrm{H}_{3}{ }^{\mathrm{tol}}\right), 6.89$ (dd, $\left.\mathrm{J}=12.2,8.0 \mathrm{~Hz}, 4 \mathrm{H}, \mathrm{H}_{2}{ }^{\mathrm{tol}}\right), 6.74(\mathrm{~d}, J=8 \mathrm{~Hz}, 1 \mathrm{H}$, $\mathrm{H}_{4}{ }^{\mathrm{Ar}}$ ), 6.43 (dd, $\mathrm{J}=2,1 \mathrm{~Hz}, 2 \mathrm{H}, \mathrm{H}_{4} \mathrm{pz}$ ), 5.61 (d, J = 9.5 Hz, $1 \mathrm{H}, \mathrm{C}_{2}{ }^{\mathrm{Ar}}$ ), $2.40\left(\mathrm{~s}, 6 \mathrm{H}, \mathrm{CH}_{3}\right) \mathrm{ppm} .{ }^{13} \mathrm{C}$ NMR (100.5 MHz, CD $\left.{ }_{3} \mathrm{CN}\right) \delta_{\mathrm{c}} 144.6\left(\mathrm{C}_{5} \mathrm{pz}\right)$, $143.2\left(\mathrm{C}_{4}{ }^{\mathrm{tol}}\right), 137.5\left(\mathrm{~d}, \mathrm{~J}_{\mathrm{CP}}=7.0 \mathrm{~Hz}, \mathrm{C}_{3}{ }^{\mathrm{Ar}}\right), 135.6\left(\mathrm{~d}, \mathrm{~J}_{\mathrm{CP}}=25.4 \mathrm{~Hz}\right.$, $\mathrm{C}_{1}{ }^{\mathrm{Ar} \text { or tol }}$ ), 135.0 (d, $\left.J_{\mathrm{CP}}=27 \mathrm{~Hz} \mathrm{C}_{6}{ }^{\mathrm{Ar}}\right), 134.7\left(\mathrm{C}_{5} \mathrm{pz}\right), 134.3\left(\mathrm{~d}, \mathrm{~J}_{\mathrm{CP}}=\right.$ $\left.34.6 \mathrm{~Hz}, \mathrm{C}_{2}{ }^{\mathrm{tol}}\right), 131.1\left(\mathrm{~d}, \mathrm{~J}_{\mathrm{CP}}=10.7 \mathrm{~Hz}, \mathrm{C}_{2}{ }^{\mathrm{Ar}}\right), 130.9\left(\mathrm{C}_{5} \mathrm{Ar}\right), 130.5$ $\left(\mathrm{C}_{3}{ }^{\mathrm{tol}}\right), 129.6\left(\mathrm{C}_{4}{ }^{\mathrm{Ar}}\right), 127.2\left(\mathrm{~d}, J_{\mathrm{CP}}=38.9 \mathrm{~Hz}, \mathrm{C}_{1}{ }^{\text {tol or } \mathrm{Ar}}\right), 107.3\left(\mathrm{C}_{4} \mathrm{pz}\right)$, 75.4 ( $\left.\mathrm{C}_{\text {meth }}\right), 21.3\left(\mathrm{CH}_{3}\right)$ ppm. ${ }^{19} \mathrm{~F}$ NMR (376.1 MHz, CD $\left.{ }_{3} \mathrm{CN}, 295 \mathrm{~K}\right) \delta_{\mathrm{F}}$ -79.3 (s, 3F) ppm. ${ }^{31} \mathrm{P}$ NMR (161.8 MHz, CD $\left.{ }_{3} \mathrm{CN}\right) \delta_{\mathrm{p}} 11.1$ (d, J109AgP $=$ $\left.740 \mathrm{~Hz} ; J_{107 \mathrm{AgP}}=644 \mathrm{~Hz}\right) \mathrm{ppm}$. LRMS [ESI(+), $\mathrm{m} / \mathrm{z}$ ] (Int.) [assign.]: 1417 (1) $\left[\mathrm{AgL}_{3}\right]^{+}, 1237$ (3) $\left[\mathrm{Ag}_{2} \mathrm{~L}_{2}(\mathrm{OTf})\right]^{+}, 981(21)\left[\mathrm{AgL}_{2}\right]^{+}, 762$ (25) $\left[\mathrm{Ag}_{2} \mathrm{~L}_{3}\right]^{2+}, 544(100)\left[\mathrm{Ag}_{2} \mathrm{~L}_{2}\right]^{2+}$. Crystals suitable for $\mathrm{X}$-ray diffraction were grown over the course of $12 \mathrm{~h}$ by vapor diffusion of $\mathrm{Et}_{2} \mathrm{O}$ into an acetonitrile solution of $\mathbf{2}$.

\section{$[\mathrm{Ag}(\mathrm{oL})](\mathrm{OTf}), \mathbf{3}$}

A solution of $0.178 \mathrm{~g}(0.407 \mathrm{mmol})$ of oL in $10 \mathrm{~mL}$ of THF was transferred quantitatively (by washing the flask with additional $5 \mathrm{~mL}$ of THF and transferring the washings) via cannula to a solution of 0.105 $\mathrm{g}(0.407 \mathrm{mmol})$ of AgOTf in $10 \mathrm{~mL}$ of THF. A colorless precipitate 
formed immediately. After the suspension had been stirred $4 \mathrm{~h}$, the insoluble portion was collected by filtration, washed with two $5 \mathrm{~mL}$ portions $\mathrm{Et}_{2} \mathrm{O}$, and dried under a vacuum for $2 \mathrm{~h}$ to give $0.238 \mathrm{~g} \mathrm{(84 \% )}$ of 3 as a colorless solid. Mp, 225-227 ${ }^{\circ} \mathrm{C}$, dec Anal. Calcd (Found) for $\mathrm{C}_{28} \mathrm{H}_{25} \mathrm{~F}_{3} \mathrm{~N}_{4} \mathrm{O}_{3}$ PSAg: C, 48.50 (48.59); $\mathrm{H}, 3.63$ (3.55); N, 8.08 (7.96). ${ }^{1} \mathrm{H}$ NMR (400 MHz, CD $\left.{ }_{3} \mathrm{CN}, 295 \mathrm{~K}\right) \delta_{\mathrm{H}} 8.35\left(\mathrm{~d}, J=5.0 \mathrm{~Hz}, 1 \mathrm{H}, \mathrm{C}_{\text {meth }} \mathrm{H}\right)$, 7.84 (dd, $J=7.8,4.1 \mathrm{~Hz}, 1 \mathrm{H}, \mathrm{H}_{3}{ }^{\mathrm{Ar}}$ ), $7.58\left(\mathrm{t}, J=7.6 \mathrm{~Hz}, 1 \mathrm{H}, \mathrm{H}_{4}{ }^{\mathrm{Ar}}\right.$ ), 7.44-7.42 (br m, $3 \mathrm{H}, \mathrm{H}_{5} \mathrm{Ar}$ and $\mathrm{H}_{5} \mathrm{pz}$ ), 7.30 (d, $J=2 \mathrm{~Hz}, 2 \mathrm{H}, \mathrm{H}_{3} \mathrm{pz}$ ) 7.27 (br m, $4 \mathrm{H}_{,} \mathrm{H}_{2}{ }^{\mathrm{tol}}$ ), 7.26 (br m, $4 \mathrm{H}_{,} \mathrm{H}_{3}{ }^{\mathrm{tol}}$ ), 6.93 (t, $\mathrm{J}=8.3 \mathrm{~Hz}, 1 \mathrm{H}$, $\mathrm{H}_{6}{ }^{\mathrm{Ar}}$ ), 6.21 (dd, $J=2,1 \mathrm{~Hz}, 2 \mathrm{H}, \mathrm{H}_{4} \mathrm{pz}$ ), $2.37\left(\mathrm{~s}, 6 \mathrm{H}, \mathrm{CH}_{3}\right) \mathrm{ppm} .{ }^{13} \mathrm{C}$ $\operatorname{NMR}\left(100.5 \mathrm{MHz}, \mathrm{CD}_{3} \mathrm{CN}, 295 \mathrm{~K}\right) \delta_{\mathrm{C}} 143.4\left(\mathrm{C}_{3} \mathrm{pz}\right), 142.2\left(\mathrm{C}_{4}{ }^{\mathrm{tol}}\right), 139.4$ $\left(\mathrm{d}, J_{C P}=14.1 \mathrm{~Hz}, \mathrm{C}_{2}{ }^{\mathrm{Ar}}\right), 135.3\left(\mathrm{~d}, J_{C P}=17.7 \mathrm{~Hz}, \mathrm{C}_{2}{ }^{\mathrm{tol}}\right), 135.1\left(\mathrm{C}_{5} \mathrm{pz}\right)$, $134.1\left(\mathrm{~d}, J_{\mathrm{CP}}=2.7 \mathrm{~Hz}, \mathrm{C}_{6}{ }^{\mathrm{Ar}}\right), 132.3\left(\mathrm{C}_{4}{ }^{\mathrm{Ar}}\right), 132.1\left(\mathrm{~d}, \mathrm{~J}_{\mathrm{CP}}=26 \mathrm{~Hz}\right.$, $\left.\mathrm{C}_{1}{ }^{\text {Ar or tol }}\right), 131.4\left(\mathrm{C}_{3}{ }^{\mathrm{tol}}\right), 131.3\left(\mathrm{~d}, \mathrm{~J}_{C P}=4.8 \mathrm{~Hz}, \mathrm{C}_{5}{ }^{\mathrm{Ar}}\right), 130.6\left(\mathrm{~d}, J_{\mathrm{CP}}=6.0\right.$ $\left.\mathrm{Hz}, \mathrm{C}_{3}{ }^{\mathrm{Ar}}\right), 126.6\left(\mathrm{~d}, \mathrm{~J}_{\mathrm{CP}}=34.7 \mathrm{~Hz}, \mathrm{C}_{1}\right.$ tol or Ar$), 107.8\left(\mathrm{C}_{4} \mathrm{pz}\right), 75.0\left(\mathrm{~d}, \mathrm{~J}_{C P}\right.$ $\left.=20.2 \mathrm{~Hz}, \mathrm{C}_{\text {meth }}\right), 21.4 \mathrm{ppm} .{ }^{19} \mathrm{~F} \mathrm{NMR}\left(376.1 \mathrm{MHz}, \mathrm{CD}_{3} \mathrm{CN}, 295 \mathrm{~K}\right) \delta_{\mathrm{F}}$ -79.3 (s, 3F) ppm. ${ }^{31} \mathrm{P}$ NMR (161.8 MHz, $\mathrm{CD}_{3} \mathrm{CN}, 295 \mathrm{~K}$ ) $\delta_{\mathrm{P}}-1.1$ (br s, $\mathrm{LWHM}=245 \mathrm{~Hz}) \mathrm{ppm} ;(233 \mathrm{~K}) \delta_{\mathrm{p}}-1.6\left(\mathrm{~d}, J_{109 \mathrm{AgP}}=660 \mathrm{~Hz} ; J_{107 \mathrm{AgP}}=\right.$ $582 \mathrm{~Hz}$ ) ppm. LRMS [ESI(+), $m / z, \mathrm{CH}_{3} \mathrm{CN} / \mathrm{MeOH}$ (Int.) [assign.]: 1703 (8) $\left[\mathrm{Ag}_{2} \mathrm{~L}_{3}(\mathrm{OTf})(\mathrm{MeOH})\right]^{+}, 1237$ (2) $\left[\mathrm{Ag}_{2} \mathrm{~L}_{2}(\mathrm{OTf})\right]^{+}, 1123$ (10) $\left[\mathrm{Ag}_{3} \mathrm{~L}_{4}(\mathrm{OTf})(\mathrm{MeOH})\right]^{2+}, 979(100)\left[\mathrm{AgL}_{2}\right]^{+}, 687$ (21) $\left[\mathrm{Ag}_{3} \mathrm{~L}_{2}(\mathrm{OTf})(\mathrm{MeOH})\right]^{2+}, 584(25)\left[\mathrm{AgL}\left(\mathrm{CH}_{3} \mathrm{CN}\right)\right]^{+}, 543$ (5) $[\mathrm{AgL}]^{+}$. Crystals suitable for X-ray diffraction were grown over the course of 12 $\mathrm{h}$ by vapor diffusion of $\mathrm{Et}_{2} \mathrm{O}$ into an acetonitrile solution of $\mathbf{3}$.

\section{$\left[A g(o L)\left(P P h_{3}\right)\right](O T f), 4$}

Upon addition of $0.0265 \mathrm{~g}(0.101 \mathrm{mmol})$ of $\mathrm{PPh}_{3}$ as a solid to a stirred suspension of $0.0700 \mathrm{~g}(0.101 \mathrm{mmol})$ of 3 in $20 \mathrm{~mL}$ of $\mathrm{CH}_{2} \mathrm{Cl}_{2}$, a colorless solution formed. After the solution had been stirred $1 \mathrm{~h}$ at room temperature, solvent was removed under a vacuum. The residue was washed with two $5 \mathrm{~mL}$ portions hexane and was dried under a vacuum to leave $0.0835 \mathrm{~g}(87 \%) 4$ as a colorless solid. Mp, 225$226{ }^{\circ} \mathrm{C}$, dec Anal. Calcd (Found) for $\mathrm{C}_{46} \mathrm{H}_{40} \mathrm{~F}_{3} \mathrm{~N}_{4} \mathrm{O}_{3} \mathrm{P}_{2} \mathrm{SAg}$ : C, 57.81 (57.68); H, 4.22 (4.18); N, 5.86 (5.84). ${ }^{1} \mathrm{H}$ NMR (400 MHz, acetone$\left.\mathrm{d}_{6}, 295 \mathrm{~K}\right) \delta_{\mathrm{H}} 8.75(\mathrm{br} \mathrm{s}, 1 \mathrm{H}), 8.07$ (br d, $\left.\mathrm{J}=8 \mathrm{~Hz}, 1 \mathrm{H}\right), 7.71-7.43$ (br m, $20 \mathrm{H}$ ), 7.41-7.21 (br m, $9 \mathrm{H}$ ), 7.06 (br m, $1 \mathrm{H}), 6.24$ (br s, $2 \mathrm{H}$, $\mathrm{H}_{4} \mathrm{pz}$ ), 2.38 (br s, $\left.6 \mathrm{H}, \mathrm{CH}_{3}\right) \mathrm{ppm} .{ }^{13} \mathrm{C} \mathrm{NMR}\left(100.5 \mathrm{MHz}\right.$, acetone- $d_{6}$, $295 \mathrm{~K}) \delta_{c}$ not reported because broad resonances and low signal-to- 
noise gave uninformative spectrum even after reasonable acquisition period of $15 \mathrm{~h} .{ }^{19} \mathrm{~F} \mathrm{NMR}\left(376.1 \mathrm{MHz}\right.$, acetone- $\left.d_{6}, 295 \mathrm{~K}\right) \delta_{\mathrm{F}}-79.1$ (s, 3F) ppm. ${ }^{31} \mathrm{P}$ NMR $\left(161.8 \mathrm{MHz}\right.$, acetone- $\left.d_{6}, 295 \mathrm{~K}\right) \delta_{\mathrm{p}}$ br $\mathrm{s}+11.8$ $\left(\mathrm{LWHM}=290 \mathrm{~Hz}, 1 \mathrm{P}, \mathrm{PPh}_{3}\right)$, br s $-2.1(\mathrm{LWHM}=599 \mathrm{~Hz}, 1 \mathrm{P}, \mathrm{P}(p-$ tolyl)2) ppm; (193 K) $\delta_{\mathrm{P}} 11.6\left(\mathrm{dd}, J_{109 \mathrm{Ag}-\mathrm{P}}=558 \mathrm{~Hz}, J_{107 \mathrm{Ag}-\mathrm{P}}=484 \mathrm{~Hz}\right.$, $\left.J_{\mathrm{P}-\mathrm{P}}=108 \mathrm{~Hz}, 1 \mathrm{P}, \mathrm{PPh}_{3}\right),-2.4\left(\mathrm{dd}, J_{109 \mathrm{Ag}-\mathrm{P}}=457 \mathrm{~Hz}, J_{107 \mathrm{Ag}-\mathrm{P}}=396 \mathrm{~Hz}\right.$, $\left.J_{\mathrm{P}-\mathrm{P}}=108 \mathrm{~Hz}, 1 \mathrm{P}, \mathrm{P}(p \text {-tolyl })_{2}\right) \mathrm{ppm}$. Crystals suitable for X-ray diffraction were grown over the course of $15 \mathrm{~h}$ by allowing a layer of hexane to diffuse into a dichloromethane solution of 4 .

\section{$\left[\mathrm{Ag}\left(\mathrm{m}-\mathrm{IC}_{6} \mathrm{H}_{4} \mathrm{CH}(\mathrm{pz})_{2}\right)_{2}\right](\mathrm{OTf}), \mathbf{5}$}

A solution of $0.304 \mathrm{~g}(0.868 \mathrm{mmol})$ of $m-\mathrm{IC}_{6} \mathrm{H}_{4} \mathrm{CH}(\mathrm{pz})_{2}$ in $10 \mathrm{~mL}$ of THF was transferred quantitatively (by washing the flask with an additional $5 \mathrm{~mL}$ of THF and transferring the washings) via cannula to a solution of $0.112 \mathrm{~g}(0.434 \mathrm{mmol})$ of AgOTf in $5 \mathrm{~mL}$ of THF. A colorless precipitate formed after several hours. After the mixture had been stirred $12 \mathrm{~h}$, the insoluble portion was collected by filtration, washed with two $5 \mathrm{~mL}$ portions $\mathrm{Et}_{2} \mathrm{O}$, and dried under a vacuum for $2 \mathrm{~h}$ to give $0.356 \mathrm{~g}(86 \%)$ of 5 as a colorless solid. Mp, $220-221^{\circ} \mathrm{C}$ dec Anal. Calcd (Found) for $\mathrm{C}_{27} \mathrm{H}_{22} \mathrm{~F}_{3} \mathrm{I}_{2} \mathrm{~N}_{8} \mathrm{O}_{3} \mathrm{SAg}: \mathrm{C}, 33.88$ (33.73); $\mathrm{H}, 2.32$ (2.44); $\mathrm{N}, 11.71$ (11.64). ${ }^{1} \mathrm{H}$ NMR (400 MHz, $\left.\mathrm{CD}_{3} \mathrm{CN}\right) \delta_{\mathrm{H}} 7.85$ (d, $J=$ $\left.2.5 \mathrm{~Hz}, 2 \mathrm{H}, \mathrm{H}_{5} \mathrm{pz}\right), 7.82\left(\mathrm{~s}, 1 \mathrm{H}, \mathrm{C}_{\text {meth }} \mathrm{H}\right), 7.78(\mathrm{~d}, \mathrm{~J}=7.9 \mathrm{~Hz}, 1 \mathrm{H}$, $\left.\mathrm{C}_{6}{ }^{\mathrm{Ar}}\right), 7.59\left(\mathrm{~d}, \mathrm{~J}=1.8 \mathrm{~Hz}, 2 \mathrm{H}, \mathrm{H}_{3} \mathrm{pz}\right), 7.20\left(\mathrm{~s}, 1 \mathrm{H}, \mathrm{H}_{2}{ }^{\mathrm{Ar}}\right), 7.16(\mathrm{t}, J=$ $\left.7.9 \mathrm{~Hz}, 1 \mathrm{H}, \mathrm{H}_{5}{ }^{\mathrm{Ar}}\right), 6.88\left(\mathrm{~d}, \mathrm{~J}=7.9 \mathrm{~Hz}, 1 \mathrm{H}, \mathrm{H}_{4}{ }^{\mathrm{Ar}}\right), 6.42$ (dd, $\mathrm{J}=3,2$ $\mathrm{Hz}, 2 \mathrm{H}, \mathrm{H}_{4} \mathrm{pz}$ ) ppm. ${ }^{13} \mathrm{C} \mathrm{NMR}\left(100.5 \mathrm{MHz}, \mathrm{CD}_{3} \mathrm{CN}\right) \delta_{\mathrm{c}} 142.7$ ( $\left.\mathrm{C}_{3} \mathrm{pz}\right)$, $139.4\left(\mathrm{C}_{6}{ }^{\mathrm{Ar}}\right), 139.3\left(\mathrm{C}_{3}{ }^{\mathrm{Ar}}\right), 136.6\left(\mathrm{C}_{2}{ }^{\mathrm{Ar}}\right), 132.8\left(\mathrm{C}_{5} \mathrm{pz}\right), 131.5\left(\mathrm{C}_{5}{ }^{\mathrm{Ar}}\right)$, $127.4\left(\mathrm{C}_{4}{ }^{\mathrm{Ar}}\right), 107.5\left(\mathrm{C}_{4} \mathrm{pz}\right), 94.6\left(\mathrm{C}_{1}{ }^{\mathrm{Ar}}\right), 76.0$ ( $\left.\mathrm{C}_{\text {meth }}\right)$ ppm. LRMS [ESI(+), m/z] (Int.) [assign.]: 807 (100) $\left[\mathrm{AgL}_{2}\right]^{+}, 498$ (23) $\left[\mathrm{AgL}\left(\mathrm{CH}_{3} \mathrm{CN}\right)\right]+, 351(50)[\mathrm{HL}]^{+}, 283$ (92) [L-pz]. Crystals suitable for $\mathrm{X}$-ray diffraction were grown over the course of $12 \mathrm{~h}$ by vapor diffusion of $\mathrm{Et}_{2} \mathrm{O}$ into an acetonitrile solution of $\mathbf{5}$.

\section{Crystallography}

X-ray intensity data from a colorless block of $\mathbf{1}$-acetone, a colorless prism of $\mathbf{2} \cdot \mathrm{CH}_{3} \mathrm{CN}$, a colorless needle of $\mathbf{3}$, a colorless prism of 4, and a colorless prism of $\mathbf{5}$ were collected at 100.0(1) K with an Oxford Diffraction Ltd. Supernova diffractometer equipped with a 135 
$\mathrm{mm}$ Atlas CCD detector using $\mathrm{Mo}(\mathrm{Ka})$ radiation for $\mathbf{1} \cdot$ acetone, 2. $\mathrm{CH}_{3} \mathrm{CN}, \mathbf{4}$, and 5 but $\mathrm{Cu}(\mathrm{Ka})$ for 3. Raw data frame integration and Lp corrections were performed with CrysAlis Pro (Oxford Diffraction, Ltd.). ${ }^{18}$ Final unit cell parameters were determined by least-squares refinement of $34071,14148,14020,22446$, and 15970 reflections of 1.acetone, $\mathbf{2} \cdot \mathrm{CH}_{3} \mathrm{CN}, \mathbf{3}, \mathbf{4}$, and 5, respectively, with $I>2 \sigma(I)$ for each. Analysis of the data showed negligible crystal decay during collection in each case. Direct methods structure solutions, difference Fourier calculations, and full-matrix least-squares refinements against $\mathrm{F}^{2}$ were performed with Olex ${ }^{19}$ and SHELXTL. ${ }^{20} \mathrm{~A}$ numerical absorption correction based on Gaussian integration over a multifaceted crystal model were applied to the data for each crystal. All non-hydrogen atoms were refined with anisotropic displacement parameters. Hydrogen atoms were placed in geometrically idealized positions and included as riding atoms. For $\mathbf{3}$, the unit cell contains four large void spaces with volumes of approximately $266 A^{3}$ (1064/6487 or $16.4 \%$ of total cell volume). The voids are filled with heavily disordered solvent (ca. 1:1 $\mathrm{Et}_{2} \mathrm{O} / \mathrm{CH}_{3} \mathrm{CN}$ ). The solvent mask routine implemented in Olex2 was applied to the data to account for the electron density from these solvents. In the structure of $\mathbf{4}$, the pyrazolyl group that is not bound to silver is rotationally disordered over two positions in a $1: 1$ ratio. The triflate anion is disordered over two positions in an 88:12 ratio. The $\mathrm{X}-$ ray crystallographic parameters and further details of data collection and structure refinements are given in Table 1.

Table 1. Crystallographic Data Collection and Structure Refinement for 1·0.5 acetone, $\mathbf{2} \cdot \mathrm{CH}_{3} \mathrm{CN}, \mathbf{3}$, and $\mathbf{5}$

\begin{tabular}{|c|c|c|c|c|c|}
\hline compound & 1.0.5 acetone & $2 \cdot \mathrm{CH}_{3} \mathrm{CN}$ & 3 & 4 & 5 \\
\hline formula & $\begin{array}{l}\mathrm{C}_{59} \mathrm{H}_{56} \mathrm{Ag}_{2} \mathrm{~F}_{6} \mathrm{~N}_{8} \mathrm{O}_{7} \\
\mathrm{P}_{2} \mathrm{~S}_{2}\end{array}$ & $\begin{array}{l}\mathrm{C}_{60} \mathrm{H}_{56} \mathrm{Ag}_{2} \mathrm{~F}_{6} \mathrm{~N}_{10} \mathrm{O}_{6} \\
\mathrm{P}_{2} \mathrm{~S}_{2}\end{array}$ & $\begin{array}{l}\mathrm{C}_{28} \mathrm{H}_{25} \mathrm{AgF}_{3} \mathrm{~N}_{4} \mathrm{O} \\
{ }_{3} \mathrm{PS}\end{array}$ & $\begin{array}{l}\mathrm{C}_{46} \mathrm{H}_{40} \mathrm{AgF}_{3} \mathrm{~N}_{4} \mathrm{O}_{3} \\
\mathrm{P}_{2} \mathrm{~S}\end{array}$ & $\begin{array}{l}\mathrm{C}_{27} \mathrm{H}_{22} \mathrm{AgF}_{3} \mathrm{I}_{2} \mathrm{~N}_{8} \\
\mathrm{O}_{3} \mathrm{~S}\end{array}$ \\
\hline $\begin{array}{l}\text { formula } \\
\text { weight }\end{array}$ & 1444.92 & 1468.95 & 693.42 & 955.69 & 957.26 \\
\hline $\begin{array}{l}\text { crystal } \\
\text { system }\end{array}$ & monoclinic & monoclinic & orthorhombic & monoclinic & triclinic \\
\hline space group & $P 2_{1} / C$ & $P 2_{1} / n$ & Pbca & $P 2_{1} / n$ & $P 1^{-}$ \\
\hline $\begin{array}{l}\text { temperature [ } \\
\mathrm{K}]\end{array}$ & $100.0(1)$ & $100.0(1)$ & $100.0(1)$ & $100.0(1)$ & $100.0(1)$ \\
\hline$a[\AA]$ & $22.6768(3)$ & $11.8539(2)$ & $21.8299(3)$ & $9.34515(15)$ & $9.4676(3)$ \\
\hline$b[\AA]$ & $11.79225(13)$ & $15.5905(3)$ & $13.12475(11)$ & $13.8176(3)$ & $11.6230(3)$ \\
\hline$c[\AA]$ & $23.7381(3)$ & $17.6390(4)$ & $22.6416(3)$ & $33.1325(6)$ & $15.5967(4)$ \\
\hline $\mathrm{a}\left[^{\circ}\right]$ & 90.00 & 90.00 & 90.00 & 90.00 & $70.706(2)$ \\
\hline$\beta\left[{ }^{\circ}\right]$ & $99.9683(13)$ & $109.318(2)$ & 90.00 & $90.3273(15)$ & $86.720(2)$ \\
\hline $\mathrm{Y}\left[^{\circ}\right]$ & 90.00 & 90.00 & 90.00 & 90.00 & $85.857(2)$ \\
\hline
\end{tabular}


NOT THE PUBLISHED VERSION; this is the author's final, peer-reviewed manuscript. The published version may be accessed by following the link in the citation at the bottom of the page.

\begin{tabular}{|c|c|c|c|c|c|}
\hline compound & 1.0.5 acetone & $2 \cdot \mathrm{CH}_{3} \mathrm{CN}$ & 3 & 4 & 5 \\
\hline$V\left[\AA^{3}\right]$ & $6251.99(14)$ & $3076.30(11)$ & $6487.09(14)$ & $4278.26(13)$ & $1614.65(8)$ \\
\hline$Z$ & 4 & 2 & 8 & 4 & 2 \\
\hline$D_{\text {calcd }}\left[\mathrm{g} \mathrm{cm}^{-3}\right]$ & 1.535 & 1.586 & 1.420 & 1.484 & 1.969 \\
\hline $\begin{array}{l}\lambda[\AA](\mathrm{Cu} \text { or } \\
\text { Mo Ka) }\end{array}$ & 0.7107 & 0.7107 & 1.54178 & 0.7107 & 0.7107 \\
\hline$\mu\left[\mathrm{mm}^{-1}\right]$ & 0.820 & 0.834 & 6.497 & 0.654 & 2.661 \\
\hline abs correction & numerical & numerical & numerical & numerical & numerical \\
\hline$F(000)$ & 2928 & 1488 & 2800.0 & 1952 & 920.0 \\
\hline$\theta$ range $\left[{ }^{\circ}\right]$ & $2.76-29.50$ & $2.27-29.49$ & $3.9-73.6$ & $2.85-29.52$ & $2.78-29.52$ \\
\hline $\begin{array}{l}\text { reflections } \\
\text { collected }\end{array}$ & 72140 & 31390 & 33869 & 48712 & 36367 \\
\hline $\begin{array}{l}\text { independent } \\
\text { reflns }\end{array}$ & $\begin{array}{l}15868 \\
\left(R_{\text {Int }}=0.0326\right)\end{array}$ & $\begin{array}{l}7707 \\
\left(R_{\text {Int }}=0.0343\right)\end{array}$ & $\begin{array}{l}6431 \\
\left(R_{\text {Int }}=0.0473\right.\end{array}$ & $\begin{array}{l}10814 \\
\left(R_{\text {Int }}=0.0324\right)\end{array}$ & $\begin{array}{l}8114 \\
\left(R_{\text {Int }}=0.0371\right)\end{array}$ \\
\hline$T_{\min / \max }$ & $0.727 / 0.827$ & $0.911 / 0.940$ & $0.173 / 0.703$ & $0.904 / 0.947$ & $0.523 / 0.723$ \\
\hline $\begin{array}{l}\text { data/restr/par } \\
\text { am }\end{array}$ & $15868 / 0 / 781$ & $7707 / 0 / 400$ & $6431 / 0 / 372$ & $\begin{array}{l}10814 / 107 / 61 \\
7\end{array}$ & $8114 / 0 / 406$ \\
\hline $\begin{array}{l}\text { goodness-of- } \\
\text { fit on } F^{2}\end{array}$ & 1.030 & 1.033 & 1.070 & 1.085 & 1.073 \\
\hline $\begin{array}{l}R 1^{\mathrm{a}} / w R 2^{\mathrm{b}} \\
{[I>2 \sigma(I)]}\end{array}$ & $0.0301 / 0.0665$ & $0.0330 / 0.0712$ & $\begin{array}{l}0.0331 / 0.088 \\
7\end{array}$ & $0.0408 / 0.0916$ & $0.0317 / 0.0624$ \\
\hline $\begin{array}{l}R 1 / w R 2 \text { (all } \\
\text { data) }\end{array}$ & $0.0384 / 0.0707$ & $0.0451 / 0.0777$ & $\begin{array}{l}0.0378 / 0.092 \\
2\end{array}$ & $0.0493 / 0.0958$ & $0.0448 / 0.0698$ \\
\hline $\begin{array}{l}\text { largest diff } \\
\text { peak/hole/e } \AA \\
-3\end{array}$ & $0.59 /-0.53$ & $0.53 /-0.49$ & $0.70 /-0.76$ & $1.24 /-0.80$ & $1.48 /-1.53$ \\
\hline $21=\Sigma \| F_{0} \mid$ & $\left|F_{\mathrm{c}} \| / \Sigma\right| F_{0} \mid$ & . & & & \\
\hline
\end{tabular}

\section{Results and Discussion}

\section{Synthesis}

Scheme 1 summarizes the preparative routes to the ligands and silver complexes. 
NOT THE PUBLISHED VERSION; this is the author's final, peer-reviewed manuscript. The published version may be accessed by following the link in the citation at the bottom of the page.

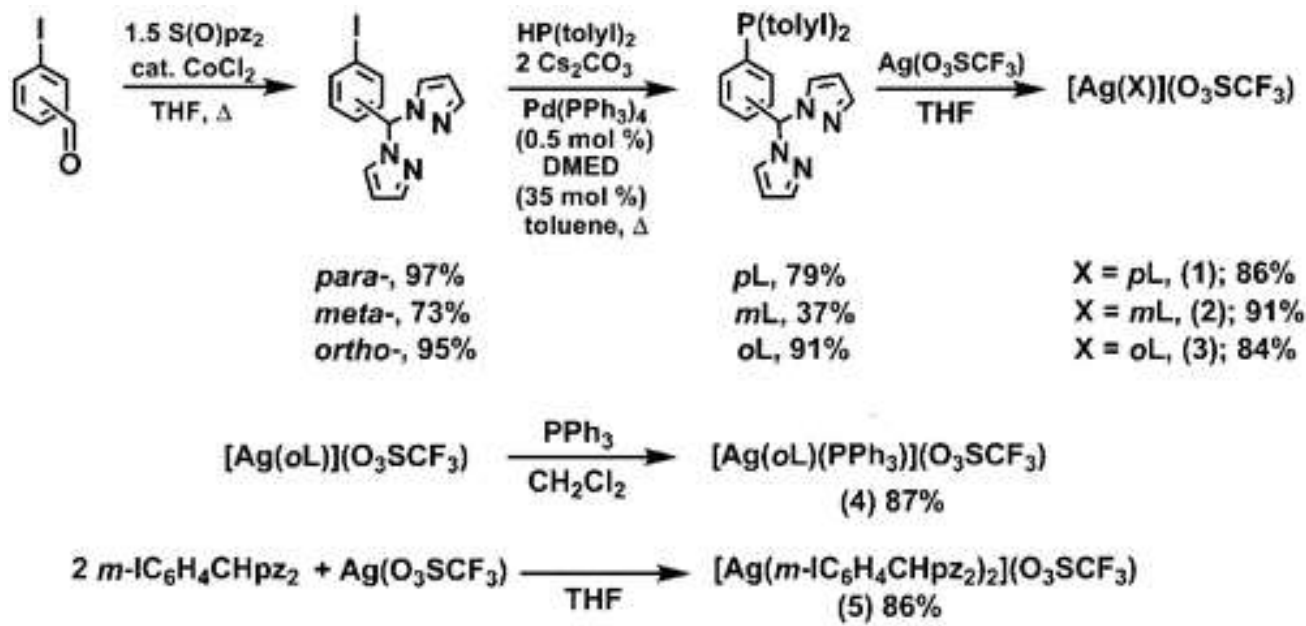

Scheme 1. Preparation of Ligands and Silver Complexes

The di(p-tolyl)phosphine group was introduced to the aryldi(pyrazolyl)methane moiety by a palladium(0)-catalyzed coupling reaction between commercial di $(p$-tolyl)phosphine and the easily prepared isomers of $\left(\mathrm{IC}_{6} \mathrm{H}_{4}\right) \mathrm{CHpz}_{2}$. The di( $p$-tolyl)phosphine group was chosen over the less expensive diphenylphosphine for the convenience of providing relatively simple ${ }^{1} \mathrm{H}$ NMR spectral data (vide infra) compared to a diphenylphosphino analogue. This palladium-catalyzed coupling route proceeded smoothly for the ortho- and paraderivatives. The synthetic yield for the meta- derivative was consistently (significantly) lower than that for the other isomers, an observation for which we do not have a satisfactory explanation. Regardless, the current palladium-catalyzed reaction provides a clean route to the ligands. Alternative, perhaps more conventional, routes to the ligands do not proceed as expected. For instance, attempts at lithium aryl exchange between $n$-butyllithium and haloaryldi(pyrazolyl)methane followed by reaction with diarylphosphine halides or esters gave inseparable mixtures due to competitive deprotonation of the acidic methine and (5-)pyrazolyl hydrogens of the $\left(\mathrm{IC}_{6} \mathrm{H}_{4}\right) \mathrm{CHpz}_{2}$ moiety. Also, the $\mathrm{CoCl}_{2}$-catalyzed Peterson rearrangement ${ }^{21}$ of arylphosphino carboxaldehydes (obtained in low yields) with $\mathrm{S}(\mathrm{O}) \mathrm{pz}_{2}$ did not give any detectable products perhaps due to poisoning of the metal catalyst by binding to the bulky phosphine. The ensuing reactions between THF solutions of silver(I) trifluoromethanesulfonate, $\mathrm{Ag}(\mathrm{OTf})$, and either $\boldsymbol{m} \mathbf{L}$ or $\mathbf{o L}$ in resulted in the immediate precipitation of the desired 1:1 complexes, permission has been granted for this version to appear in e-Publications@Marquette. American Chemical Society does not grant permission for this article to be further copied/distributed or hosted elsewhere without the express permission from American Chemical Society. 
$[\mathrm{Ag}(\boldsymbol{m L})](\mathrm{OTf}), \mathbf{2}$, and $[\mathrm{Ag}(\mathbf{o L})](\mathrm{OTf}), \mathbf{3}$. The analogous reaction with $\boldsymbol{p} \mathbf{L}$ did not form a precipitate in THF, as $[\mathrm{Ag}(\boldsymbol{p} \mathbf{L})](\mathrm{OTf}), \mathbf{1}$, is surprisingly more soluble in THF than the other derivatives. All of the complexes are soluble in $\mathrm{CH}_{3} \mathrm{CN}$ but are insoluble in $\mathrm{Et}_{2} \mathrm{O}$ and hydrocarbon solvents. Complex $\mathbf{1}$ is significantly more soluble than either $\mathbf{2}$ or $\mathbf{3}$ (the latter is nearly insoluble) in $\mathrm{CH}_{2} \mathrm{Cl}_{2}$. In contrast to $\mathbf{1}$ and $\mathbf{2}$, complex $\mathbf{3}$ also shows very low solubility acetone. When 1 equiv of triphenylphosphine is added to a suspension of $\mathbf{3}$ in $\mathrm{CH}_{2} \mathrm{Cl}_{2}$, a solution is obtained immediately upon mixing since $\left[\mathrm{Ag}(\mathrm{oL})\left(\mathrm{PPh}_{3}\right)\right](\mathrm{OTf}), \mathbf{4}$, is formed. The complex $[\mathrm{Ag}(\mathrm{m}-$ $\left.\left.\mathrm{IC}_{6} \mathrm{H}_{4} \mathrm{CHpz}_{2}\right)_{2}\right](\mathrm{OTf}), \mathbf{5}$, was isolated in good yield after collecting the precipitate from mixing THF solutions of the ligand and silver salt.

\section{Solid State}

Complex 1 crystallized as a hemisolvate, 1.0.5 acetone, by vapor diffusion of $\mathrm{Et}_{2} \mathrm{O}$ into an acetone solution. Views of the structure of $\mathbf{1}$ are given in Figure 2. The crystal of $\mathbf{1} \cdot 0.5$ acetone shows a cyclic dimer with two $\mathrm{AgN}_{2} \mathrm{PO}$ kernels that arises by the metal coordinating to one oxygen atom of a triflate anion, two pyrazolyl nitrogens from one bridging ligand, and a phosphorus atom from a second bridging ligand (Figure $2 \mathrm{a}$ ). The average $\mathrm{Ag}-\mathrm{N}_{\mathrm{pz}}$ distance of $2.313 \AA$ is at the lower limit of the 2.3-2.4 $\AA$ range previously found for four-coordinate silver bound to pyrazolyl groups. ${ }^{22}$ The average Ag-P distance of 2.349 $\AA$ is consistent with that found for other silver complexes bound to one phosphine such as $\left(\mathrm{PPh}_{3}\right) \mathrm{AgNO}_{3}(2.369(6) \AA),{ }^{23}\left[\left(\mathrm{PPh}_{3}\right) \mathrm{Ag}\left(\mathrm{O}_{3} \mathrm{SCF}_{3}\right)\right]_{3}$ (avg. 2.345(5), ${ }^{24 \mathrm{a}}$ avg. 2.369(1) $\left.\AA^{24 \mathrm{~b}}\right)$, and $\left[\left(\mathrm{PPh}_{3}\right) \mathrm{AgCl}\right]_{4}$ (2.376(3), $2.388(3) \AA) .{ }^{25}$ The average Ag-O bond length of $2.49 \AA$ is very close to the average value $2.48(13) \AA$ found for other trifluoromethanesulfonate complexes of silver(I $)^{26}$ in a search of the Cambridge Structural Database (CSD), ${ }^{27}$ as detailed in the Supporting Information. Analysis of the bond angles about each silver using Hauser's four-coordinate geometry index, ${ }^{28} \mathrm{~T}_{4}=\left[360^{\circ}-(a+\beta)\right] / 141^{\circ}$ $=0.67$ for $\mathrm{Ag} 1$ and 0.70 for Ag2 where $a$ and $\beta$ are the largest angles about each silver (138 and $128^{\circ}$ for Ag1; 135 and $126^{\circ}$ for Ag2), indicates that the coordination geometry is seesaw shaped; a $\mathrm{T}_{4}$ value of zero would correspond to a tetrahedron, while a value of 1 would indicate square planar geometry. It is also of interest to note that the dimer formed from two bridging ligands and two silvers is 
characterized by an 18-member $\left(\mathrm{Ag}_{2} \mathrm{C}_{10} \mathrm{~N}_{4} \mathrm{P}_{2}\right)$ metallacycle ring that has a silver-silver separation of $7.65 \AA$ and has approximate local $C_{2}$ symmetry of framework (ring) atoms (Figure $2 b, c$ ). The conformation of the metallacycle is such that there are two types of $p$-tolyl groups that can be classified as either "axial" (thicker green rings bottom part of Figure 2c) or "equatorial" (thicker violet rings in Figure 2b). The "axial" $p$-tolyl groups are on the same side of the metallacycle ring. Similarly there are two types of pyrazolyl groups, occupying either "axial" or "equatorial" positions with respect to the metallacycle. The "axial" pyrazolyls are found on the same side of the metallacyle ring but on the side opposite of the "axial" $p$-tolyl groups. The 18-member metallacycle deviates from perfect $C_{2}$ symmetry as detected by measurement of the $\mathrm{AgP}-\mathrm{C}_{\text {meth }} \mathrm{Ag}$ torsion angle, $\mathrm{T}_{1}$ (pink lines, Figure $2 d$ ), associated with each ligand ( $T_{1}=71$ and $77^{\circ}$, for ligands $A$ and $B$, respectively); an acute angle indicates the two silver atoms are on the same side of the central phenylene linker. The $\mathrm{T}_{1}$ torsion angle can also be decomposed into two components based on the relative disposition of the silver-bound di(p-tolyl)phosphine or di(pyrazolyl)methane unit with respect to the phenyl group that links the moieties. That is, one torsion angle, $\mathrm{T}_{\mathrm{a}}$ (green lines, Figure $2 \mathrm{~d}$ ) defined as the acute angle associated with four atoms $\mathrm{AgP}-\mathrm{C}_{\mathrm{ipso}} \mathrm{C}_{\text {ortho, }}$ essentially describes the rotation of the Ag-P bond from the mean plane of the phenyl linker. A second torsion angle, $\mathrm{T}_{\mathrm{b}}$ (blue lines, Figure $2 \mathrm{~d}$ ) defined as the acute angle of four atoms, $\mathrm{AgC} \mathrm{C}_{\text {meth }}-\mathrm{C}_{\mathrm{ipso}} \mathrm{C}_{\text {ortho, }}$ provides a measure of the rotation of the $\mathrm{Ag}-\mathrm{C}_{\text {meth }}$ vector from coincidence with the mean plane of the phenyl linker (approximated by the $\mathrm{C}_{\mathrm{ipso}}-\mathrm{C}_{\text {ortho }}$ bond). Negative values for $\mathrm{T}_{\mathrm{a}}$ and $\mathrm{T}_{\mathrm{b}}$ indicate a clockwise rotation of the Ag-P or Ag$\mathrm{C}_{\text {meth }}$ vector from the plane of the phenylene linker (again, approximated by the $\mathrm{C}_{\mathrm{ipso}}-\mathrm{C}_{\text {ortho }}$ bond); positive values describe a counter-clockwise rotation. The $T_{1}, T_{a}$, and $T_{b}$ values for one ligand (" $A$ ", containing P1) are $71.1,-12.0$, and $80.6^{\circ}$, respectively, whereas those values for the other ligand (" $\mathrm{B}$ ", containing P2) are 77.2, -41.7 , and $-67.4^{\circ}$. The different $T_{a}$ (or $T_{b}$ ) values found for ligands $A$ and $B$ of the metallacycle are sufficient to exclude any symmetry relation between ligands. 
NOT THE PUBLISHED VERSION; this is the author's final, peer-reviewed manuscript. The published version may be accessed by following the link in the citation at the bottom of the page.

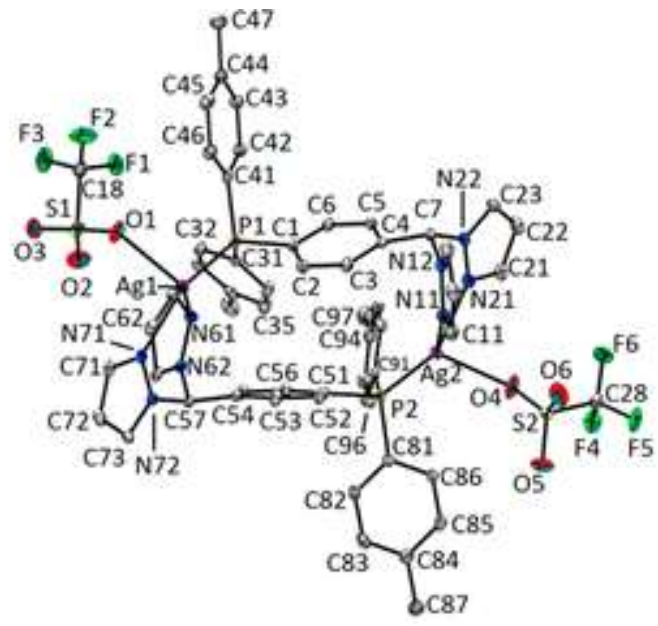

(a)

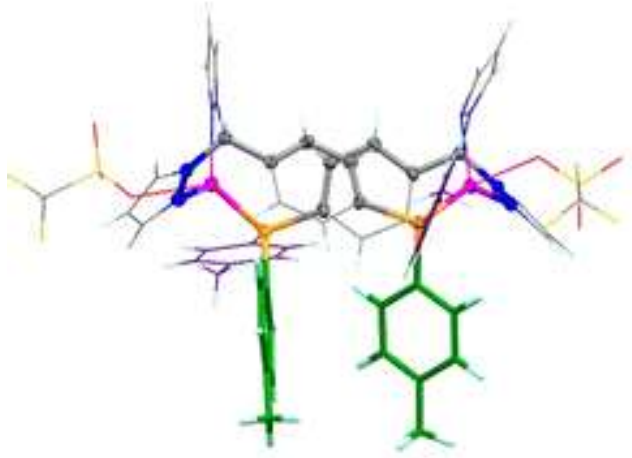

(c)

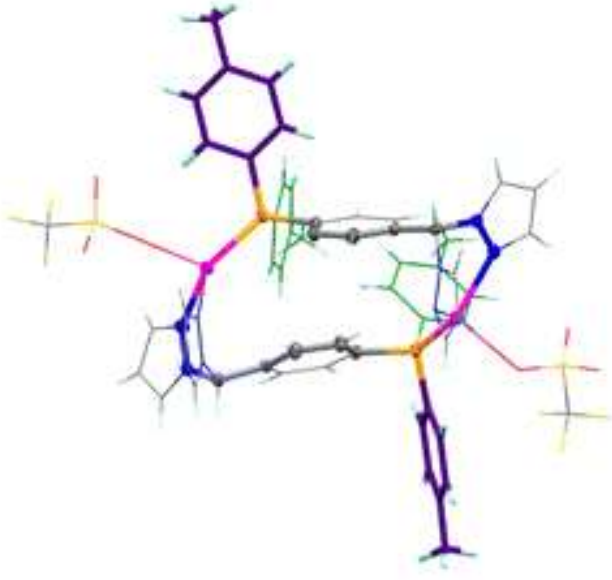

(b)

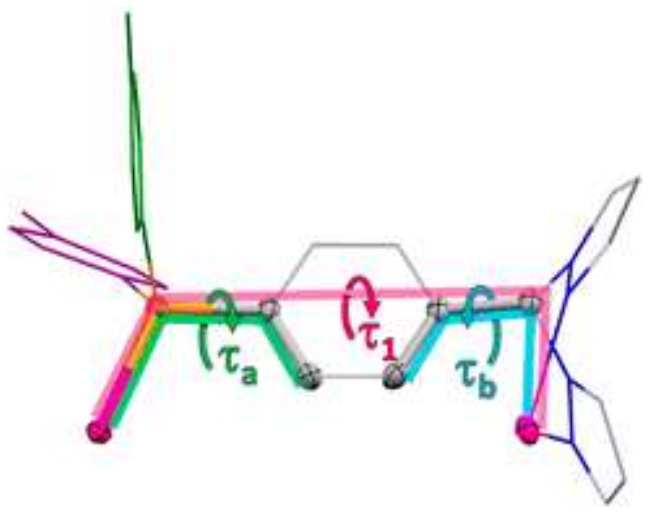

(d)

Figure 2. Views of the structure of $\mathbf{1} \cdot 0.5$ acetone with solvent removed for clarity. (a) Approximately down the $b$-axis; (b) 18-member metallacycle, approximately down the $C_{2}$ axis, with equatorial tolyl groups highlighted (violet capped sticks); (c) orthogonal to approximate $C_{2}$ axis with axial tolyl groups highlighted (green capped sticks). (d) Labeling scheme of various torsion angles. Selected bond distances $(\AA)$ : Ag1-P1 2.3555(5), Ag1-O1 2.5125(14), Ag1-N61 2.3336(15), Ag1-N71 2.3094(15), Ag2-P2 2.3445(5), Ag2-O4 2.4734(16), Ag2-N11 2.2713(15), Ag2-N21 2.3361(16). Selected bond angles $\left({ }^{\circ}\right)$ : P1-Ag1-O1 108.27(4), N61-Ag1-P1 127.90(4), N61-Ag1-O1 92.17(5), N71-Ag1-P1 137.84(4), N71-Ag1-O1 97.21(5), N71-Ag1-N61 82.54(5), P2-Ag2-O4 110.51(5), N11-Ag2-P2 135.10(4), N11-Ag2-O4 105.59(6), N11-Ag2N21 82.67(5), N21-Ag2-P2 126.11(4), N21-Ag2-O4 84.20(6).

Complex 2 crystallizes as a $\mathrm{CH}_{3} \mathrm{CN}$ solvate, $\mathbf{2} \cdot \mathrm{CH}_{3} \mathrm{CN}$, after vapor diffusion of $\mathrm{Et}_{2} \mathrm{O}$ into an acetonitrile solution. In this case, a cyclic dimeric dication is formed from two ligands bridging two silver centers with an Ag $\cdots A g$ separation of $6.003 \AA$ (Figure 3a); the triflate anions are not bound to silver centers ${ }^{29}$ as was the previous case. The dication has inversion ( $C_{i}$ point group) symmetry, and thus each silver

Inorganic Chemistry, Vol 53, No. 22 (2014): pg. 12108-12121. DOI. This article is @ American Chemical Society and permission has been granted for this version to appear in e-Publications@Marquette. American Chemical Society does not grant permission for this article to be further copied/distributed or hosted elsewhere without the express permission from American Chemical Society. 
has an identical planar " $\mathrm{Y}$-shaped" $\mathrm{AgN}_{2} \mathrm{P}$ coordination environment (sum of angles about $\mathrm{Ag}=360^{\circ}$ ). The average $\mathrm{Ag}-\mathrm{N}_{\mathrm{pz}}$ bond distance of $2.28 \AA$ is at the upper limit of the $2.2-2.3 \AA$ range found for tricoordinate silver (bound to pyrazolyl donors), ${ }^{22}$ and the Ag-P bond distance of $2.3445(6)$ is nearly identical to the average distance found for $\mathbf{1} \cdot 0.5$ acetone, which demonstrates previous observations that the Ag-P distance depends mainly on the number of phosphines bound to silver and to a lesser extent on the coordination number of silver. ${ }^{30}$ The dication also possesses a 16-member $\mathrm{Ag}_{2} \mathrm{C}_{8} \mathrm{~N}_{4} \mathrm{P}_{2}$ metallacylic ring. There are two types of pyrazolyl groups, "axial" and "equatorial", where "axial" pyrazolyls project further above and below the mean plane of the 16-member metallacycle ring than the "equatorial" pyrazolyls, right of Figure 3b. The ring can be said to be in a chair conformation where either "equatorial" or "axial" pyrazolyl nitrogens serve as the "head" and "foot" of the chair. There are also two types of p-tolyl groups, "axial" (green, Figure $3 \mathrm{~b}$ ) and "equatorial" (violet, Figure 3b), but the "axial" $p$-tolyl groups are on opposite faces of the mean plane of the metallacycle. Finally, as a means of comparison with $\mathbf{1}$, the $T_{1}, T_{a}$, and $T_{b}$ values for each ligand of the dication in $\mathbf{2}$ are $-25.2,+51.7,+79.4$ for one ligand and $+25.2,-51.7,-79.4$ for the other. The acute $T_{1}$ values in $\mathbf{2}$ indicate that the two silvers are on the same side of the central phenylene linker, while the identical magnitudes but opposite signs of the $T_{1}, T_{a}$, and $T_{b}$ values indicate symmetry equivalence (by inversion) of the two ligands. not grant permission for this article to be further copied/distributed or hosted elsewhere without the express permission from American Chemical Society. 


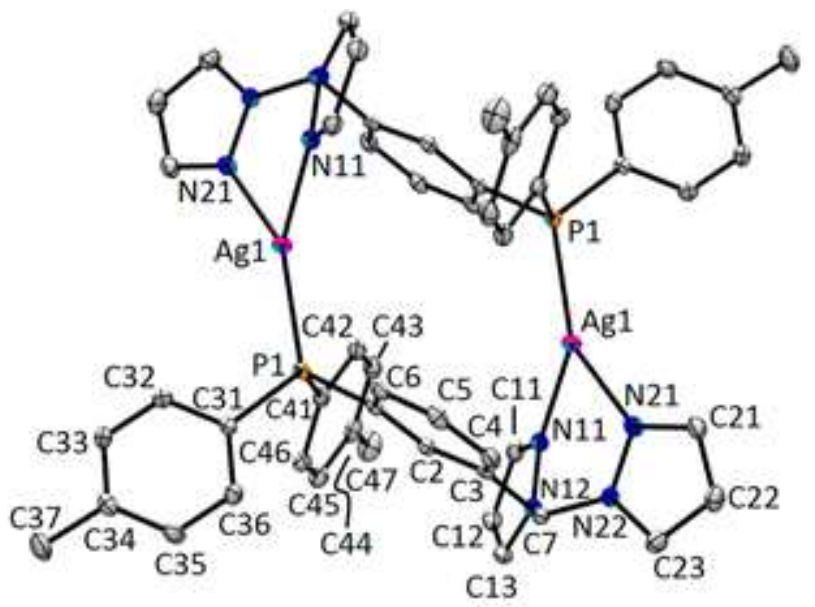

(a)

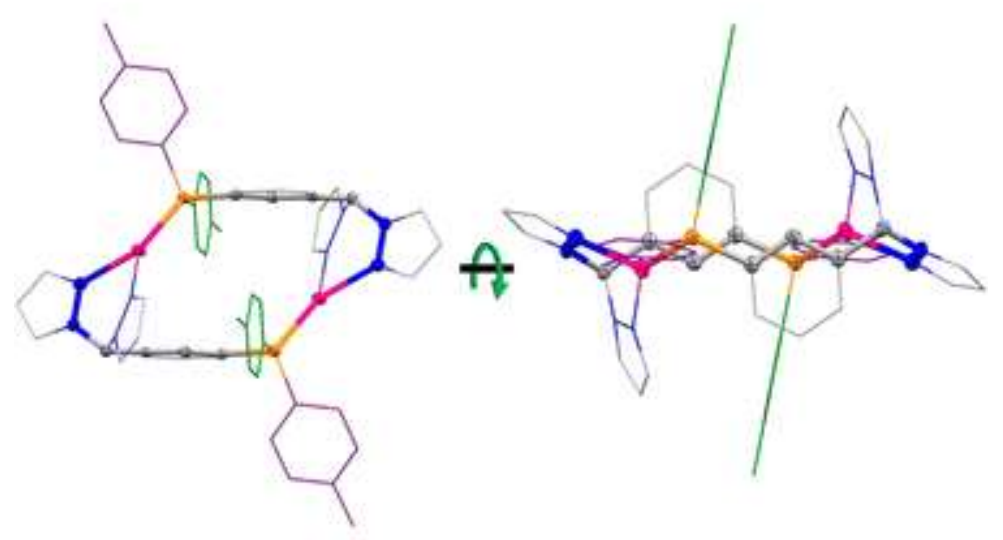

(b)

Figure 3. Structure of $\mathbf{2} \cdot \mathrm{CH}_{3} \mathrm{CN}$. (a) A dimeric dication with atom labeling. Hydrogen atoms, triflate anion and acetonitrile solvate molecule removed for clarity. (b) Views emphasizing 16-member metallacyclic ring and symmetrically distinct $p$-tolyl groups (green and violet). Selected bond distances $(\AA)$ : Ag1-P1 2.3445(6), Ag1-N11 2.2564(18), Ag1-N21 2.3043(19). Selected bond angles ( $\left.{ }^{\circ}\right)$ : N11-Ag1-P1-144.04(5), N11-Ag1-N21 82.90(7), N21-Ag1-P1 132.63(5).

Complex 3 crystallizes in the orthorhombic space group $P b c a$ after vapor diffusion of $\mathrm{Et}_{2} \mathrm{O}$ into an acetonitrile solution. The asymmetric unit consists of one ligand, one silver, and one triflate anion, Figure $4 \mathrm{a}$. The ligand again acts in a bridging manner by binding one silver center with the phosphorus atom and another silver with two nitrogens of the $\mathrm{CHpz}_{2}$ moiety. In contrast to the previous cases, the obtuse $\mathrm{T}_{1}$ torsion angle of $151.5^{\circ}$ in $\mathbf{3}$ indicates that the silver centers are on opposite faces of the phenylene group that separates the phosphino and di(pyrazolyl)methane groups. This disposition of donor groups results in a coordination polymer instead of

Inorganic Chemistry, Vol 53, No. 22 (2014): pg. 12108-12121. DOI. This article is @ American Chemical Society and permission has been granted for this version to appear in e-Publications@Marquette. American Chemical Society does not grant permission for this article to be further copied/distributed or hosted elsewhere without the express permission from American Chemical Society. 
a cyclic species, Figure 4b. The coordination polymer propagates along the $b$-direction. Specifically, the polymer is generated by translation of the asymmetric unit along the $b$-glide plane perpendicular to the $a$ axis (i.e., with reflection through the ac-plane). The silver centers can be considered either three- or four-coordinate depending on one's view of the long Ag1-01 contact of 2.809(3) $\AA$, Figure 4c. We favor tetracoordination because this distance is $0.43 \AA$ less than the sum of the van der Waals radii $(3.24 \AA$ ) and is within $3 \sigma$ of the average $A g-O$ distance of $2.48(13) \AA$ found from the CSD (Figure S2, Supporting Information), and since the average $A g-\mathrm{N}_{\mathrm{pz}}$ distance of $2.33 \AA$ is in the 2.3-2.4 $\AA$ range found for other tetracoordinate silver complexes with pyrazolyl-ligands. ${ }^{22}$ If the silver is considered tetracoordinate, then the four-coordinate geometry index, $t_{4}=0.60$, indicates a seesaw geometry. There are two other contacts, Ag1-02 3.055(2) $\AA$ and Ag1H7 2.82, that are about $0.2 \AA$ less than the sum of the respective van der Waals radii and, as such, can be considered secondary interactions. 


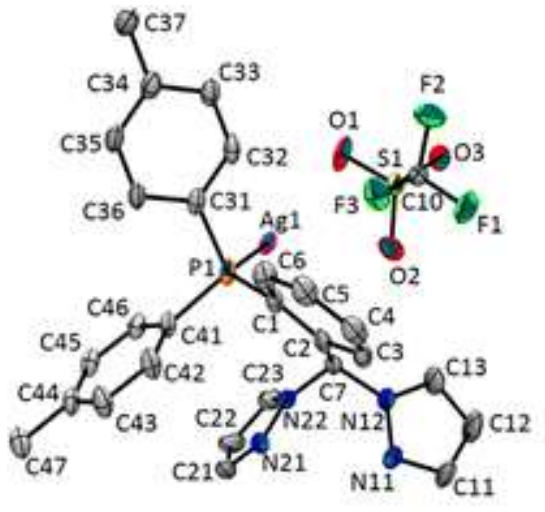

(a)

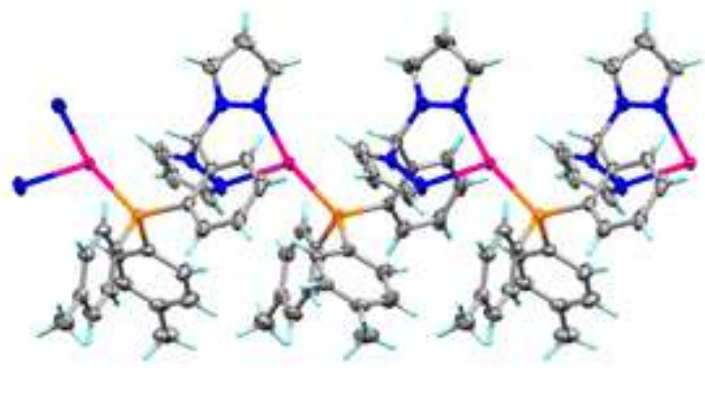

(b)

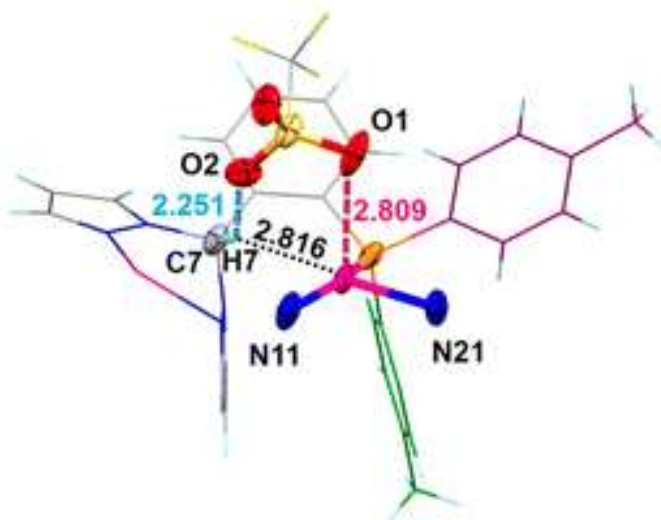

(c)

Figure 4. Structure of $[\mathrm{Ag}(\mathrm{OL})](\mathrm{OTf})$, 3. (a) Asymmetric unit with atom labeling and with hydrogen atoms removed for clarity. (b) View down the a-axis of a chain propagating along the $b$-direction, triflate anions removed for clarity. (c) View of primary and secondary coordination sphere around silver with interatomic distances in $\AA$. Selected bond distances $(\AA)$ : Ag1-P1 2.3839(6), Ag1-N11 2.266(2), Ag1-N21 2.394(2), Ag1-01 2.809(3). Selected bond angles $\left({ }^{\circ}\right)$ : P1-Ag1-N21 113.53(6), N11Ag1-P1 160.73(7), N11-Ag1-N21 83.37(8), P1-Ag1-O1 99.67(5), N11a-Ag1-O1 89.85(9), N21a-Ag1-01 87.57(8).

The structure of 4 (Figure 5) consists of a monomeric species that has tetracoordinate silver bound to a triphenylphosphine, to $\mathbf{o L}$ in a chelating $K^{2} N, P$ - manner, and to an oxygen of the triflate anion (in a $\mathrm{K}^{1}$ - fashion). The structure is afflicted by disorder that allows limited discussion of the coordination environment but precludes extensive analysis of the supramolecular structure. That is, the pyrazolyl ring that is not bound to the silver is rotationally disordered about the C7N22(a) bond equivalently over two positions. The triflate anion is 
located in two nearby positions in an 88:12 ratio, favoring the position depicted on the left of Figure 5 with two oxygen atoms of the anion oriented toward the triphenylphosphine group. The $\mathrm{Ag}-\mathrm{O}$ distance of either disorder component (Ag1-01 2.461(3), Ag1-01a 2.323(17) $\AA$ ) is shorter than the $2.48 \AA$ average $\mathrm{Ag}-\mathrm{O}$ (triflate) distance from the above CSD database search, which permits a confident assertion of $\mathrm{K}^{1} \mathrm{O}$ - coordination. The $\mathrm{Ag}-\mathrm{N}_{\mathrm{pz}}$ distance of $2.408(2) \AA$ is in line with tetracoordinate silver. The Ag1-P1 distance of 2.5060(6) $\AA$ is longer than those distances in 1-3 but is consistent with four-coordinate silver complexes of $\mathrm{P}(p \text {-tolyl })_{3} .{ }^{31}$ The $\mathrm{Ag}-\mathrm{P} 2\left(\mathrm{PPh}_{3}\right)$ distance of 2.4186(6) $\AA$ is in line with the ca. $2.42 \AA$ average Ag-P distance found in other tetracoordinate silver complexes with two triarylphosphine ligands. ${ }^{30,32}$
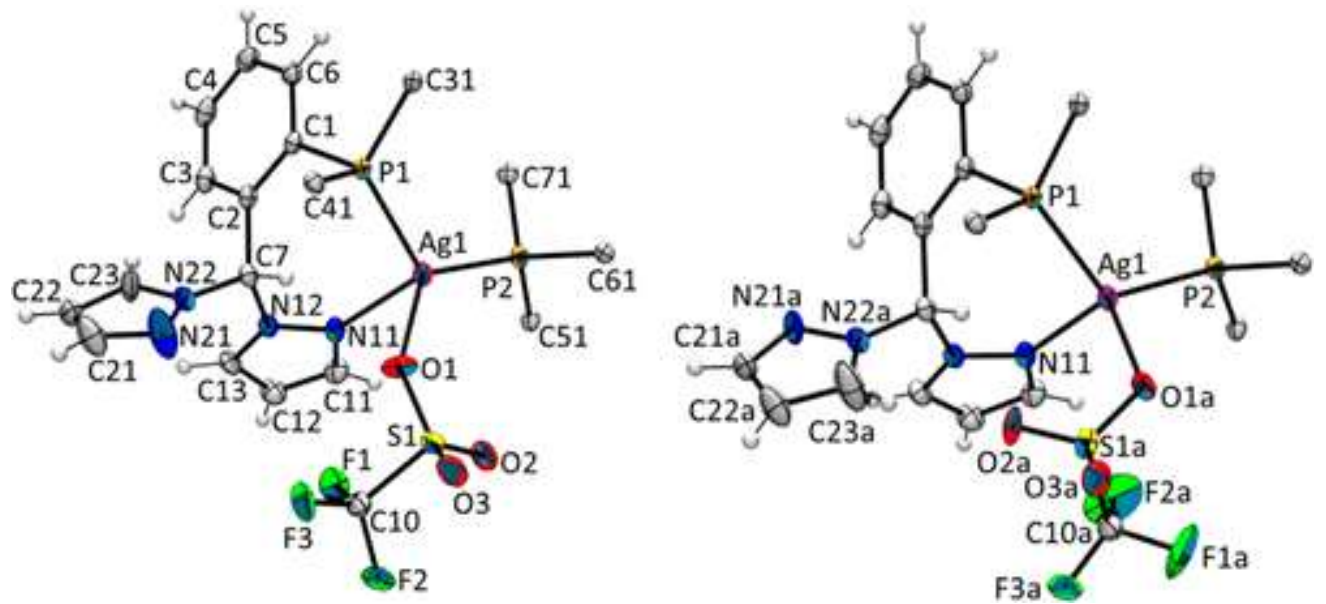

Figure 5. Views of two disorder components in the structure of $\left[\mathrm{Ag}(\mathbf{O L})\left(\mathrm{PPh}_{3}\right)\right](\mathrm{OTf})$, 4, with atom labeling. Only the ipso-carbon atom bound to the phosphine atom on of the tolyl groups of $\boldsymbol{o} \mathbf{L}$ and on the phenyl rings in $\mathrm{PPh}_{3}$ are shown for clarity. Selected bond distances $(\AA)$ : Ag1-P1 2.5060(6), Ag1-P2 2.4186(6), Ag1-N11 2.408(2), Ag1O1 2.461(3), Ag1-O1a 2.323(17). Selected bond angles ( $\left.{ }^{\circ}\right)$ : P2-Ag1-P1 124.13(2), P2-Ag1-O1 131.42(7), O1-Ag1-P1 92.39(6), O1a-Ag1-P1 102.0(5), O1a-Ag1-P2 106.0(4), O1a-Ag1-N11 111.8(4), N11-Ag1-P1 105.25(5), N11-Ag1-P2 107.59(5), N11-Ag1-O1 89.19(8).

\section{Supramolecular Structures}

Aside from fundamental interest, the analysis of the supramolecular structures of complexes $\mathbf{1}$-acetone, $\mathbf{2} \cdot \mathrm{CH}_{3} \mathrm{CN}, \mathbf{3}$, and $\mathbf{5}$ is of importance to the interpretation of their solid state IR spectrum and potentially to the powder X-ray diffraction (PXRD) studies described later. The supramolecular structure of $\mathbf{1}$-acetone is shown in 
Figure 6 and is discussed below, while analyses of the other structures are provided in the Supporting Information. A feature common to the supramolecular structures of all the current complexes, and to most other $\mathrm{Ag}(\mathrm{OTf})$ complexes of di(pyrazolyl)methane ligands, ${ }^{8 \mathrm{a}}$ is $\mathrm{CH} \cdots \mathrm{O}$ weak hydrogen bonding interactions ${ }^{33}$ that occur between the triflate anion and the acidic methine and 5-pyrazolyl hydrogen atoms. That is, in 1-acetone, a variety of weak $\mathrm{CH} \cdots$ O hydrogen bonding interactions organize the 3D structure into stacked bilayer sheets with channels along the $b$-axis that hold solvent. Figure 6 shows only the shortest (sum of van der Waals radii $-0.2 \AA$ ) and presumably strongest of these "intermolecular" $\mathrm{CH}$...O interactions (cyan dashed lines), while Table 2 collects the metrics of these and of the longer interactions. Of the two independent triflate anions, that with $\mathrm{S} 2$ serves as a bridge to connect neighboring dications along the a-direction to form a dimer of dications (top right of Figure 6). That is, one of the oxygens (04) interacts with $\mathrm{Ag} 2$ of one cation, while $\mathrm{O} 5$ acts as an acceptor in a bifurcated weak hydrogen bonding interaction with the methine (H57) and 5-pyrazolyl (H67) hydrogen donors of a neighboring dication with the metrics listed in Table 2. The third oxygen atom (O6) of this triflate anion participates in a long and presumably very weak noncovalent bonding interaction with a tolyl ring hydrogen ( $\mathrm{H} 85$, ortho- to the methyl group). The Ag1-01 bonds anchor two of the other independent triflate anions (that each contain S1) to the dimer of dications. The oxygen atom $\mathrm{O} 3$ of each "S1-containing" triflate acts as an acceptor in a bifurcated $\mathrm{CH} \cdots \mathrm{O}$ interaction with the methine hydrogen ( $\mathrm{H} 7$ ) of neighboring dimer of dications as well as with a methyl group hydrogen (H3Sa) of an acetone solvate molecule. A bilayer sheet structure, that is one unit cell in width along the $a-$ direction but infinite in the bc-plane (Figure 6), is formed since each dimer of dications contains two hydrogen donors and two acceptors of four $\mathrm{C} 7 \mathrm{H} 7 \cdots \mathrm{O} 3$ interactions. The $b c$-sheets are further connected along the a-direction by two types of $\mathrm{CH} \cdots \mathrm{O}$ interactions involving the acetone solvate molecule. The first interaction, C3SH3Sa…03, described previously, holds the acetone molecule to one sheet, while the oxygen atom of acetone (O1S) acts as an acceptor to a phenylene hydrogen ( $\mathrm{H6}$ ) that is ortho- to the ditolylphosphine group (top left of Figure 6) of an adjacent bilayer sheet. As a result of the sheet stacking, there are channels along the $b$-direction that contain acetone molecules as shown in the two views in the bottom of Figure 6. 
NOT THE PUBLISHED VERSION; this is the author's final, peer-reviewed manuscript. The published version may be accessed by following the link in the citation at the bottom of the page.

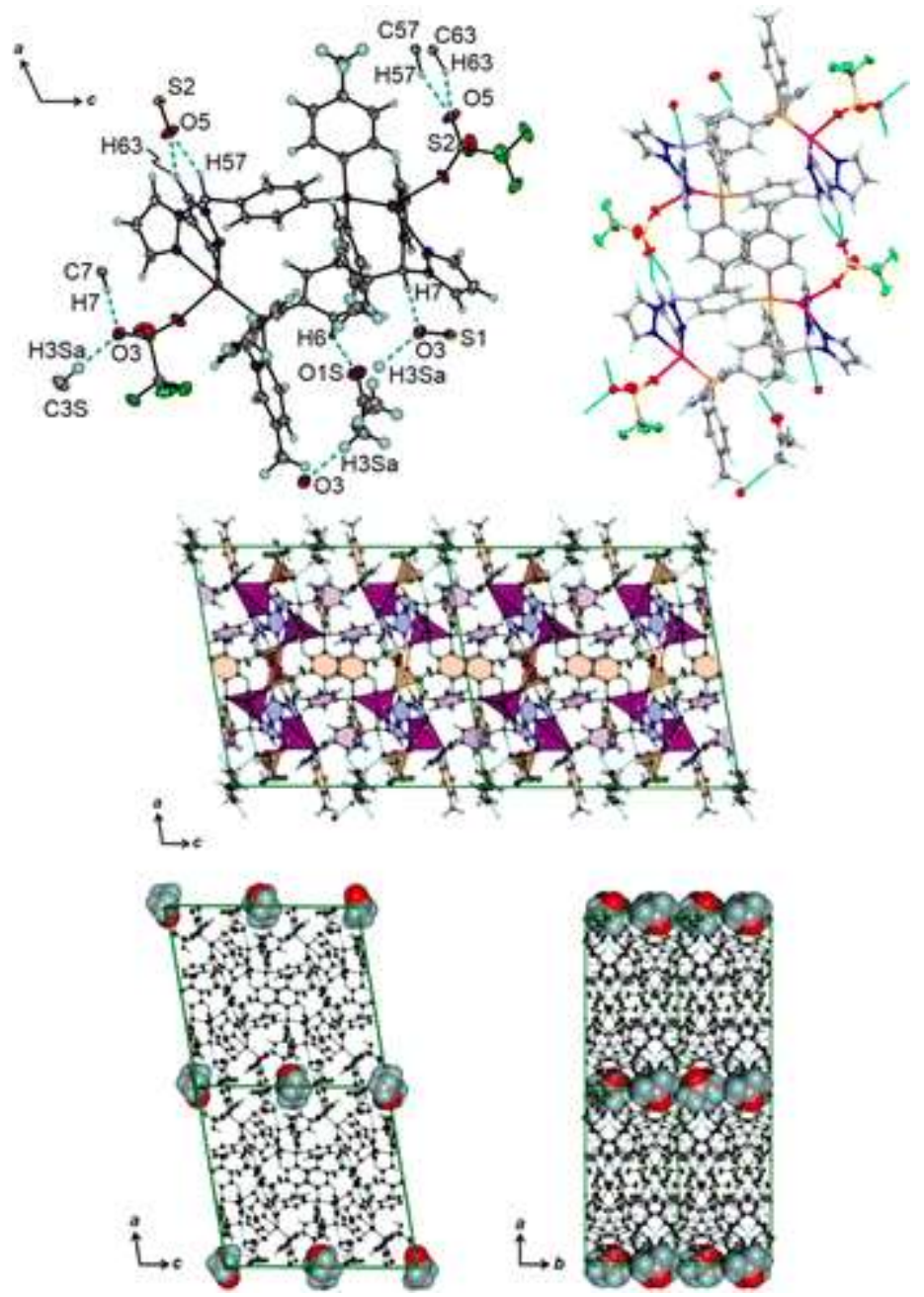

Figure 6. Supramolecular structure of $\mathbf{1} \cdot 0.5$ acetone. Top left: View of $\mathrm{CH} \cdots \mathrm{O}$ interactions (cyan dashed lines); top right: Dimer formed by interactions involving only 05. Middle: View of $b c$-sheet bilayer structure ( $\mathrm{Ag}$ as pink tetrahedra, triflate $\mathrm{SO}_{3} \mathrm{C}$ unit as yellow tetrahedra, pyrazolyl rings as blue pentagons, phenylene linkers as violet hexagons; tolyl rings as orange hexagons); bottom: View of four unit cells down $b$ - (left) and down $c$ - (right) showing acetone filling channels along the $b$ direction.

Table 2. Geometries of $\mathrm{C}-\mathrm{H} \cdots \mathrm{O}$ Weak Hydrogen-Bonding Interactions in $\mathbf{1} \cdot$ acetone

\begin{tabular}{|c|c|c|c|c|}
\hline Donor(D)(-H) ․Acceptor(A) & D-H $(\AA)$ & 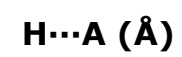 & $\mathbf{D} \cdots A(\AA)$ & $D-H \cdots A\left({ }^{\circ}\right)$ \\
\hline \multicolumn{5}{|c|}{$\mathrm{C}-\mathrm{H} \cdots \mathrm{O}$ interactions } \\
\hline $\mathrm{C} 7-\mathrm{H} 7 \cdots \mathrm{O} 3$ & 1.00 & 2.14 & $3.130(2)$ & 169 \\
\hline $\mathrm{C} 23-\mathrm{H} 23 \cdots \mathrm{O} 1$ & 0.95 & 2.53 & $3.368(2)$ & 147 \\
\hline $\mathrm{C} 57-\mathrm{H} 57 \cdots \mathrm{O} 5$ & 1.00 & 2.21 & $3.119(2)$ & 150 \\
\hline $\mathrm{C} 63-\mathrm{H} 63 \cdots \mathrm{O} 5$ & 0.95 & 2.40 & $3.133(2)$ & 134 \\
\hline $\mathrm{C} 85-\mathrm{H} 85 \cdots \mathrm{O}$ & 0.95 & 2.59 & $3.484(2)$ & 158 \\
\hline C3S-H3Sa‥O3 & 0.98 & 2.47 & $3.432(3)$ & 169 \\
\hline
\end{tabular}

Inorganic Chemistry, Vol 53, No. 22 (2014): pg. 12108-12121. DOI. This article is @ American Chemical Society and permission has been granted for this version to appear in e-Publications@Marquette. American Chemical Society does not grant permission for this article to be further copied/distributed or hosted elsewhere without the express permission from American Chemical Society. 
NOT THE PUBLISHED VERSION; this is the author's final, peer-reviewed manuscript. The published version may be accessed by following the link in the citation at the bottom of the page.

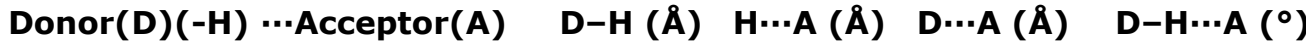 $\mathrm{C} 6-\mathrm{H} 6 \cdots \mathrm{O} 1 \mathrm{~S}$ \\ $0.95 \quad 2.36$ \\ $3.224(3) \quad 151$}

It is important to note that each oxygen of both types of triflate ion in $\mathbf{1}$-acetone (or of the anions in $\mathbf{2} \cdot \mathrm{CH}_{3} \mathrm{CN}, \mathbf{3}, \mathbf{4}$, or $\mathbf{5}$, see Supporting Information) experiences a distinct (weak hydrogenbonding) environment in the solid state. If the $\mathrm{C}-\mathrm{H} \cdots \mathrm{O}$ interactions are significant, the local symmetry around either S1 or S2 in 1-acetone could be effectively lowered to $C_{1}$ and give rise to an increase in the number and/or broadness of S-O stretches in the solid state IR spectrum (vide infra) compared to the idealized case where the local symmetry about sulfur in the $\mathrm{CF}_{3} \mathrm{SO}_{3}$ group is $C_{3 v}$.

\section{PXRD}

Powder X-ray diffraction data were collected for the solid samples of 1-3 and $\mathbf{5}$ obtained immediately after their initial isolation (as-prepared samples are precipitated from THF and/or washed with $\mathrm{Et}_{2} \mathrm{O}$ and dried under a vacuum), and after recrystallization from various solvents followed by drying under a vacuum. The PXRD patterns obtained for as-prepared samples of $\mathbf{5}$ match those calculated from single-crystal X-ray diffraction data (Figure S6, Supporting Information) which is expected since this compound crystallized without solvent in the lattice. This contrasts the situation for $\mathbf{2}$ where the diffraction pattern of the solvent-free (combustion analysis) powder does not match that calculated for the single crystal of 2. $\mathrm{CH}_{3} \mathrm{CN}$ (Figure $\mathrm{S6}$ ), as might be expected. While X-ray diffraction quality crystalline blocks of $\mathbf{1} \cdot 0.5$ acetone were obtained after allowing a layer of hexanes to diffuse into an acetone solution of $\mathbf{1}$, vapor diffusion of $\mathrm{Et}_{2} \mathrm{O}$ into an acetonitrile solution of $\mathbf{1}$ produces microcrystalline, ultrathin needles that are unsuitable for single crystal $X$-ray diffraction. Simply air drying these ultrathin needles is sufficient to give a solvent-free sample of $\mathbf{1}$ as determined by combustion analysis. Samples of air-dried needles of $\mathbf{1}$ (from $\mathrm{CH}_{3} \mathrm{CN}$ ) and those as-prepared samples of $\mathbf{1}$ from THF showed variable levels of crystallinity (middle and top of Figure 7, respectively). Given the supramolecular structure of $\mathbf{1} \cdot 0.5$ acetone that showed solvent partly occupying channels, the ease of solvent removal, and the similarity of PXRD patterns, it is likely that the structures of the crystalline samples 
obtained from other solvent systems (i.e., especially the microcrystalline needles from $\mathrm{CH}_{3} \mathrm{CN}$ ) are closely related to that of $\mathbf{1} \cdot 0.5$ acetone. Similarly, the crystal of $\mathbf{3}$ had heavily disordered solvent ( $\mathrm{Et}_{2} \mathrm{O}: \mathrm{CH}_{3} \mathrm{CN}$ ) in large voids in the crystal (Supporting Information). Yet, the calculated PXRD pattern matched that of the asisolated, solvent-free (combustion analysis) powder indicating that the overall structural features remain intact regardless of the presence or absence of solvent.

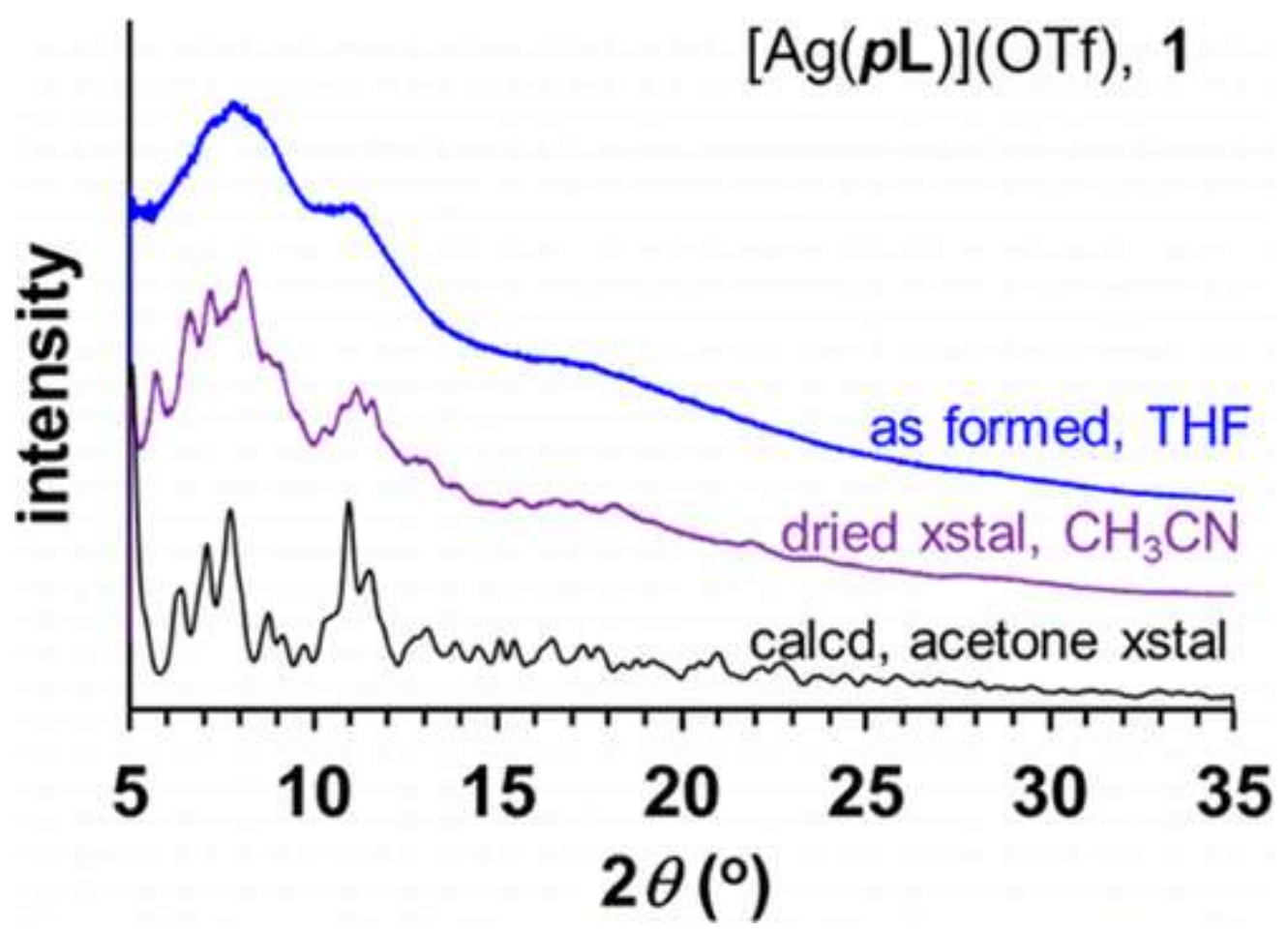

Figure 7. PXRD patterns obtained for samples of 1 obtained under various conditions compared to data (bottom) calculated from the single crystal diffraction data for $\mathbf{1} \cdot 0.5$ acetone.

\section{IR Spectra}

The IR spectra of $\mathbf{1 - 4}$ and $\mathbf{5}$ in $\mathrm{CH}_{3} \mathrm{CN}$ show characteristic bands for "unbound" or "ionic" triflate anions at 1271, 1157, 1093, and 966 $\mathrm{cm}^{-1}$, for $\mathrm{SO}_{3}$ and $\mathrm{CF}_{3}$ stretching modes and combination bands, ${ }^{34}$ but the solid state spectra are much more complicated than expected (Figure S7) presumably due to the various different weak $\mathrm{CH} \cdots \mathrm{O}$ interactions and, in the case of $\mathbf{1}$, to the two crystallographically distinct types of triflate anions in the unit cell. For instance, single 
crystal X-ray diffraction of $\mathbf{5}$ shows tetracoordinate silver $\left(\mathrm{T}_{4}=0.73\right.$, seesaw $\mathrm{AgN}_{4}$ geometry, see Figure S1) due to binding only to pyrazolyl nitrogen donors. In this case, the triflate anion is not bound to the metal center, yet the solid-state IR spectrum is complicated.

Examination of the supramolecular structure reveals that each of the three oxygens of the triflate participates in a different type of $\mathrm{CH} \cdots \mathrm{O}$ interaction (Figure S5) which may effectively lower the local symmetry of the $\mathrm{SO}_{3}$ moiety and give rise to a greater than expected number of S-O stretches. The IR spectra of as-isolated powders of 1-4 and $\mathbf{5}$ are identical to those obtained for air-dried crystals; combustion analyses indicate that the powders or air-dried crystals are solvent-free.

\section{Solution}

NMR

The variable temperature multinuclear NMR and ESI(+) MS data (vide infra) indicate that, in solution at room temperature, compounds $\mathbf{1}$ and $\mathbf{2}$ likely maintain cyclic structures similar to the solid state, whereas the solution structures of $\mathbf{3}$ and $\mathbf{4}$ are different than their solid-state structures. The ${ }^{31} \mathrm{P}$ NMR spectral data of triarylphosphine silver(I) complexes are particularly useful for characterization as they can be diagnostic of the number and type of phosphine ligands bound to silver. ${ }^{30,35}$ Silver has two naturally occurring NMR-active $(I=1 / 2)$ nuclei, ${ }^{107} \mathrm{Ag}$ (52\% abundance) and ${ }^{109} \mathrm{Ag}$ (48\% abundance). When silver is bound to one phosphorus atom, a characteristic set of doublet resonances such as shown in Figure $8 a$ is expected. In the absence of exchange, the nearly overlapping doublets are resolved, and the ratio of one-bond coupling constants ${ }^{1} J_{109 \mathrm{Ag}-\mathrm{P}} /{ }^{1} J_{107 \mathrm{Ag}-\mathrm{P}}$ should be 1.15 , corresponding to the ratio of the nuclear magnetic moments $\left(\mu_{\mathrm{I}}\right.$, in nuclear magneton units, $\left.\mu_{\mathrm{N}}\right) \mu_{\mathrm{I}}\left({ }^{109} \mathrm{Ag}\right)=-0.13056 \mu_{\mathrm{N}}: \mu_{\mathrm{I}}\left({ }^{107} \mathrm{Ag}\right)=$ $-0.11357 \mu_{\mathrm{N}}{ }^{36}$ Typically, however, in complexes such as $\left[\mathrm{Ag}\left(\mathrm{PPh}_{3}\right)_{n}\right]^{+}$ ( $n=1-4$ ) the solution exchange is rapid at room temperature so the doublet resonances are not resolved; they only become resolved at low temperature (below $203 \mathrm{~K}$ ). ${ }^{35 b}$ The ${ }^{31} \mathrm{P}$ NMR spectra of $\mathbf{1}$ and $\mathbf{2}$ in $\mathrm{CD}_{3} \mathrm{CN}$ are remarkable in that the doublet resonances are resolved at room temperature, indicative of slow exchange on the NMR time scale. In fact, resolution of the two doublets for ${ }^{109 / 107} \mathrm{Ag}-\mathrm{P}$ coupling is lost due to exchange broadening only after heating $\mathrm{CD}_{3} \mathrm{CN}$ solutions of $\mathbf{1}$ to 
$60^{\circ} \mathrm{C}$ or of 2 to $40^{\circ} \mathrm{C}$. At room temperature, the ${ }^{1} J_{107 \mathrm{Ag}-\mathrm{P}}$ coupling constant of $638 \mathrm{~Hz}$ for $\mathbf{1}$ and $644 \mathrm{~Hz}$ for $\mathbf{2}$, is comparable to other silver complexes with only one phosphine bound to silver such as $\left(\mathrm{Ph}_{3} \mathrm{P}\right) \mathrm{Ag}\left(\mathrm{NO}_{3}\right)(780 \mathrm{~Hz})$, ${ }^{30 a}\left(\mathrm{Ph}_{3} \mathrm{P}\right) \mathrm{Ag}\left(\mathrm{PF}_{6}\right)(755 \mathrm{~Hz})$, ${ }^{35 \mathrm{~b}}$ and $\left(\mathrm{tBu}_{3} \mathrm{P}\right) \mathrm{Ag}\left(\mathrm{NO}_{3}\right)(683 \mathrm{~Hz})$, ${ }^{35 \mathrm{~d}}$ or to other complexes binding one phosphine and pyrazolyl donors such as $\left[\left(\mathrm{Ph}_{3} \mathrm{P}\right) \mathrm{Ag}\left(\mathrm{pz}_{6} \mathrm{C}_{6}\right)\right]\left(\mathrm{SbF}_{6}\right)(648$ $\mathrm{Hz}),{ }^{37}\left[\left(\mathrm{n}^{5}-\mathrm{C}_{5} \mathrm{Me}_{5}\right) 1 \mathrm{r}(\mathrm{pz})_{3} \mathrm{Ag}\left(\mathrm{PPh}_{3}\right)\right](565 \mathrm{~Hz}){ }^{38}$ or $\left[\mathrm{HB}(\mathrm{pz})_{3}\right] \mathrm{Ag}\left(\mathrm{PPh}_{3}\right)$ $(607 \mathrm{~Hz}) .{ }^{39}$ Silver complexes bound to two phosphines have much smaller ${ }^{1} J 107_{\mathrm{Ag}-\mathrm{P}}$ coupling constants in the $400-500 \mathrm{~Hz}$ range such as $496 \mathrm{~Hz}$ for $\left[\left(p \text {-tolyl }{ }_{3} \mathrm{P}\right)_{2} \mathrm{Ag}\right] \mathrm{PF}_{6},{ }^{35 b} 507 \mathrm{~Hz}$ for $\left[\left(\mathrm{Ph}_{3} \mathrm{P}\right)_{2} \mathrm{Ag}\right]\left(\mathrm{PF}_{6}\right),{ }^{35 \mathrm{~b}}$ and $432 \mathrm{~Hz}$ for $\left[\left(\mathrm{tBu}_{3} \mathrm{P}\right)_{2} \mathrm{Ag}\right]\left(\mathrm{NO}_{3}\right) .{ }^{35 \mathrm{~d}}$ Thus, neither $\mathbf{1}$ nor $\mathbf{2}$ isomerize in solution to give a species where one silver is bound to two phosphines and the other silver is only bound to pyrazolyls (like Figure 1e). A second line of evidence that a cyclic structure is preserved in solution at room temperature comes from the ${ }^{1} \mathrm{H}$ NMR data of $\mathbf{2}$. The downfield portion of the spectrum and labeling scheme are shown on the right of Figure 8. Although we verified assignments of hydrogen resonances in $\boldsymbol{m} \boldsymbol{L}$ and $\mathbf{2}$ by 2D NMR (NOESY, COSY) experiments, the aryl hydrogen resonances (both the tolyl and central phenylene) of $\mathbf{2}$ can be assigned by simple inspection by considering relative integration, the asymmetric nature of the ligand that gives different distinctive multiplicities to the central phenylene resonances $\left(H_{\mathrm{e}-\mathrm{h}}\right.$, Figure 8$)$, and by the different magnitudes of the hydrogen-phosphorus coupling constants. The doublet resonance at $5.6 \mathrm{ppm}\left({ }^{3} \mathrm{~J}_{\mathrm{H}-\mathrm{P}}=9.5 \mathrm{~Hz}\right)$ for the single hydrogen atom, $H_{\mathrm{e}}$, situated between the phosphorus and the methine carbon, is highly shielded compared to other resonances or to that of the free ligand at $6.8 \mathrm{ppm}\left({ }^{3} \mathrm{~J}_{\mathrm{H}-\mathrm{P}}=7.1 \mathrm{~Hz}\right)$ in the same solvent. Inspection of the solid state structure of $\mathbf{2}$ (Figure $3 \mathrm{~b}$ ) shows that this $H_{\mathrm{e}}$ hydrogen is sandwiched between the $\mathrm{n}$-clouds of both a tolyl group and a pyrazolyl ring. Upon warming the solution to $60^{\circ} \mathrm{C}$, the doublet resonance for $H_{\mathrm{e}}$ becomes deshielded and exhibits the greatest downfield shift (of $0.2 \mathrm{ppm}$ ) compared with other resonances. The resonance for the tolyl hydrogens, $H_{i}$, close to the phosphorus atom, experiences the next greatest shift of $0.1 \mathrm{ppm}$ downfield, followed by the resonance for $H_{\mathrm{f}}$ (the other phenylene hydrogen ortho- to $\mathrm{P}$ ) which shifts upfield by $0.06 \mathrm{ppm}$. The resonances for pyrazolyl hydrogens only shift by $0.01 \mathrm{ppm}$ over the same temperature range. Thus, the environment around the phosphorus atom exhibits the greatest change with temperature, perhaps being indicative of dissociation at permission has been granted for this version to appear in e-Publications@Marquette. American Chemical Society does not grant permission for this article to be further copied/distributed or hosted elsewhere without the express permission from American Chemical Society. 
high temperature. Interestingly, there is only one set of resonances for each pyrazolyl and tolyl group in the ${ }^{1} \mathrm{H}$ NMR spectrum of either $\mathbf{1}$ or 2, even at low temperature $\left(233 \mathrm{~K}\right.$ for $\mathrm{CD}_{3} \mathrm{CN}, 193 \mathrm{~K}$ in acetone- $\left.d_{6}\right)$. Two sets of resonances for tolyl group hydrogens and two sets of resonances for pyrazolyl hydrogens were expected based on the solid state structures of the complexes that showed distinct "axial" and "equatorial" rings of each type. Thus, while the low temperature ${ }^{31} \mathrm{P}$ NMR data (and ${ }^{1} \mathrm{H}$ NMR data for $\mathbf{2}$ ) indicate that the metallacycles are intact, the 18- and 16-member metallacycles of $\mathbf{1}$ and $\mathbf{2}$, respectively, must have low barriers to inversion that would allow facile exchange axial and equatorial pyrazolyl or tolyl rings. Reger and co-workers have recently demonstrated similar dynamic behavior in metallacycles supported by related $m-\mathrm{pz}_{4} \times \mathrm{Xyl}^{7 \mathrm{~b}, 7 \mathrm{e}}$

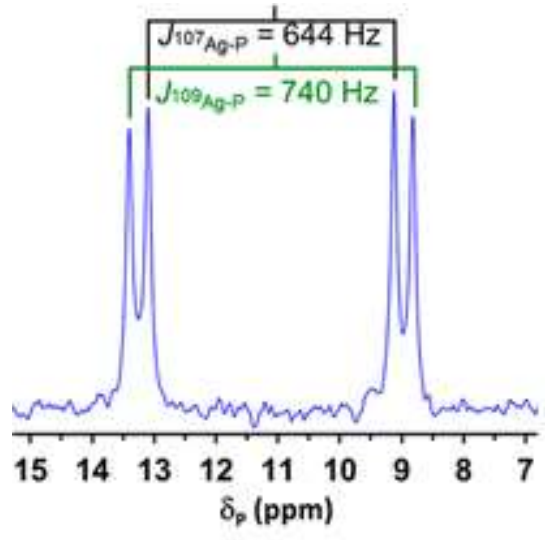

(a)

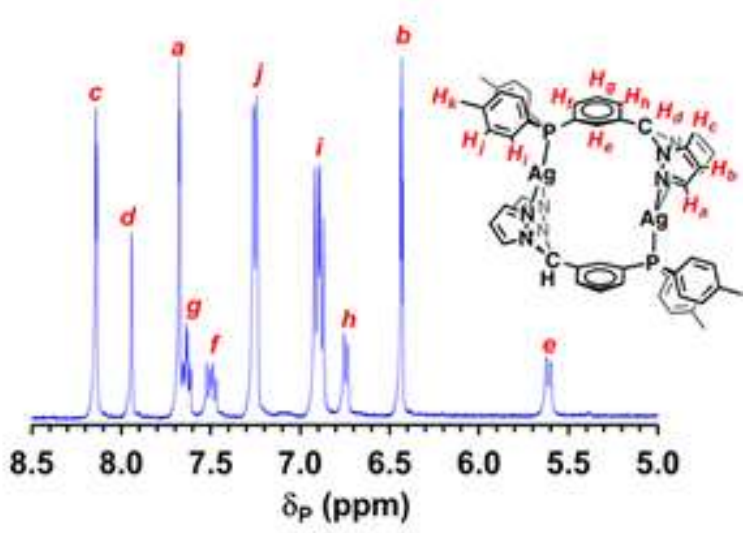

(b)

Figure 8. (a) ${ }^{31} \mathrm{P}$ NMR spectrum of $[\mathrm{Ag}(\boldsymbol{m L})](\mathrm{OTf}), \mathbf{2}$, in $\mathrm{CD}_{3} \mathrm{CN}$ at $295 \mathrm{~K}$ and (b) downfield portion of the ${ }^{1} \mathrm{H}$ NMR spectrum of $\mathbf{2}$ with atom labeling scheme.

In contrast to the above, the ${ }^{31} \mathrm{P}$ NMR spectrum of $\mathbf{3}$ in $\mathrm{CD}_{3} \mathrm{CN}$ shows only a broad singlet at room temperature due to (intermediate) exchange. Upon lowering the temperature to $273 \mathrm{~K}$, the coalescence temperature is reached, and the resonance appears as a broad doublet. Two doublet resonances for ${ }^{109} \mathrm{Ag}-\mathrm{P}$ and ${ }^{107} \mathrm{Ag}-\mathrm{P}$ coupling are only partially resolved at $233 \mathrm{~K}$, near the freezing point of the solvent, where the ${ }^{1} J_{107 \mathrm{Ag}-\mathrm{P}}$ coupling constant of $\mathrm{ca} .580 \mathrm{~Hz}$ is in line with other complexes with one phosphine bound to silver, as described above. It is of interest that the energy barrier to exchange (indicated by loss of Ag-P coupling) decreases with increasing steric bulk around the phosphorus atom of the ligand in the complexes in the order: $\mathbf{3}<\mathbf{2}<$ 
1. Such a trend further implicates that exchange occurs by dissociation of the phosphine.

Finally, the ${ }^{31} \mathrm{P}$ NMR spectrum of $\mathbf{4}$ in acetone- $d_{6}$ (Figure 9) at room temperature consists of two broad singlet resonances near $\delta_{p}$ $+11 \mathrm{ppm}$ and $-3 \mathrm{ppm}$, for the phosphorus nuclei of the $\mathrm{PPh}_{3}$ ligand and the $\mathrm{P}(p \text {-tolyl })_{2}$ moiety, respectively. Such an assignment is based on the observation that the difference between the chemical shift of the resonance for a silver(I) triarylphosphine complex and that for the free triarylphosphine ligand, $\Delta \delta_{\mathrm{P}}=\delta_{\mathrm{P}}($ complex $)-\delta_{\mathrm{P}}($ ligand $)$, is typically on the order of $+20 \mathrm{ppm} .{ }^{39}$ In this case, free $\mathrm{PPh}_{3}$ and the free ligand resonate at $\delta_{p}-6$ and $-20 \mathrm{ppm}$, respectively, giving $\Delta \delta_{\mathrm{p}}$ of $17 \mathrm{ppm}$ for each type of phosphine. For reference, the $\Delta \delta_{\mathrm{p}}$ for 1-3 were 19,18 , and $19 \mathrm{ppm}$, respectively. At low temperature, each singlet resonance of $\mathbf{4}$ resolves into two overlapping doublet of doublet resonances due to ${ }^{109} \mathrm{Ag}-\mathrm{P},{ }^{107} \mathrm{Ag}-\mathrm{P}$, and $\mathrm{P}-\mathrm{P}$ coupling as illustrated in the top of Figure 9. The ${ }^{1} J_{107 \mathrm{Ag}-\mathrm{P}}$ coupling constant of $484 \mathrm{~Hz}$ for the $\mathrm{PPh}_{3}$ moiety and of $396 \mathrm{~Hz}$ for the $\mathrm{P}(p \text {-tolyl })_{2}$ moiety are in the expected $400-500 \mathrm{~Hz}$ range for bis(triaryl)phosphine complexes, as outlined earlier. Moreover, the two different types of phosphorus lead to a ${ }^{2} J_{\mathrm{p}-\mathrm{P}}$ coupling of $108 \mathrm{~Hz}$, which is larger than $26 \mathrm{~Hz}$ found in $\left[\mathrm{PhB}\left(\mathrm{CH}_{2} \mathrm{PPh}_{2}\right)_{3}\right] \mathrm{Ag}\left(\mathrm{PEt}_{3}\right)^{40}$ but is on par with $134 \mathrm{~Hz}$ found for $\left\{\left[\mu-\left(\eta^{5}-\right.\right.\right.$ $\left.\left.\left.\mathrm{C}_{5} \mathrm{H}_{4}\right) \mathrm{PPh}_{2}\right] \mathrm{Ag}\left(\mathrm{PPh}_{3}\right)\right\}_{2 .}{ }^{41}$ At intermediary temperatures (213 $\mathrm{K}$ to ca. $253 \mathrm{~K}$ ) other resonances are observed in the baseline of the ${ }^{31} \mathrm{P} \mathrm{NMR}$ spectrum (between 10 to $5 \mathrm{ppm}$ and near $0 \mathrm{ppm}$ ), but their low intensity and the low signal-to-noise ratio prevented definitive assignment of these presumed intermediates or equilibrium species. 
NOT THE PUBLISHED VERSION; this is the author's final, peer-reviewed manuscript. The published version may be accessed by following the link in the citation at the bottom of the page.
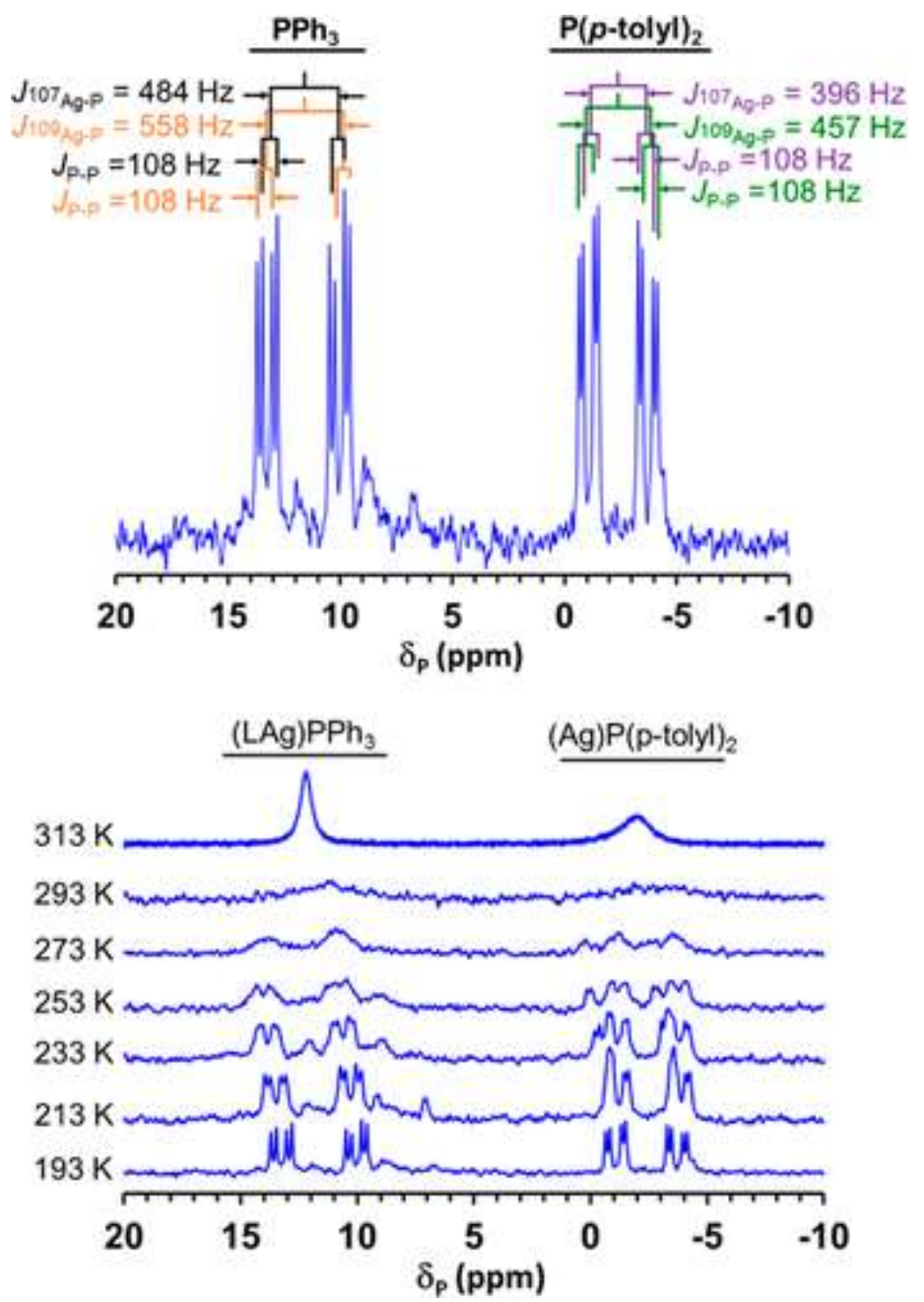

Figure 9. Top: ${ }^{31} \mathrm{P}$ NMR spectrum of $\left[\mathrm{Ag}(\mathbf{o L})\left(\mathrm{PPh}_{3}\right)\right](\mathrm{OTf}), \mathbf{4}$, in acetone- $d_{6}$ at $193 \mathrm{~K}$. Bottom: overlay of spectra of $\mathbf{4}$ in acetone- $d_{6}$ acquired at different temperatures.

\section{ESI(+) Mass Spectrometry}

Complexes 1-3 as $\mathrm{CH}_{3} \mathrm{CN}$ solutions were characterized by $\mathrm{ESI}(+)$ mass spectrometry. The parent (100\%) peak for $\mathbf{1}$ occurred at $m / z=544$ for the $\left[\mathrm{Ag}_{2} \mathrm{~L}_{2}\right]^{2+}$ dication. The identity of the dication versus an $[\mathrm{AgL}]^{+}$cation is easily determined by examination of the isotope pattern, especially since the former shows half-integer peaks, as in the right of Figure 10. Careful inspection of the intensities of the experimental data for the peaks near $m / z=544$ of 2 (most notably by the peaks at $m / z=543$ and 547 , right of Figure 10) reveals that they deviate slightly from the theoretical values for $\left[\mathrm{Ag}_{2} \mathrm{~L}_{2}\right]^{2+}$ because about $5-10 \%$ of the total signal in this region is for $[\mathrm{Ag}(\boldsymbol{m L})]^{+}$. The spectra 
of 1 and 2 also show a low abundance peak ( $1-2 \%$ ) at $m / z=1237$ for $\left[\mathrm{Ag}_{2} \mathrm{~L}_{2}(\mathrm{OTf})\right]^{+}$, providing further evidence for the persistence of the metallacycles under these conditions. In the spectrum of $\mathbf{3}$, the parent peak is at $m / z=979$ for $\left[\mathrm{AgL}_{2}\right]^{+}$. The second most abundant peak occurs at $m / z=584$ for $\left[(\boldsymbol{O L}) \mathrm{Ag}\left(\mathrm{CH}_{3} \mathrm{CN}\right)\right]^{+}$. A low abundance $(5 \%)$ peak found at $m / z=543$ is for the monocation $[\mathrm{AgL}]^{+}$. Collectively, the data indicate that $\mathbf{3}$ is extensively dissociated under these conditions rather than being polymeric akin to the complexes' solid state structure.
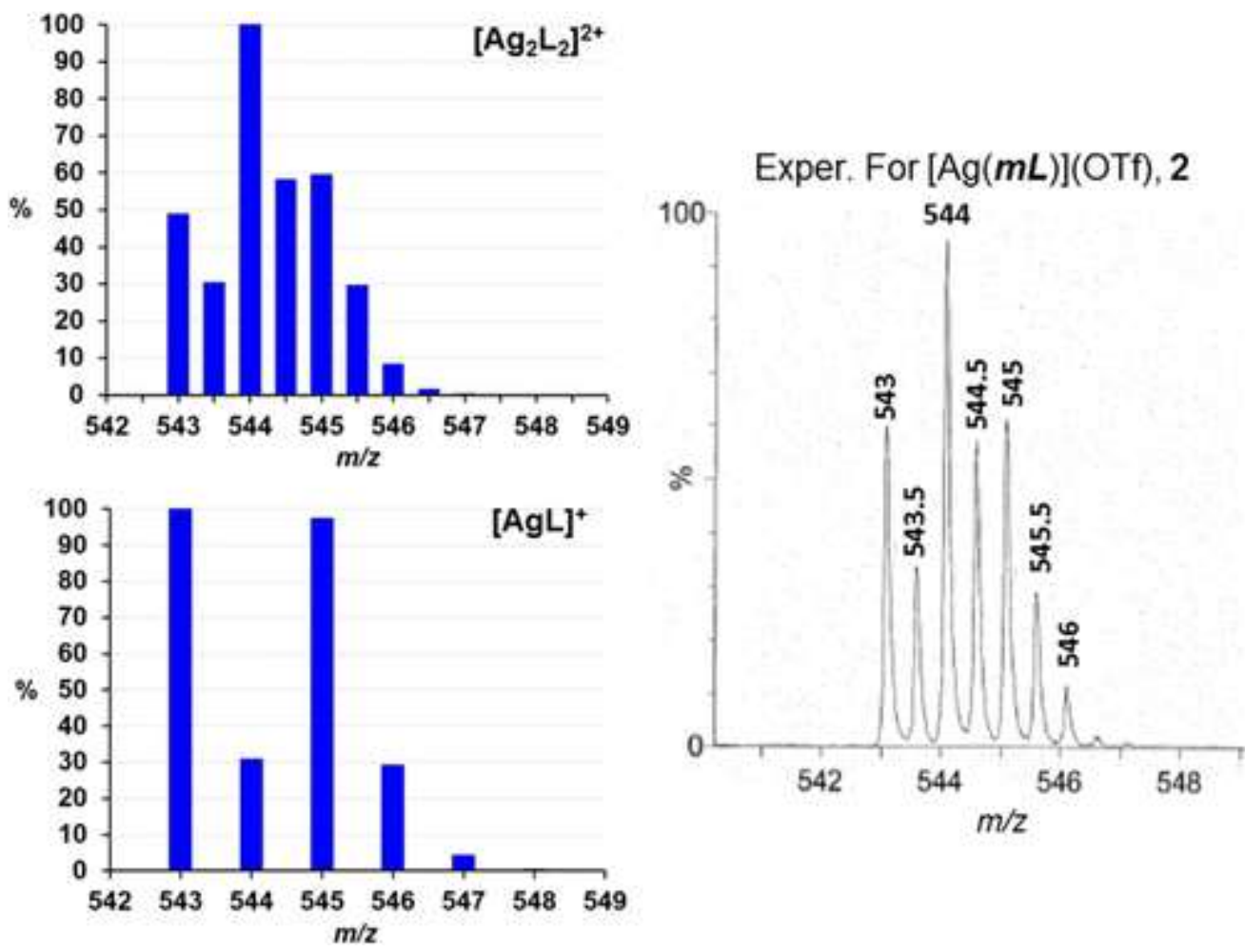

Figure 10. Comparison of theoretical isotope pattern for $[\mathrm{AgL}]^{+},\left[\mathrm{Ag}_{2} \mathrm{~L}_{2}\right]^{2+}$, and the experimental pattern for $\mathbf{2}$.

\section{Summary and Conclusions}

Silver(I) trifluoromethanesulfonate complexes of the newly prepared heteroditopic ligands containing di( $p$-tolyl)phosphine and di(pyrazolyl)methane groups bound to a phenylene spacer give different solid state and solution structures depending on the juxtaposition of the donor moieties. A coordination polymer, $\mathbf{3}$, is formed in the solid state if the donors are situated ortho- to one 
another on the phenylene ring. However, when the groups are disposed either para- (as in $\mathbf{1}$ ) or meta- (as in $\mathbf{2}$ ), the resultant silver complexes have cyclic bimetallic dications with 18- and 16-membered metallacyclic rings, respectively. In crystals of 1-0.5 acetone, 2. $\mathrm{CH}_{3} \mathrm{CN}$, and 3, the supramolecular structure was organized mainly by $\mathrm{CH} \cdots \mathrm{O}$ interactions involving the triflate oxygen and the acidic methine and 5-pyrazolyl hydrogens. The persistence of the cyclic structures of each $\mathbf{1}$ and $\mathbf{2}$ in $\mathrm{CH}_{3} \mathrm{CN}$ is evident by the $\sim 640 \mathrm{~Hz}$ onebond $\mathrm{Ag}-\mathrm{P}$ coupling in the room-temperature ${ }^{31} \mathrm{P} N \mathrm{NR}$ spectrum, the variable temperature ${ }^{1} \mathrm{H}$ NMR spectrum of $\mathbf{2}$, and the ESI $(+)$ mass spectrum of $\mathbf{1}$ and $\mathbf{2}$. The ${ }^{31} \mathrm{P}$ NMR data for $\mathbf{1}$ and $\mathbf{2}$ are particularly remarkable because one-bond $\mathrm{Ag}-\mathrm{P}$ coupling is not observed in the room temperature spectrum of $3,\left[\mathrm{Ag}\left(\mathrm{PR}_{3}\right)_{n}\right]^{+}(n=1-4)$, or most other heteroleptic [LAgP $]^{n+}$ complexes due to the lability of the Ag-P bond or bonds between other donors and silver. Evidently cyclic structures slow the rate of exchange, an observation that may have use in the future design of shape-persistent supramolecular assemblies of silver(I). The combination of NMR and ESI $(+)$ mass spectral data for 3 suggests that the coordination polymer is likely extensively dissociated in $\mathrm{CH}_{3} \mathrm{CN}$. Insight into the possible solution structure of $\mathbf{3}$ in $\mathrm{CH}_{3} \mathrm{CN}$ was gleaned by exploring the reaction between 3 and the strong Lewis donor $\mathrm{PPh}_{3}$, which gave a monomeric Lewis adduct $\mathbf{4}$ that showed a chelating $\mathrm{K}^{2}-P, N$ ligand in the solid state. The solution structure of $\mathbf{4}$ was dynamic where exchange involving phosphine could only be stopped at very low temperature (193 K in acetone- $\left.d_{6}\right)$. Future studies on the reactivity of these ligands and their silver(I) complexes toward transition metal salts is underway in our laboratory.

The authors declare no competing financial interest.

\section{References}

${ }^{1}$ (a) Foo, M. L.; Matsuda, R.; Kitagawa, S. Chem. Mater. 2014, 26, 310- 322. (b) Li, J.-R.; Kuppler, R. J.; Zhou, H.-C. Chem. Soc. Rev. 2009, 38, 1477-1504. (c) Férey, G. Chem. Soc. Rev. 2008, 37, 191- 214. (d) Maji, T. K.; Kitagawa, S. Pure Appl. Chem. 2007, 79, 2155- 2177. (e) Lee, J. Y.; Olson, D. H.; Pan, L.; Emge, T. J.; Li, J. Adv. Funct. Mater. 2007, 17, 1255- 1262

${ }^{2}$ (a) Wei, L.; Wei, Q.; Lin, Z.-E.; Meng, Q.; He, H.; Yang, B.-F.; Yang, G.-Y. Angew. Chem., Int. Ed. 2014, 53, 7188- 7191. (b) Heine, J.; MuellerBuschbaum, K. Chem. Soc. Rev. 2013, 42, 9232- 9242. (c) Xu, C.; 
NOT THE PUBLISHED VERSION; this is the author's final, peer-reviewed manuscript. The published version may be accessed by following the link in the citation at the bottom of the page.

Zhang, Z.-Y.; Ren, Z.-G.; Zhou, L.-K.; Li, H.-X.; Wang, H.-F.; Sun, Z.R.; Lang, J.-P. Cryst. Growth Des. 2013, 13, 2530- 2539. (d) Cui, Y.; Yue, Y.; Chen, B. Chem. Rev. 2012, 112, 1126- 1162

3(a) Butler, K. T.; Hendon, C. H.; Walsh, A. J. Am. Chem. Soc. 2014, 136, 2703- 2706. (b) Nafady, A.; O'Mullane, A. P.; Bond, A. M. Coord. Chem. Rev. 2014, 268, 101- 142. (c) Givaja, G.; Amo-Ochoa, P.; Gomez-Garcia, C. J.; Zamora, F. Chem. Soc. Rev. 2012, 41, 115- 147.

(d) Gomez-Herrero, J.; Zamora, F. Adv. Mater. 2011, 23, 5311- 5317.

(e) Zhang, W.; Ye, H.-Y.; Xiong, R.-G. Coord. Chem. Rev. 2009, 253, 2980- 2997. (f) Janiak, C. Dalton Trans. 2003, 14, 2781- 2804. (g) Chen, C. T.; Suslick, K. S. Coord. Chem. Rev. 1993, 128, 293- 322

${ }^{4}$ (a) Wang, L.; Wang, W.; Di, S.; Yang, X.; Chen, H.; Gong, T.; Zhou, S. RSC Adv. 2014. (b) Tan, H.; Zhang, L.; Ma, C.; Song, Y.; Xu, F.; Chen, S.; Wang, L. ACS Appl. Mater. Interfaces 2013, 5, 11791- 11796. (c) Lu, X.; Cheng, H.; Huang, P.; Yang, L.; Yu, P.; Mao, L. Anal. Chem. 2013, 85, 4007- 4013. (d) Novio, F.; Simmchen, J.; Vazquez-Mera, N.; Amorin-Ferre, L.; Ruiz-Molina, D. Coord. Chem. Rev. 2013, 257, 2839- 2847. (e) Tabacaru, A.; Pettinari, C.; Marchetti, F.; di Nicola, C.; Domasevitch, K. V.; Galli, S.; Masciocchi, N.; Scuri, S.; Grappasonni, I.; Cocchioni, M. Inorg. Chem. 2012, 51, 9775- 9788

${ }^{5}$ (a) Li, L.; Matsuda, R.; Tanaka, I.; Sato, H.; Kanoo, P.; Jeon, H. J.; Foo, M. L.; Wakamiya, A.; Murata, Y.; Kitagawa, S. J. Am. Chem. Soc. 2014, 136, 7543- 7546. (b) Cook, T. R.; Zheng, Y.-R.; Stang, P. J. Chem. Rev. 2013, 113, 734- 777. (c) Wang, C.; Liu, D.; Lin, W. J. Am. Chem. Soc. 2013, 135, 13222- 13234. (d) Zhao, D.; Timmons, D. J.; Yuan, D.; Zhou, H. C. Acc. Chem. Res. 2011, 44, 123- 133. (e) Ranocchiari, M.; van Bokhoven, J. A. Phys. Chem. Chem. Phys. 2011, 13, 6388- 6396. (f) Uemura, T.; Yanai, N.; Kitagawa, S. Chem. Soc. Rev. 2009, 38, 1228- 1236. (g) Kitagawa, S.; Kitaura, R.; Noro, S.-i. Angew. Chem., Int. Ed. 2004, 43, 2334- 2375

${ }^{6}$ (a) Mukherjee, A.; Tothadi, S.; Desiraju, G. R. Acc. Chem. Res. 2014. (b) Bombicz, P.; Gruber, T.; Fischer, C.; Weber, E.; Kalman, A. CrystEngComm 2014, 16, 3646- 3654. (c) Cherukuvada, S.; Guru Row, T. N. Cryst. Growth Des. 2014, 14. (d) Cavallo, G.; Metrangolo, P.; Pilati, T.; Resnati, G.; Terraneo, G. Cryst. Growth Des. 2014, 14, 2697- 2702. (e) Goesten, M. G.; Kapteijn, F.; Gascon, J.

CrystEngComm 2013, 15, 9249- 9257. (f) Mastalerz, M. Nat. Chem. 2013, 5, 810-811. (g) Siddiqui, K. A.; Tiekink, E. R. T. Chem. Commun. 2013, 49, 8501- 8503. (h) Desiraju, G. R. J. Am. Chem. Soc. 2013, 135, 9952- 9967. (i) Du, M.; Li, C.-P.; Liu, C.-S.; Fang, S.M. Coord. Chem. Rev. 2013, 257, 1282- 1305. (j) Aakeröy, C. B.; Panikkattu, S. V.; DeHaven, B.; Desper, J. Cryst. Growth Des. 2012, 12, 2579- 2587. (k) Aakeröy, C. B.; Champness, N.; Janiak, C. 
NOT THE PUBLISHED VERSION; this is the author's final, peer-reviewed manuscript. The published version may be accessed by following the link in the citation at the bottom of the page.

CrystEngComm 2010, 12, 22- 43. (I) Braga, D. Chem. Commun. 2003, 2751- 2754. (m) Aakeröy, C. B. Acta Crystallogr., Sect. B: Struct. Sci. 1997, 53, 569- 586. (n) Desiraju, G. R. Angew. Chem., Int. Ed. 1995, 34, 2311- 2327

${ }^{7}$ (a) Reger, D. L.; Watson, R. P.; Smith, M. D. Inorg. Chem. 2006, 45, 1007710087. (b) Reger, D. L.; Pascui, A. E.; Pellechia, P. J.; Smith, M. D.; Jezierska, J.; Ozarowski, A. Inorg. Chem. 2014, 53, 4325-4339. (c) Reger, D. L.; Pascui, A. E.; Foley, E. A.; Smith, M. D.; Jezierska, J.; Ozarowski, A. Inorg. Chem. 2014, 53, 1975- 1988. (d) Reger, D. L.; Pascui, A. E.; Pellechia, P. J.; Ozarowski, A. Inorg. Chem. 2013, 52, 12741- 12748. (e) Reger, D. L.; Pascui, A. E.; Pellechia, P. J.; Smith, M. D. Inorg. Chem. 2013, 52, 11638-11649. (f) Reger, D. L.; Pascui, A. E.; Smith, M. D.; Jezierska, J.; Ozarowski, A. Inorg. Chem. 2012, $51,11820-11836$

8(a) Gardinier, J. R.; Tatlock, H. M.; Hewage, J. S.; Lindeman, S. V. Cryst. Growth Des. 2013, 13, 3864- 3877. (b) Durá, G.; Carrión, M. C.; Jalón, F. A.; Manzano, B. R.; Rodríguez, A. M. Eur. J. Inorg. Chem. 2013, 5943- 5957

9(a) Durá, G.; Carrión, M. C.; Jalón, F. A.; Rodríguez, A. M.; Manzano, B. R. Cryst. Growth Des. 2014, 14, 3510- 3529. (b) Durá, G.; Carrión, M. C.; Jalón, F. A.; Rodríguez, A. M.; Manzano, B. R. Cryst. Growth Des. 2013, 13, 3275- 3282. (c) Carrión, M. C.; Durá, G.; Jalón, F. A.; Manzano, B. R.; Rodríguez, A. M. Cryst. Growth Des. 2012, 12, 19521969

${ }^{10}$ (a) Santillan, G. A.; Carrano, C. J. Dalton Trans. 2009, 6599- 6605. (b) Santillan, G. A. ; Carrano, C. J. Cryst. Growth Des. 2009, 9, 15901598. (c) Santillan, G. A.; Carrano, C. J. Inorg. Chem. 2008, 47, 930939. (d) Santillan, G. A.; Carrano, C. J. Dalton Trans. 2008, 39954005

${ }^{11}$ (a) Bassanetti, I.; Mezzadri, F.; Comotti, A. ; Sozzani, P.; Gennari, M. ; Calestani, G.; Marchiò, L. J. Am. Chem. Soc. 2012, 134, 9142- 9145. (b) Bassanetti, I.; Marchiò, L. Inorg. Chem. 2011, 50, 10786- 10797

${ }^{12}$ (a) Reger, D. L.; Watson, R. P.; Gardinier, J. R.; Smith, M. D. Inorg. Chem. 2004, 43, 6609- 6619. (b) Reger, D. L.; Gardinier, J. R.; Grattan, T. C.; Smith, M. R.; Smith, M. D. New J. Chem. 2003, 27, 1670- 1677. (c) Reger, D. L.; Brown, K. J.; Gardinier, J. R.; Smith, M. D. Organometallics 2003, 22, 4973-4983. (d) Reger, D. L.; Gardinier, J. R.; Semeniuc, R. F.; Smith, M. D. Dalton Trans. 2003, 9, 1712- 1718

${ }^{13}$ Li, Q.; Xie, Y.-F.; Sun, B.-C.; Yang, J.; Song, H.-B.; Tang, L.-F. J. Organomet. Chem. 2013, 745-746, 106- 114

${ }^{14}$ (a) Bassanetti, I.; Gennari, M.; Marchiò, L.; Terenghi, M.; Elviri, L. Inorg. Chem. 2010, 49, 7007- 7015. (b) Gennari, M.; Bassanetti, I.; Marchiò, L. Polyhedron 2010, 29, 361- 371.

Inorganic Chemistry, Vol 53, No. 22 (2014): pg. 12108-12121. DOI. This article is @ American Chemical Society and permission has been granted for this version to appear in e-Publications@Marquette. American Chemical Society does not grant permission for this article to be further copied/distributed or hosted elsewhere without the express permission from American Chemical Society. 
NOT THE PUBLISHED VERSION; this is the author's final, peer-reviewed manuscript. The published version may be accessed by following the link in the citation at the bottom of the page.

${ }^{15}$ Morin, T. J.; Merkel, A.; Lindeman, S. V.; Gardinier, J. R. Inorg. Chem. 2010, 49, 7992- 8002

${ }^{16}$ (a) Bassanetti, I.; Marchiò, L. Inorg. Chem. 2011, 50, 10786- 10797. (b)

Ding, K.; Cheng, C.-H.; Yang, Y.-X.; Song, H.-B.; Tang, L.-F. J.

Organomet. Chem. 2011, 696, 3662- 3667. (c) Blasberg, F.; Bats, J. W.; Bolte, M.; Lerner, H.-W.; Wagner, M. Inorg. Chem. 2010, 49, 7435- 7445. (d) Peters, L.; Hübner, E.; Haas, T.; Heinemann, F. W.; Burzlaff, N. J. Organomet. Chem. 2009, 694, 2319- 2327. (a) Chandrasekhar, V.; Thilagar, P.; Senapati, T. Eur. J. Inorg. Chem. 2007, 1004- 1009. (f) Otero, A.; Fernandez-Baeza, J.; Antinolo, A. ; Carrillo-Hermosilla, F.; Tejeda, J.; Lara-Sanchez, A.; Sanchez-Barba, L.; Fernandez-Lopez, M.; Rodriguez, A. M.; Lopez-Solera, I. Inorg. Chem. 2002, 41, 5193- 5202

${ }^{17}$ Coulson, D. R.; Satek, L. C.; Grim, S. O. Inorg. Synth. 1990, 28, 107- 109

${ }^{18}$ CrysAlisPro, Agilent Technologies,Version 1.171.34.46 (release 25-11-2010 CrysAlis171.NET), (compiled Nov 25 2010, 17:55:46).

${ }^{19}$ Olex2 1.2 (compiled 2013.11.15 svn.r2834 for OlexSys, GUI svn.r4720). Dolomanov, O. V.; Bourhis, L. J.; Gildea, R. J.; Howard, J. A. K.; Puschmann, H. J. Appl. Crystallogr. 2009, 42, 339- 341

${ }^{20}$ Sheldrick, G. M. SHELXTL, Version 6.12; Bruker Analytical X-ray Systems, Inc.: Madison Wisconsin, USA, 2001.

${ }^{21}$ (a) Thé, K. I.; Peterson, L. K. Can. J. Chem. 1973, 51, 422- 426. (b) Thé, K. I.; Peterson, L. K.; Kiehlmann, E. Can. J. Chem. 1973, 51, 24482451. (c) Peterson, L. K.; Kiehlmann, E.; Sanger, A. R.; Thé, K. I. Can. J. Chem. 1974, 52, 2367- 2374

${ }^{22}$ Liddle, B. J.; Hall, D.; Lindeman, S. V.; Smith, M. D.; Gardinier, J. R. Inorg. Chem. 2009, 48, 8404- 8414

${ }^{23}$ Stein, R. A.; Knobler, C. Inorg. Chem. 1977, 16, 242- 245

${ }^{24}$ (a) Terrobaa, R.; Hursthouse, M. B.; Laguna, M.; Mendiac, A. Polyhedron 1999, 18, 807-810. (b) Bardají, M.; Crespo, O.; Laguna, A.; Fischer, A. K. Inorg. Chim. Acta 2000, 304, 7- 16

${ }^{25}$ Teo, B.-K.; Calabrese, J. C. Inorg. Chem. 1976, 15, 2467- 2474

${ }^{26}$ For example: Argyle, V. J.; Woods, L. M.; Roxburgh, M.; Hanton, L. R. CrystEngComm 2013, 15, 120- 134

${ }^{27}$ CSD Version 5.35, November 2013.

${ }^{28}$ Yang, L.; Powell, D. R.; Houser, R. P. Dalton Trans. 2007, 955- 964

${ }^{29}$ The closest $\mathrm{Ag} 1 \cdots \mathrm{O} 3$ distance in $2 \cdot \mathrm{CH}_{3} \mathrm{CN}$ is $3.796 \AA$; there is a longer Ag1 $\cdots$ F2 contact of $3.1068(19) \AA$ that is less than $3.24 \AA$, the sum of van der Waals radii, but this contact is best classified as a secondary interaction.

${ }^{30}$ See for example: (a) Barron, P. F.; Dyason, J. C.; Healy, P. C.; Engelhardt, L. M.; Skelton, B. W.; White, A. H. J. Chem. Soc., Dalton Trans. 1986, 1965- 1970. (b) Cingolani, A.; Effendy; Hanna, J. V.; Pellei, M.;

Inorganic Chemistry, Vol 53, No. 22 (2014): pg. 12108-12121. DOI. This article is @ American Chemical Society and permission has been granted for this version to appear in e-Publications@Marquette. American Chemical Society does not grant permission for this article to be further copied/distributed or hosted elsewhere without the express permission from American Chemical Society. 
Pettinari, C.; Santini, C.; Skelton, B. W.; White, A. H. Inorg. Chem. 2003, 42, 4938- 4948. (c) Bachman, R. E.; Andretta, D. F. Inorg. Chem. 1998, 37, 5657- 5663 and references

${ }^{31}$ (a) Zartilas, S.; Hadjikakou, S. K.; Hadjiliadis, N.; Kourkoumelis, N.; Kyros, L.; Kubicki, M.; Baril, M.; Butler, I. S.; Karkabounas, S.; Balzarini, J. Inorg. Chim. Acta 2009, 362, 1003- 1010. (b) Omondi, B.; Venter, G. J. S.; Roodt, A.; Meijboom, R. Acta Crystallogr. 2009, B65, 699- 706. (c) Venter, G. J. S.; Roodt, A.; Meijboom, R. Acta Crystallogr. 2009, B65, 182- 188. (e) Venter, G. J. S.; Meijboom, R.; Roodt, A. Acta Crystallogr., Sect. E 2006, 62, m3453- m3455. (f) Camalli, M.; Caruso, F. Inorg. Chim. Acta 1987, 127, 209- 213

${ }^{32}$ (a) Pellei, M.; Alidori, S.; Papini, G.; Lobbia, G. G.; Gorden, J. D.; Dias, H. V. R.; Santini, C. Dalton Trans. 2007, 42, 4845- 4853. (b) Dias, H. V. R.; Alidori, S.; Lobbia, G. G.; Papini, G.; Pellei, M.; Santini, C. Inorg. Chem. 2007, 46, 9708- 9714. (c) Santini, C.; Pellei, M.; Alidori, S.; Lobbia, G. G.; Benetollo, F. Inorg. Chim. Acta 2007, 360, 2121- 2127. (d) Pettinari, C.; Cingolani, A.; Lobbia, G. G.; Marchetti, F.; Martini, D.; Pellei, M.; Pettinari, R.; Santini, C. Polyhedron 2004, 23, $451-469$

${ }^{33}$ (a) Wheeler, S. E.; Bloom, J. W. G. J. Phys. Chem. A 2014. (b) Bernstein, J. Cryst. Growth Des. 2013, 13, 961-964. (c) Gu, Y.; Kar, T.; Scheiner, S. J. Am. Chem. Soc. 1999, 121, 9411-9422. (d) Desiraju, G. J. Acc. Chem. Res. 1996, 29, 441- 449. (e) Steiner, T.; Saenger, F. J. Am. Chem. Soc. 1992, 114, 10146- 10154. (f) Desiraju, G. J. Acc. Chem. Res. 1991, 24, 290- 296. (g) Taylor, R.; Kennard, O. J. Am. Chem. Soc. $1982,104,5063-5070$

${ }^{34}$ (a) Lawrance, G. A. Chem. Rev. 1986, 86, 17- 33. (b) Grochala, W.; Cyránski, M. K.; Derzsi, M.; Michałowski, T.; Malinowski, P. J.; Mazej, Z.; Kurzydłowski, D.; Kózmínski, W.; Budzianowskia, A.; Leszczýnski, P. J. Dalton Trans. 2012, 41, 2034- 2047

${ }^{35}$ (a) Meijboom, R.; Bowen, R. J.; Berners-Price, S. J. Coord. Chem. Rev. 2009, 253, 325- 342 and references. (b) Chen, F.; Oh, S.-W.; Wasylishen, R. E. Can. J. Chem. 2009, 87, 1090-1101. (c) Alyea, E. C.; Malito, J.; Nelson, J. H. Inorg. Chem. 1987, 26, 4294- 4296. (d) Goel, R. G.; Pilon, P. Inorg. Chem. 1978, 17, 2876- 2879. (e) Muetterties, E. L.; Alegranti, C. W. Inorg. Chem. 1972, 94, 63866391

${ }^{36}$ CRC Handbook of Chemistry and Physics, 95th ed.; Haynes, W. M., Ed. ; CRC Press: Boca Raton, FL, 2014-2015; Section 11, 55- 56; http://www.hbcpnetbase.com (accessed August 5, 2014).

${ }^{37}$ Caballero, A.; Guerrero, A.; Jalón, F. A.; Manzano, B. R.; Claramunt, R. M.; María, M. D. S.; Escolástico, C.; Elguero, J. Inorg. Chim. Acta 2003, $347,168-174$ 
NOT THE PUBLISHED VERSION; this is the author's final, peer-reviewed manuscript. The published version may be accessed by following the link in the citation at the bottom of the page.

${ }^{38}$ Carmona, D.; Oro, L. A.; Lamata, M. P.; Jimeno, M. L.; Elguero, J.;

Belguise, A.; Lux, P. Inorg. Chem. 1994, 33, 2196- 2203

${ }^{39}$ Santini, C.; Lobbia, G. G.; Pettinari, C.; Pellei, M.; Valle, G.; Calogero, S. Inorg. Chem. 1998, 37, 890-900

${ }^{40}$ McCain, M. N.; Schneider, S.; Salata, M. R.; Marks, T. J. Inorg. Chem. 2008, 47, 2534- 2542

${ }^{41}$ Lettko, L.; Rausch, M. D. Organometallics 2000, 19, 4060- 4065 


\section{Supporting Information for}

Isomer Dependence in the Assembly and Lability of Silver(I) Trifluoromethanesulfonate Complexes of the Heteroditopic Ligands 2-, 3-, and 4-[di(1H-pyrazolyl)methyl]phenyl(di-p-tolyl)phosphine.

James R. Gardinier*, Jeewantha S. Hewage, and Sergey V. Lindeman

\section{Table of Contents:}

Structure of $\left\{\left[\left(m-\mathrm{IC}_{6} \mathrm{H}_{4}\right) \mathrm{CHpz}_{2}\right]_{2} \mathrm{Ag}\right\}(\mathrm{OTf}), \mathbf{5}$.

Details and results of CSD Query of Ag-O(triflate) distances.

Supramolecular structure of $\mathbf{2} \cdot \mathrm{CH}_{3} \mathrm{CN}$.

Supramolecular structure of $[\mathrm{Ag}(\mathrm{oL})](\mathrm{OTf}), 3$.

Supramolecular structure of $\left[\left(m-\mathrm{IC}_{6} \mathrm{H}_{4} \mathrm{CHpz}_{2}\right)_{2} \mathrm{Ag}\right](\mathrm{OTf}), \mathbf{5}$.

Powder X-ray Diffraction patterns for 2, 3, and $\mathbf{5 .}$

IR spectra of 1-4 and 5.

Figures for NMR assignments and 2D NMR spectra 


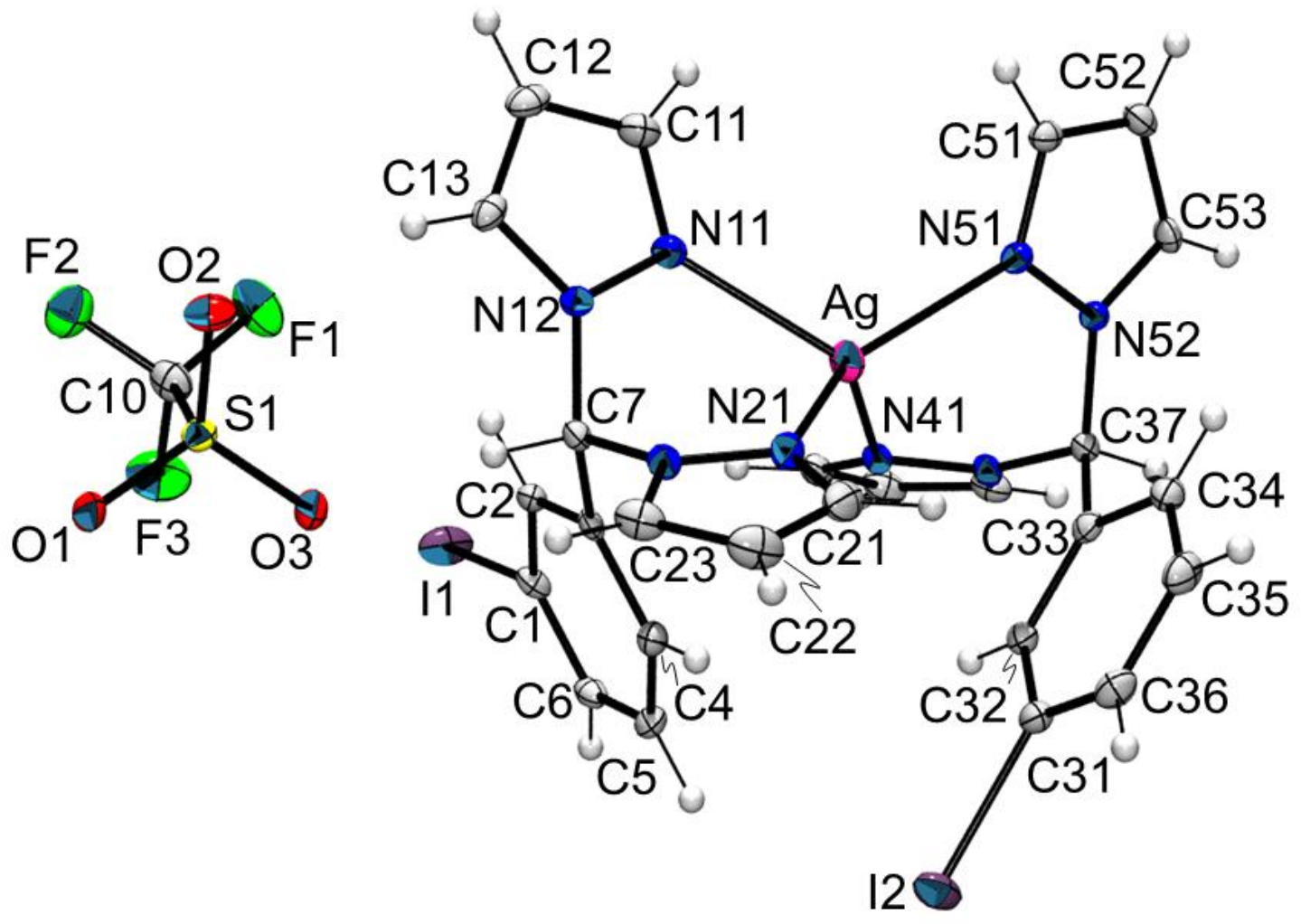

Figure S1. Structure of $\left[\left(m-\mathrm{IC}_{6} \mathrm{H}_{4} \mathrm{CHpz}_{2}\right)_{2} \mathrm{Ag}\right](\mathrm{OTf})$, 5. Selected bond distances $(\AA ̊): \operatorname{Ag} 1-\mathrm{N} 11$ 2.314(3), Ag1-N21 2.269(3), Ag1-N41 2.326(3), Ag1-N51 2.263(2). Selected bond angles ( $\left.{ }^{\circ}\right)$ : N11-Ag1-N41 113.21(9), N21-Ag1-N11 85.52(9), N21-Ag1-N41 128.40(9), N51-Ag1-N11 119.72(9), N51-Ag1-N21 128.05(9), N51-Ag1-N41 85.20(8). 
Details of Cambridge Structural data base (CSD) Search.

(a) $\mathrm{Ag} \cdots \mathrm{OSO}_{2} \mathrm{CF}_{3}$ interactions. The query shown in Scheme S1 drawn in the ConQuest version 1.15 software package ${ }^{(\mathrm{S} 1)}$ was used in the search of Cambridge Structural Database,<smiles>O[Si](O)(O[Ge])C(F)(F)F</smiles>

Scheme S1. Diagram used in query of CSD search.

version 5.35 (updates November 2013). The bonds between $\mathrm{Ag}$ and $\mathrm{O}$ as well as those between $\mathrm{S}$ and $\mathrm{O}$ were set to "any", indicated by the dashed lines in Scheme S1. Those bonds between S and $\mathrm{C}$ and between $\mathrm{C}$ and $\mathrm{F}$ were single. This search produced 432 "hits" (CSD refcodes) with a total of 1047 entries due to multiple entries (Ag-O interactions) per hit. A histogram showing the bond distance distribution of the 1047 entries is found in Figure S2. The minimum distance was $2.075 \AA$, the maximum was $3.017 \AA$, the mean was $2.48 \AA$, the median was $2.47 \AA$ and the standard deviation was $0.13 \AA$.

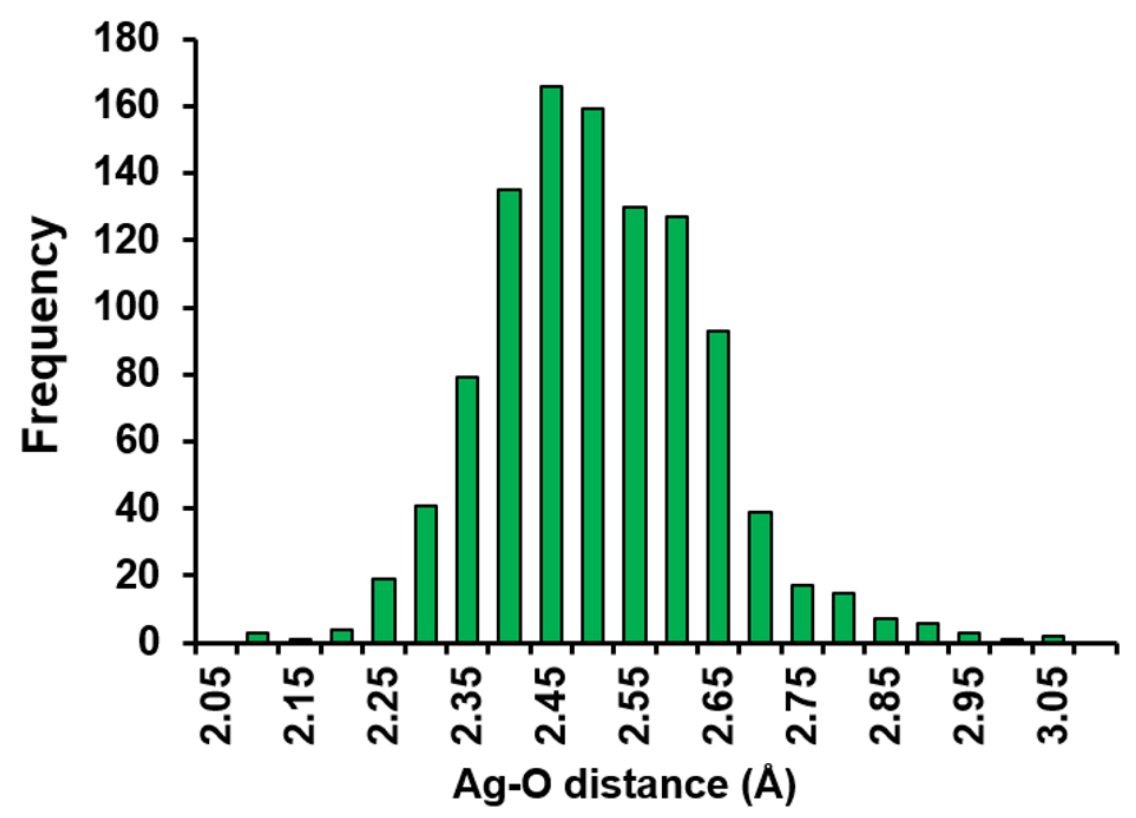

Figure S2. Histogram showing distribution of Ag-O distances (A). 


\section{Supramolecular Structures.}

$[\mathrm{Ag}(\boldsymbol{m L})](\mathrm{OTf}) \cdot \mathrm{CH}_{3} \mathrm{CN}, \mathbf{2} \cdot \mathrm{CH}_{3} \mathrm{CN}$. Figure $\mathrm{S} 3$ and Table $\mathrm{S} 1$ provide illustrations and metrics of the noncovalent interactions that organize $2 \cdot \mathrm{CH}_{3} \mathrm{CN}$ into a 3D layered sheet structure. The $\mathrm{CH} \cdots \mathrm{O}^{(\mathrm{s} 2)}$

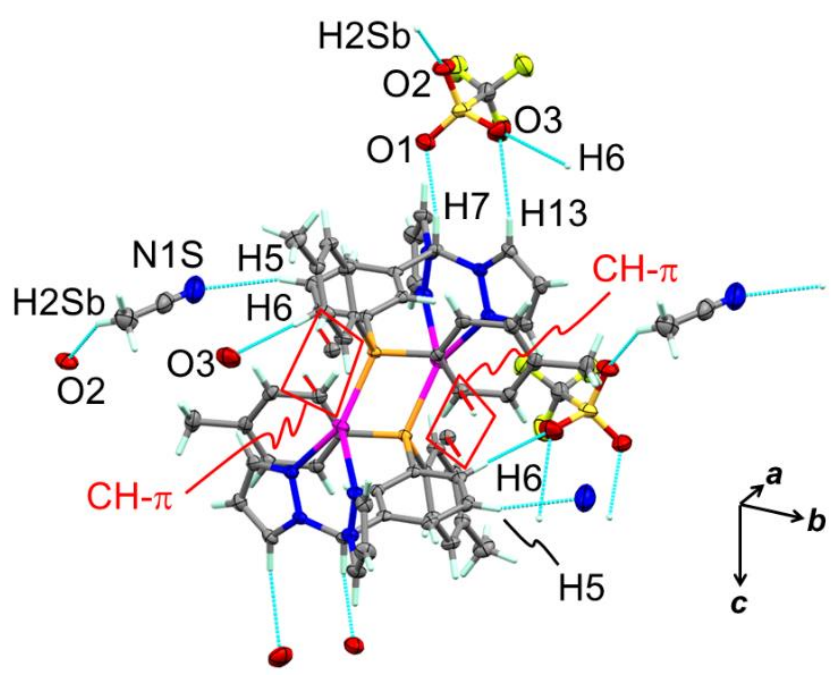

(a)

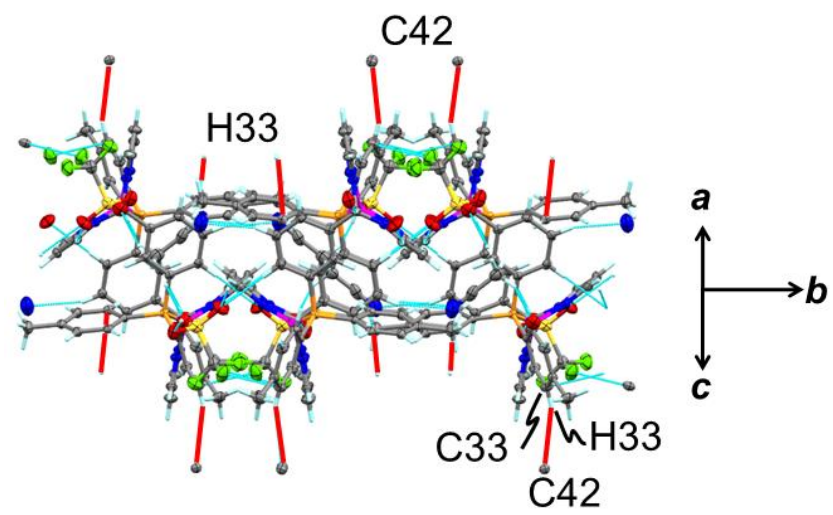

(c)

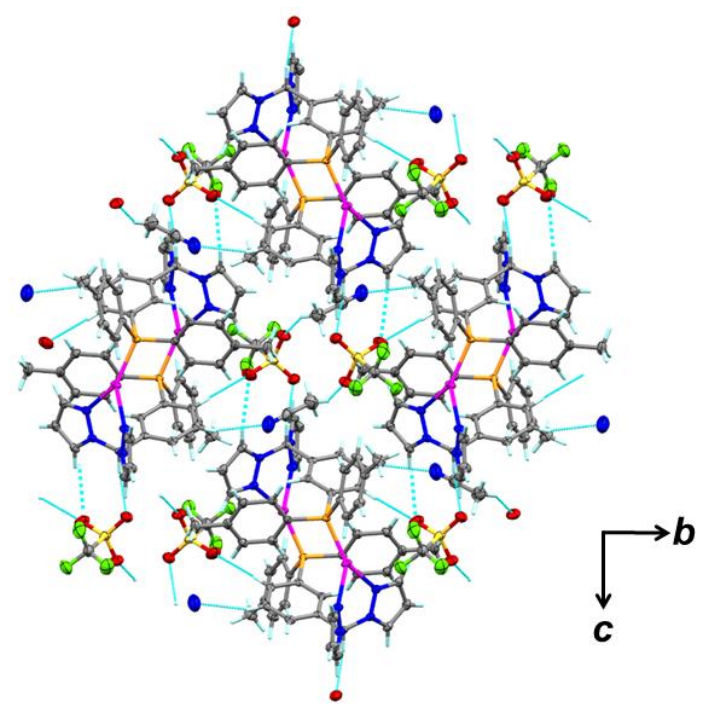

(b)

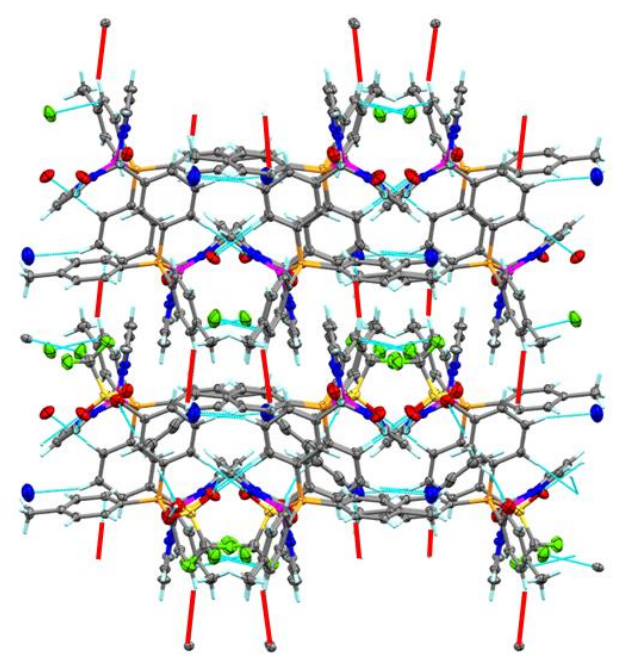

(d)

Figure S3. Supramolecular structure of $2 \cdot \mathrm{CH}_{3} \mathrm{CN}$. (a) view of $\mathrm{CH} \cdots \mathrm{O}$ interactions (cyan lines) and $\mathrm{CH} \cdots \pi$ interactions (red lines) that organize the structure and atom labeling. (b) View down $a$-axis of four 2. $\mathrm{CH}_{3} \mathrm{CN}$ units in a sheet showing only $\mathrm{CH} \cdots \mathrm{O}$ interactions. (c) Same four units but viewed perpendicular to $b$-axis and with $\mathrm{CH} \cdots \pi$ interactions (highlighted as red lines). (d) Two sheets stacked by $\mathrm{CH} \cdots \pi$ interactions. 
Table S1. Geometries of weak $\mathrm{C}-\mathrm{H} \cdots\left(\mathrm{X}=\mathrm{O}^{(\mathrm{s} 2)}, \mathrm{N}^{(\mathrm{s} 2 \mathrm{f}, \mathrm{s})}\right)$ and $\mathrm{C}-\mathrm{H} \cdots \pi^{(\mathrm{s} 4)}$ interactions in $[\mathrm{Ag}(\boldsymbol{m L})](\mathrm{OTf}) \mathrm{CH}_{3} \mathrm{CN}$, $2 \mathrm{CH}_{3} \mathrm{CN}$.

\begin{tabular}{|c|c|c|c|c|c|}
\hline Donor(D)(-H) $\cdots$ Acceptor(A) & $\mathrm{D}-\mathrm{H}(\AA)$ & $\mathrm{H} \cdots \mathrm{A}(\AA)$ & $D \cdots A(\AA)$ & $\mathrm{D}-\mathrm{H} \cdots \mathrm{A}\left({ }^{\circ}\right)$ & $\gamma\left({ }^{\circ}\right)$ \\
\hline \multicolumn{6}{|c|}{$\mathrm{C}-\mathrm{H} \cdots \times$ interactions } \\
\hline $\mathrm{C} 7-\mathrm{H} 7 \cdots \mathrm{O} 1$ & 1.00 & 2.16 & $3.126(3)$ & 163 & \\
\hline $\mathrm{C} 6-\mathrm{H} 6 \cdots \mathrm{O} 3$ & 0.95 & 2.50 & $3.452(3)$ & 177 & \\
\hline $\mathrm{C} 13-\mathrm{H} 13 \cdots \mathrm{O} 3$ & 0.95 & 2.59 & $3.474(3)$ & 155 & \\
\hline $\mathrm{C} 2 \mathrm{~S}-\mathrm{H} 2 \mathrm{Sb} \cdots \mathrm{O} 2$ & 0.98 & 2.37 & $3.231(4)$ & 147 & \\
\hline $\mathrm{C} 5-\mathrm{H} 5 \cdots \mathrm{N} 1 \mathrm{~s}$ & 0.95 & 2.54 & $3.439(4)$ & 159 & \\
\hline \multicolumn{6}{|c|}{$\mathrm{C}-\mathrm{H} \cdots \pi$ interaction } \\
\hline $\mathrm{C} 33-\mathrm{H} 33 \cdots \mathrm{Ct}(\mathrm{N} 41)$ & 0.95 & 2.56 & $3.502(3)$ & 170 & 5.4 \\
\hline
\end{tabular}

Interactions between the triflate oxygen atoms and acidic hydrogens of the dication serve to assemble sheets in the bc-plane (cyan lines, Fig S3a-c). That is two oxygen atoms ( $\mathrm{O} 1$ and $\mathrm{O3}$ ) of a triflate anion are anchored to the neighboring methine and 5-pyrazolyl hydrogens ( $\mathrm{H} 7$ and $\mathrm{H} 13$, respectively) of a dication. The oxygen atom $\mathrm{O} 3$ participating in the interaction with the 5-pyrazolyl ( $\mathrm{H} 13)$ also interacts with a phenylene hydrogen, $\mathrm{H} 6$, situated ortho- to the phosphorus (and para- to the dipyrazolylmethyl) of a neighboring dication. A sheet parallel with the $b c$ - plane is formed (Fig S3b-c) as a result of the inversion symmetry of the dication that gives two $\mathrm{H} 6$ donors and two $\mathrm{O} 3$ acceptors per unit of $\mathbf{2}$. Acetonitrile is anchored to the sheet by a $\mathrm{CH} \cdots \mathrm{O}$ interaction ${ }^{(\mathrm{S2})}$ between the third oxygen, $\mathrm{O} 2$, of the triflate and a methyl hydrogen, $\mathrm{H} 2 \mathrm{Sb}$, of the solvate molecule as well as a $\mathrm{CH}$...Ninteraction ${ }^{(\mathrm{S2f}, \mathrm{S} 3)}$ between the nitrogen, $\mathrm{N} 1 \mathrm{~s}$, of $\mathrm{CH}_{3} \mathrm{CN}$ and the phenylene hydrogen, $\mathrm{H} 5$, that is positioned meta- to both the phosphorus and the $\mathrm{CHpz}_{2}$ moiety. Finally the $b c$ - sheets are stacked along the $a$ - direction by $\mathrm{C}$ $\mathrm{H} \cdots \pi$ interaction $^{(\mathrm{S4})}$ (red lines, Figs S3c and d) between a tolyl hydrogen, H33, ortho to the tolyl methyl) of one sheet and a pyrazolyl ring containing N41 of a neighboring sheet (the shortest contact is actually with C42 of the pz ring). 
$[\mathrm{Ag}(\mathrm{oL})](\mathrm{OTf}), 3$. Figure S4 illustrates the noncovalent interactions that govern the assembly of the polymer chains in $\mathbf{3}$ in three dimensions while Table S2 details the geometry of the (sub) van der Waals contacts. As described in the main text (see Fig. 4), $\mathbf{3}$ forms a coordination polymer that propagates

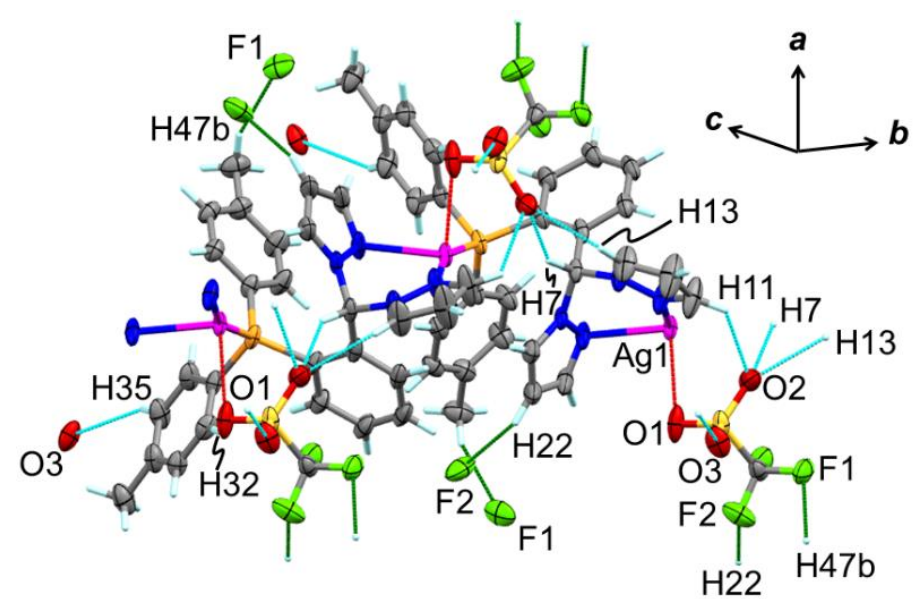

(a)

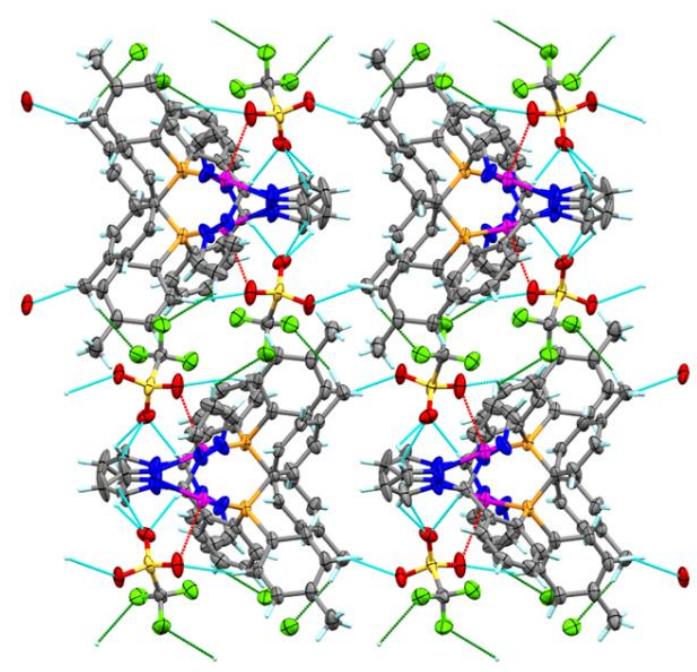

(c)

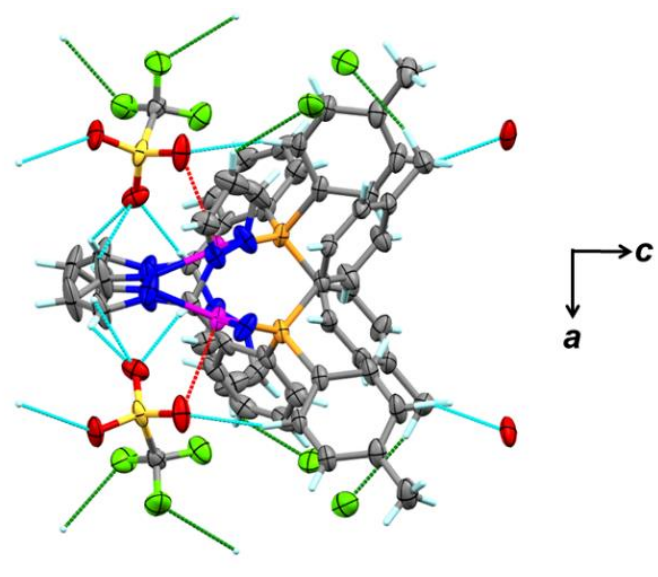

(b)

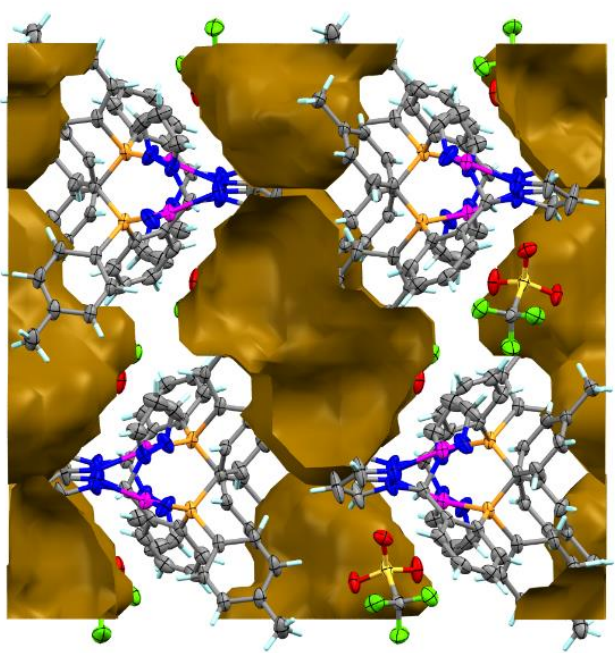

(d)

Figure S4. Supramolecular structure of $[\mathrm{Ag}(\mathrm{oL})](\mathrm{OTf})$, 3. (a) View of coordination polymer with short contacts ( $\mathrm{CH} \cdots \mathrm{O}$ cyan lines, $\mathrm{CH} \cdots \mathrm{F}$ green lines) and atom labeling. (b) View of coordination polymer down $b$-axis. (c) Representation of crystal packing diagram viewed down the $b$-axis with short noncovalent contacts highlighted as in (a). (d) View of one unit cell showing voids where disordered $\mathrm{Et}_{2} \mathrm{O}: \mathrm{CH}_{3} \mathrm{CN}$ reside. 
Table S2. Geometries of short $\mathrm{C}-\mathrm{H} \cdots\left(\mathrm{X}=\mathrm{O}, \mathrm{F}^{(\mathrm{SS})}\right)$ contacts in $[\mathrm{Ag}(\mathrm{oL})](\mathrm{OTf}), \mathbf{3}$.

\begin{tabular}{|c|c|c|c|c|}
\hline Donor(D)(-H) $\cdots$ Acceptor(A) & $\mathrm{D}-\mathrm{H}(\AA)$ & $H \cdots A(\AA)$ & $\mathrm{D} \cdots A(\AA)$ & $D-H \cdots A\left({ }^{\circ}\right)$ \\
\hline \multicolumn{5}{|c|}{$C-H \cdots O$ interactions $(\Sigma v d W-0.2 \AA)$} \\
\hline $\mathrm{C} 7-\mathrm{H} 7 \cdots \mathrm{O} 2$ & 1.00 & 2.25 & $3.117(3)$ & 144 \\
\hline $\mathrm{C} 11-\mathrm{H} 11 \cdots \mathrm{O} 2$ & 0.95 & 2.51 & $3.134(5)$ & 123 \\
\hline $\mathrm{C} 13-\mathrm{H} 13 \cdots \mathrm{O} 2$ & 0.95 & 2.43 & $3.146(4)$ & 132 \\
\hline $\mathrm{C} 32-\mathrm{H} 32 \cdots \mathrm{O} 1$ & 0.95 & 2.43 & $3.299(4)$ & 152 \\
\hline \multicolumn{5}{|c|}{$C-H \cdots O$ interactions $(\Sigma v d W-0.1 \AA$} \\
\hline $\mathrm{C} 35-\mathrm{H} 35 \cdots \mathrm{O}$ & 0.95 & 2.61 & $3.434(4)$ & 145 \\
\hline \multicolumn{5}{|c|}{$C-H \cdots F v d W$ limit contacts } \\
\hline $\mathrm{C} 47-\mathrm{H} 47 \mathrm{~b} \cdots \mathrm{F} 1$ & 0.98 & 2.58 & $3.509(4)$ & 159 \\
\hline $\mathrm{C} 22-\mathrm{H} 22 \cdots \mathrm{F} 2$ & 0.95 & 2.62 & $3.293(4)$ & 128 \\
\hline
\end{tabular}

with the $b$-axis. The triflate anion is bound to the cationic polymer backbone by a relatively long Ag $\cdots 0$ contact (Ag1-01 2.809(3) $\AA$, red lines, Figure S4a and Fig. 4, main text) and is held further by four short "intrachain" $\mathrm{CH} \cdots \mathrm{O}$ interactions that are $0.2 \mathrm{~A}$ less than the sum of the van der Waals radii of $\mathrm{H}$ and $\mathrm{O}$ (Table S2, cyan lines Fig. S4). That is $\mathrm{O} 2$ of the anion is involved in a trifurcated $\mathrm{CH} \cdots \mathrm{O}$ interaction with the methine and 5-pyrazolyl hydrogen atoms $(\mathrm{H7}, \mathrm{H} 13)$ of one monomer unit and with the 3-pyrazolyl hydrogen, $\mathrm{H} 11$, of the neighboring monomer unit in the polymer chain. The fourth intrachain $\mathrm{CH} \cdots \mathrm{O}$ interaction occurs with $\mathrm{O} 1$ (that is also in contact with $\mathrm{Ag}$ ) and a tolyl ring hydrogen that is ortho to phosphorus. The chains are connected along the c- direction to form sheets parallel with the bc-plane by a longer $(\Sigma \mathrm{vdW}-0.1 \AA$ ) C-H...O interaction between the third oxygen of the triflate, O3, of one chain and a tolyl ring hydrogen, $\mathrm{H} 35$, meta- to phosphorus of a neighboring chain. The $b c$ - sheets are stacked along the c- direction such that there are two short $\mathrm{CH} \cdots \mathrm{F}$ contacts (green lines, Fig. S4) that are just shorter than $2.67 \AA$, the sum of the van der Waals radii of $\mathrm{H}$ and $\mathrm{F}$. The first contact occurs between the triflate fluorine $\mathrm{F} 1$ and a tolyl methyl hydrogen, $\mathrm{H} 47 \mathrm{~b}$ while the second occurs between $\mathrm{F} 2$ and a 4pyrazolyl hydrogen, H22. This assembly leaves large voids (Fig. S4d) in the unit cell accounting for 1064 out of $8487 \AA^{3}$ or $16.4 \%$ of the total cell volume. Heavily disordered solvent mixture (approximately 1:1 $\mathrm{CH} 3 \mathrm{CN}$ Et2O) reside in the voids. These are easily removed on drying in air or under vacuum. 
Figure S5 labels the atoms and centroids of various short non-covalent interactions that organize the supramolecular structure of $\left[\left(m-\mathrm{IC}_{6} \mathrm{H}_{4} \mathrm{CHpz}_{2}\right)_{2} \mathrm{Ag}\right](\mathrm{OTf}), \mathbf{5}$; the metrics are listed in Table $\mathrm{S} 3$.
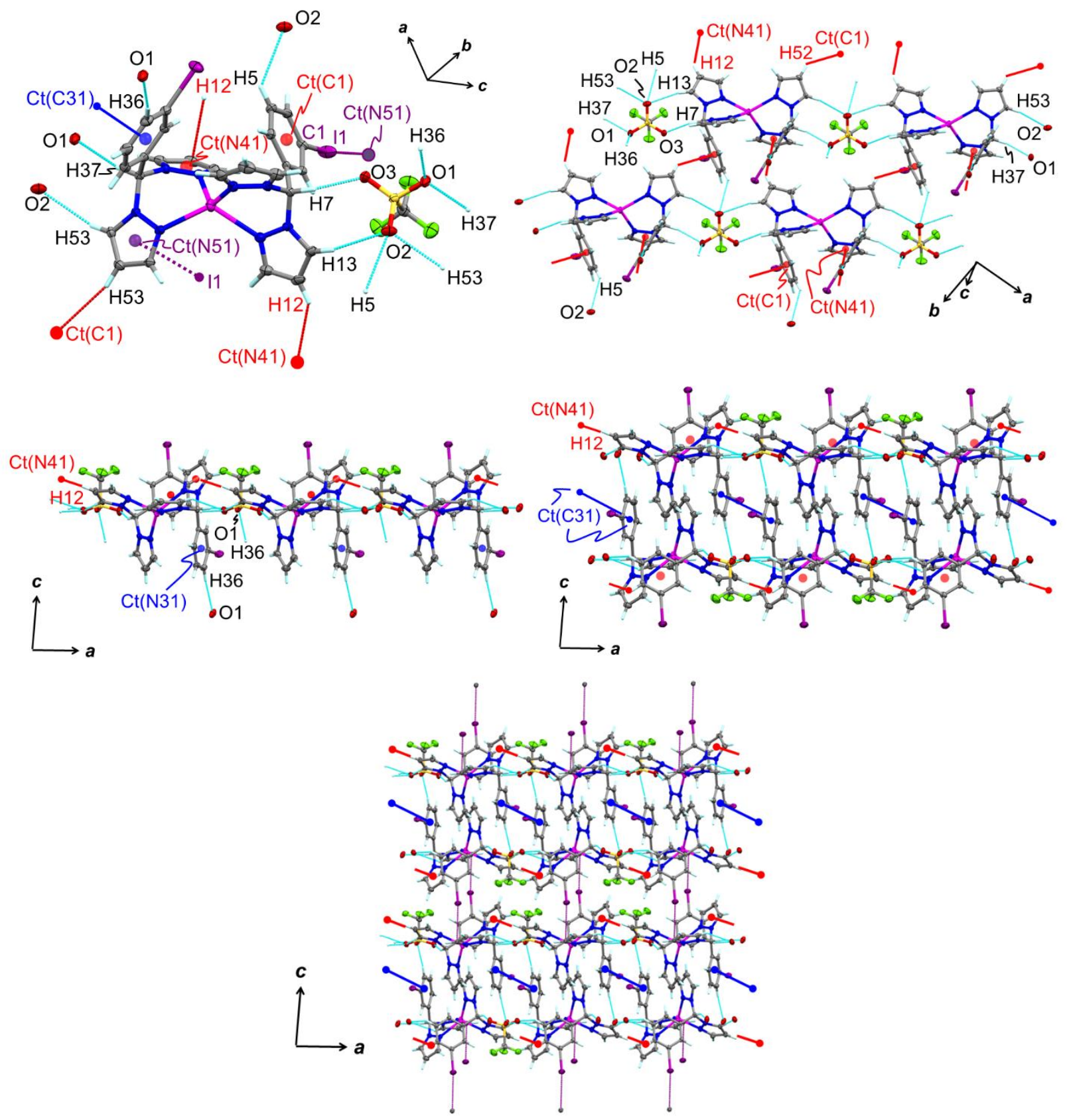

Figure S5. Supramolecular structure of $\left[\left(m-\mathrm{IC}_{6} \mathrm{H}_{4} \mathrm{CHpz}\right)_{2} \mathrm{Ag}\right](\mathrm{OTf}), 5$. Top left: View of asymmetric unit with labeling of important contacts ( $\mathrm{C}-\mathrm{H} \cdots \mathrm{O}$, cyan; $\mathrm{C}-\mathrm{H} \cdots \pi$, red; $\pi \cdots \pi$, blue; $\mathrm{C}-\mathrm{I} \cdots \pi$, purple). Top right: View of one layer perpendicular to $a b$ - plane. Middle left: View of one layer down $b$-axis. Middle right: View down $b$ - axis of bilayer sheet. Bottom: Stacking of two bilayer sheets giving short C-I $\cdots \pi$ contacts (purple vertical lines). 
Table S3. Summary of short contacts and non-covalent interactions organizing the supramolecular structure of $\left[\left(m-\mathrm{IC}_{6} \mathrm{H}_{4} \mathrm{CHpz}_{2}\right)_{2} \mathrm{Ag}\right](\mathrm{OTf}), \mathbf{5}$.

\begin{tabular}{|c|c|c|c|c|c|}
\hline$\pi \cdots \pi^{(\mathrm{S6})}$ & $\mathrm{Ct}-\mathrm{Ct}$ & $\perp_{\text {Avg }} \operatorname{Dist}(\AA)$ & $\alpha\left(^{\circ}\right)$ & $\beta\left(^{\circ}\right)$ & $\gamma\left(\left(^{\circ}\right)\right.$ \\
\hline $\mathrm{Ct}(\mathrm{C} 31)-\mathrm{Ct}(\mathrm{C} 31)$ & $3.816(2)$ & $3.472(1)$ & 0 & 24.5 & 24.5 \\
\hline Donor(D)-X ‥Acceptor(A) & $D-X(\AA)$ & $X \cdots A(\AA)$ & $D \cdots A(\AA)$ & $D-X \cdots A\left({ }^{\circ}\right)$ & $\gamma\left({ }^{\circ}\right)$ \\
\hline $\mathrm{C} 12-\mathrm{H} 12 \cdots \mathrm{Ct}(\mathrm{N} 41)$ & 0.95 & 2.80 & $3.585(3)$ & 140 & 13.0 \\
\hline $\mathrm{C} 52-\mathrm{H} 52 \cdots \mathrm{Ct}(\mathrm{C} 1)$ & 0.95 & 2.79 & $3.540(4)$ & 136 & 9.6 \\
\hline $\mathrm{C} 36-\mathrm{H} 36 \cdots \mathrm{O} 1$ & 0.95 & 2.39 & $3.332(4)$ & 173 & \\
\hline 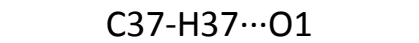 & 1.00 & 2.13 & $3.116(4)$ & 167 & \\
\hline $\mathrm{C} 5-\mathrm{H} 5 \cdots \mathrm{O} 2$ & 0.95 & 2.56 & $3.246(4)$ & 129 & \\
\hline $\mathrm{C} 13-\mathrm{H} 13 \cdots \mathrm{O} 2$ & 0.95 & 2.48 & $3.411(4)$ & 165 & \\
\hline $\mathrm{C} 53-\mathrm{H} 53 \cdots \mathrm{O} 2$ & 0.95 & 2.55 & $3.376(4)$ & 146 & \\
\hline $\mathrm{C} 7-\mathrm{H} 7 \cdots \mathrm{O} 3$ & 1.00 & 2.08 & $3.015(4)$ & 155 & \\
\hline 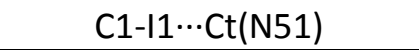 & $2.094(3)$ & $3.5580(13)$ & $5.616(3)$ & $166.61(8)$ & 12.3 \\
\hline \multicolumn{6}{|c|}{$\begin{array}{c}\mathrm{Ct}(i)=\text { centroid of ring containing atom } \mathrm{i} ; \alpha=\text { dihedral angle between mean planes } \mathrm{x} \text { and } \mathrm{y} . \beta= \\
\text { Angle } \mathrm{Ct}(i)-\mathrm{Ct}(j) ; \gamma=\text { Angle } \mathrm{Ct}(i)-\mathrm{Ct}(j) \text { and normal to plane containing } \mathrm{Ct}(j) ; \perp_{\text {Avg }} \text { Dist }=\text { average of } \\
\text { perpendicular distance of centroid } i \text { to ring } j \text { and of centroid } j \text { to ring } i .\end{array}$} \\
\hline
\end{tabular}

There are six $\mathrm{C}-\mathrm{H} \cdots \mathrm{O}$ interactions (cyan lines Fig. S5) that hold triflate anions to cations. Five of the C$\mathrm{H} \cdots \mathrm{O}$ interactions assemble ions into a sheet structure that lies parallel with the $a b$ - plane. Thus, neighboring methine and 5-pyrazolyl hydrogens ( $\mathrm{H} 7$ and $\mathrm{H} 13$, respectively) serve as donors to $\mathrm{O} 3$ and $\mathrm{O} 2$ acceptors, respectively, of a triflate anion, attaching ions by two points. The ion pair is assembled into a polymer cahin along the a- direction by a similar set of $\mathrm{C}-\mathrm{H} \cdots \mathrm{O}$ interactions involving the methine (H37) and 5-pyrazolyl (H53) hydrogens on the other side of the cation which interact with $\mathrm{O} 1$ and $\mathrm{O} 2$ of a neighboring ion pair. The polymer chains are assembled along $b$-into a sheet structure by the interaction between $\mathrm{O} 2$ and the phenylene hydrogen ( $\mathrm{H} 5$ ) meta- to both the iodine atom, 11 , and the $\mathrm{CHpz}_{2}$ group (top right Fig. S5). Two $\mathrm{CH}-\pi$ interactions between two 4-pyrazolyl donors ( $\mathrm{H} 12$ and $\mathrm{H} 52$ ) and a pyrazolyl (containing N41) or an iodophenyl (containing C1) further support the sheet structure. Two sheets are held together into a bilayer by the sixth $\mathrm{C}-\mathrm{H} \cdots \mathrm{O}$ interaction between $\mathrm{O} 1$ of one sheet and a phenylene hydrogen, $\mathrm{H} 35$, that is ortho- to iodine (12) but para- to a $\mathrm{CHpz}_{2}$ moiety on an neighboring sheet. A $\pi-\pi$ interaction $^{(S 6)}$ (blue lines, Fig S5) between two iodophenyl groups that contain 12 further supports the bilayer. The bilayers are stacked along the $c$ - axis in manner such that there is a short contact (purple lines Fig S5) between I1 and a pyrazolyl carbon C51 of $3.50 \AA$ that is nearly $0.2 \AA$ shorter than $3.68 \AA$, the sum of the van der Waals radii of $C$ and I. 

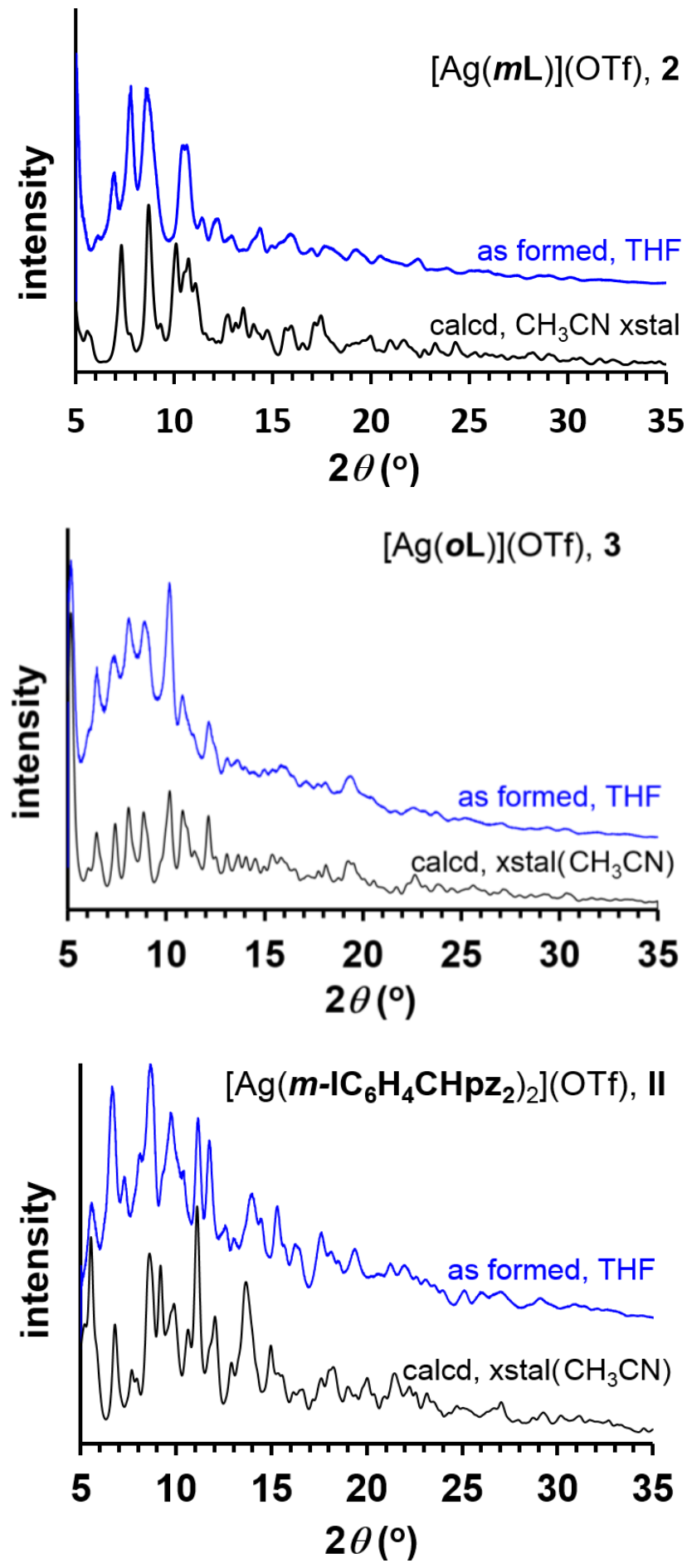

Figure S6. X-ray Powder Diffraction Patterns for samples of samples of $\mathbf{2}, \mathbf{3}$, and $\mathbf{5}$ obtained directly after work-up (prior to recrystallization) and comparison with patterns calculated from single crystal $\mathrm{X}$ ray diffraction studies. 


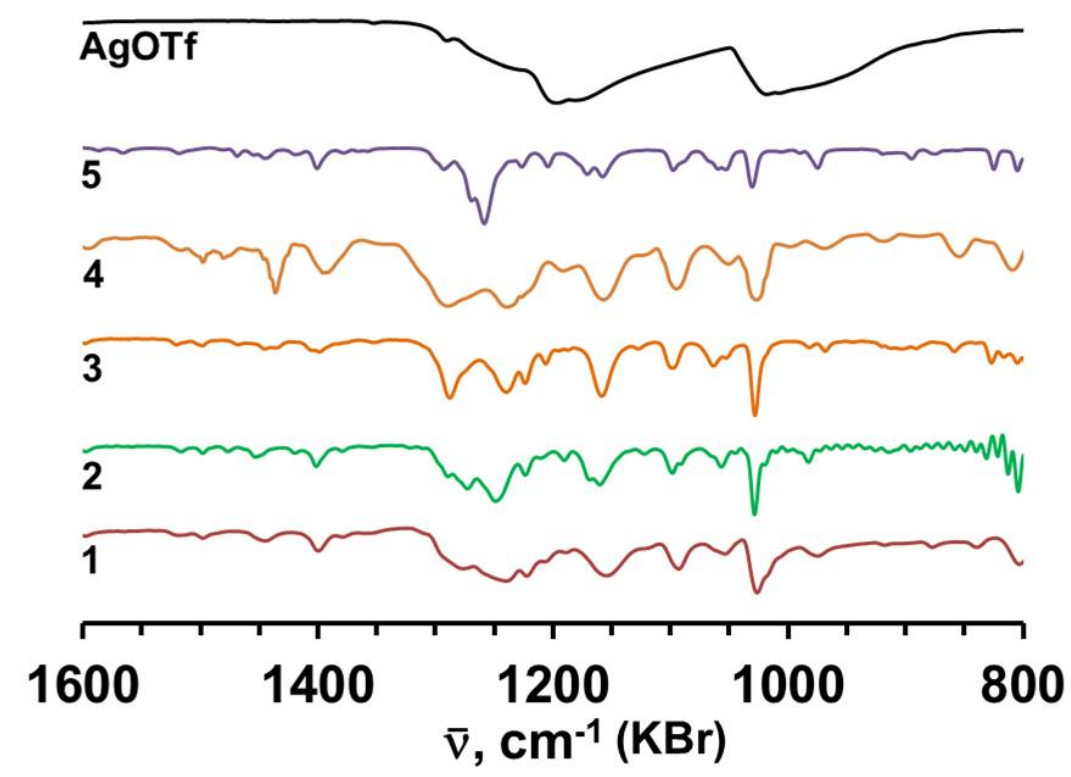

Figure S7. Portion of the IR spectrum obtained for solid samples of 1-4, and $\mathbf{5}$ as $\mathrm{KBr}$ pellets. 

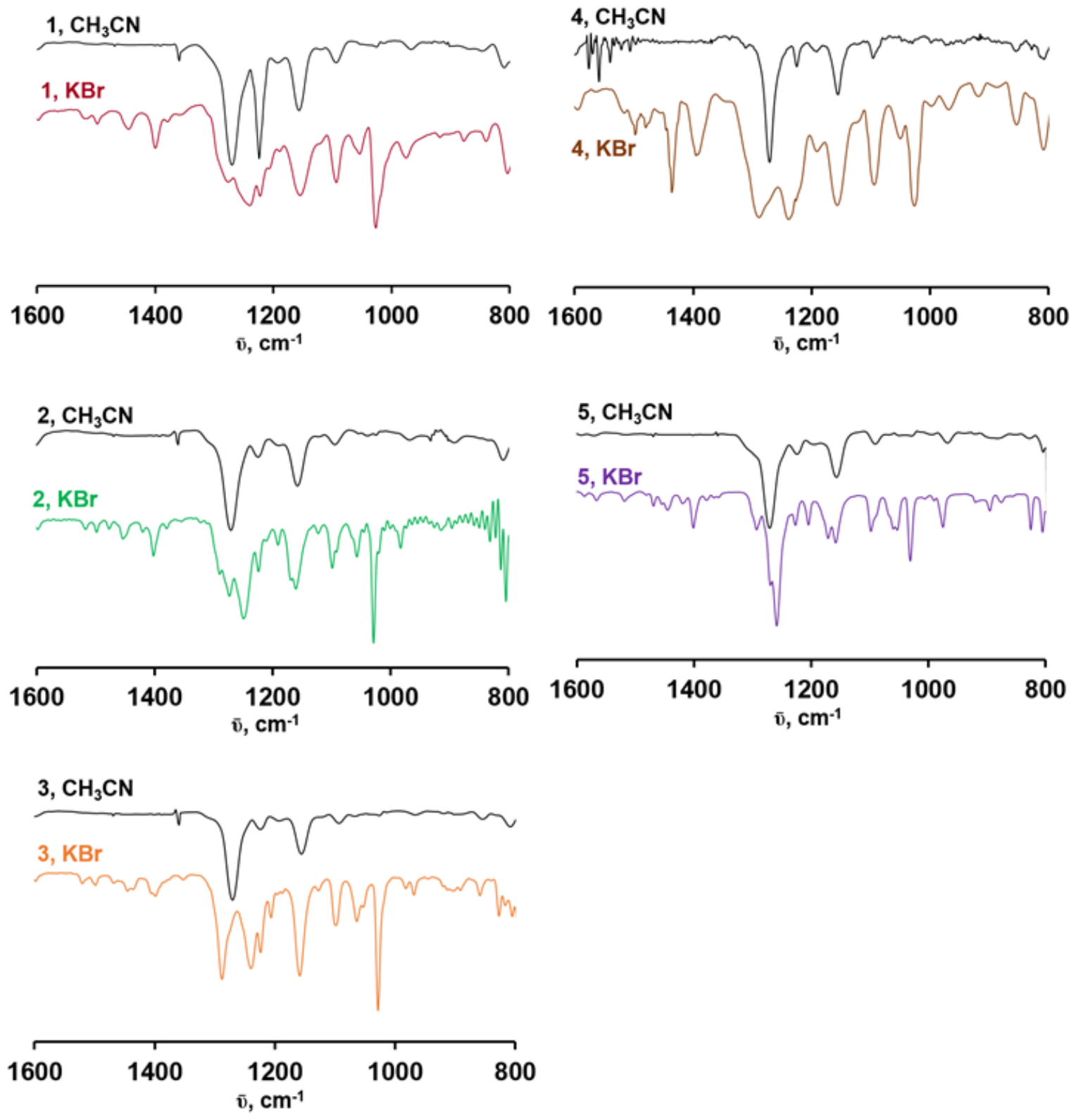

Figure S8. Comparison of portion (in the S-O and C-F stretching region) of IR spectra of each 1-5 in $\mathrm{CH}_{3} \mathrm{CN}$ versus solid samples. 


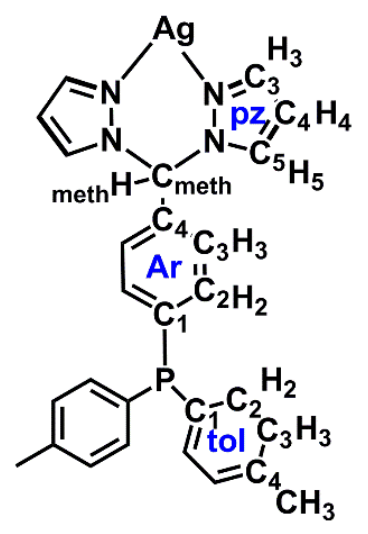

(1)

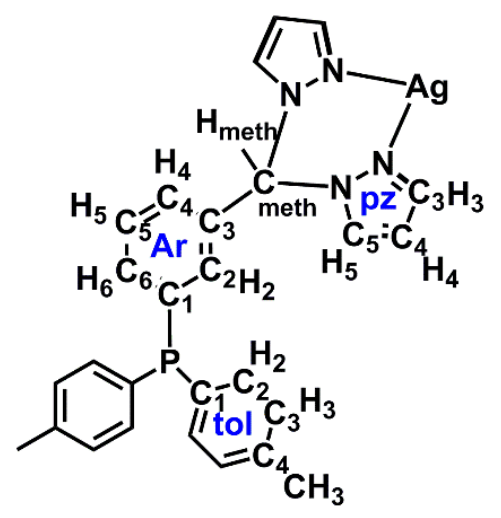

(2)

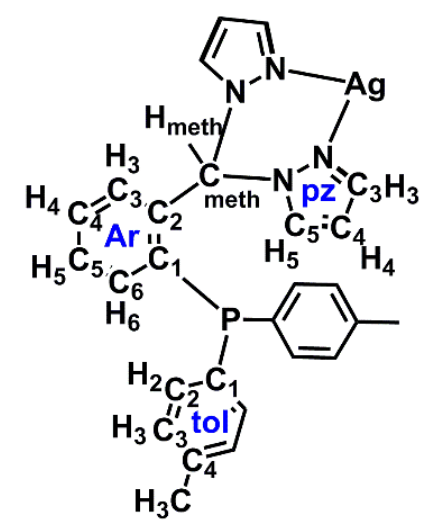

(3)

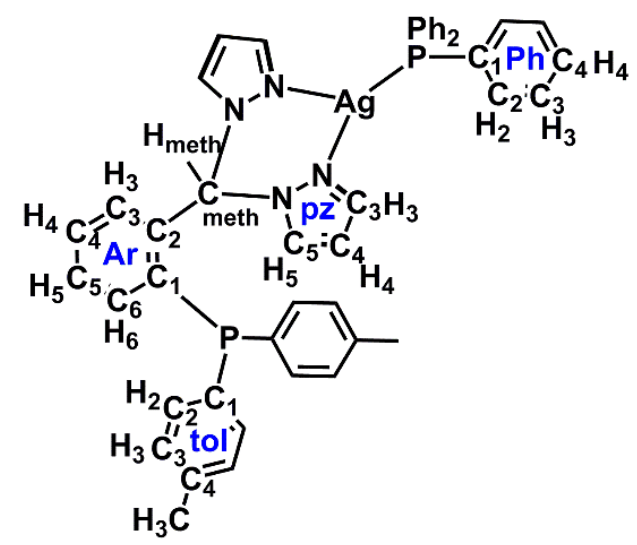

(4)

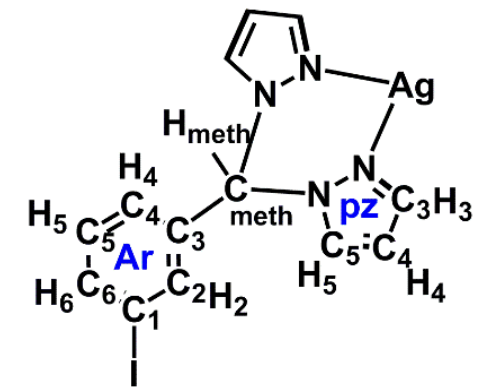

(5)

Figure S9. Atom labeling diagrams of 1-5 used in experimental section for NMR assignments. 

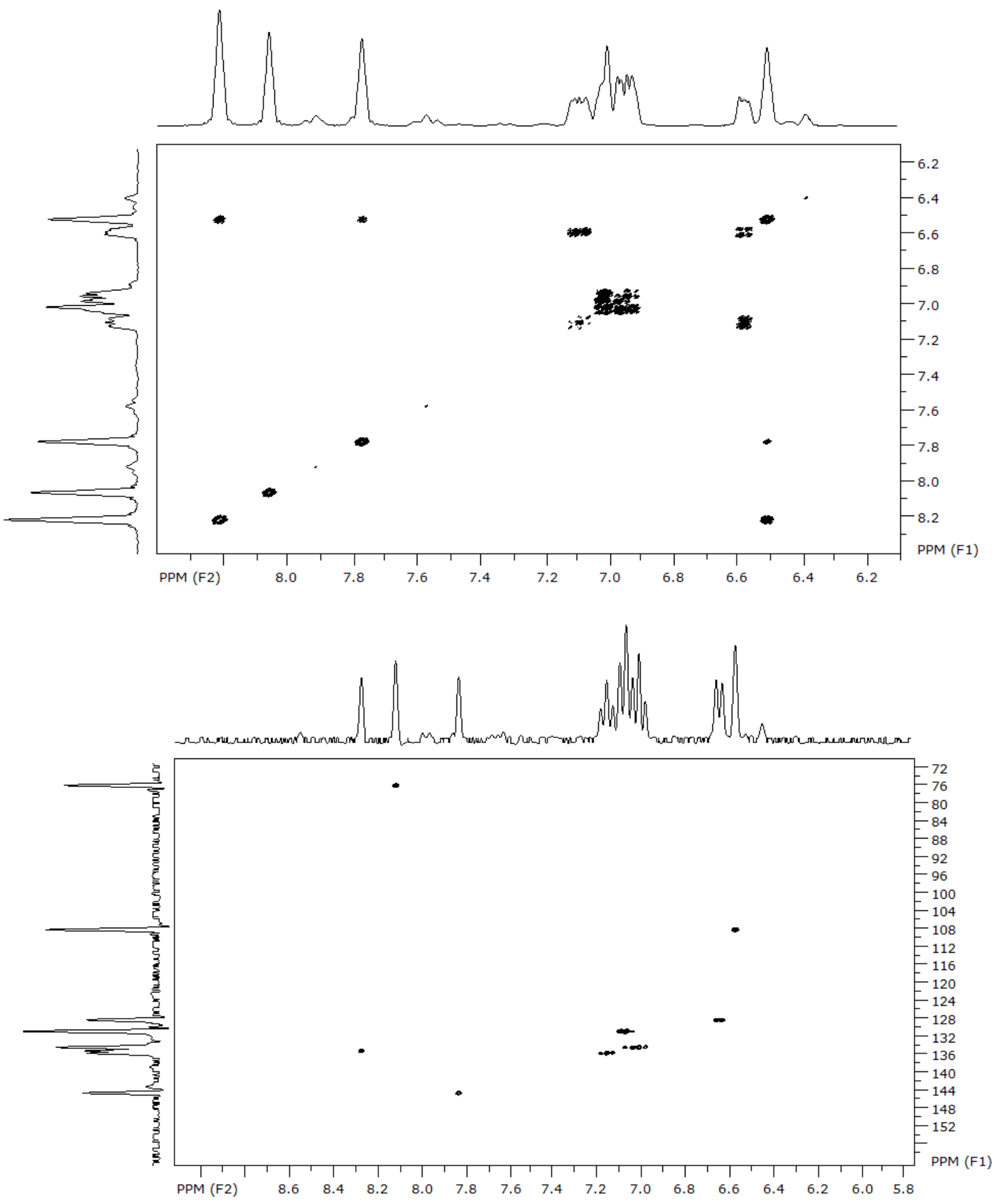

Figure S10. COSY (top) and HMQC (bottom) spectra of 1 in $\mathrm{CD}_{3} \mathrm{CN}$. 


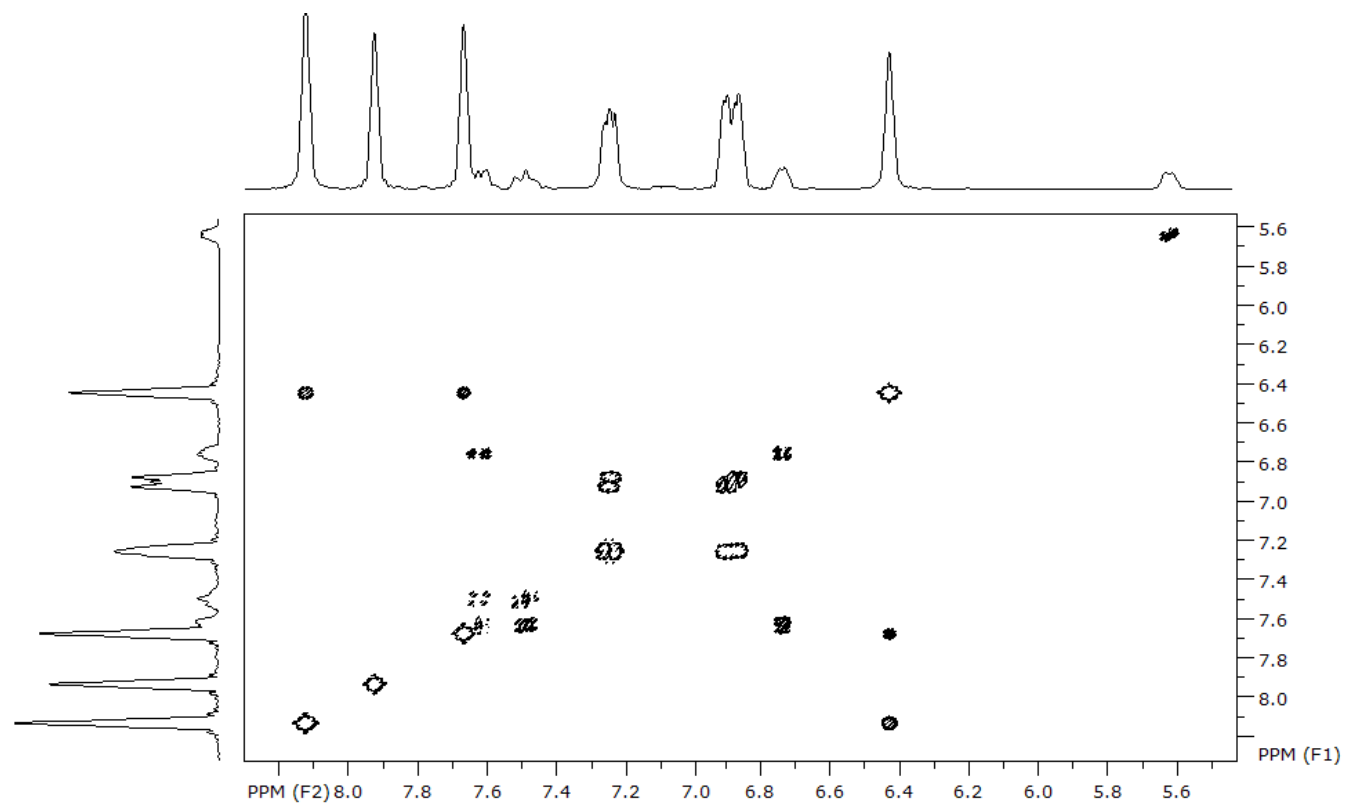

SpinWorks 3:

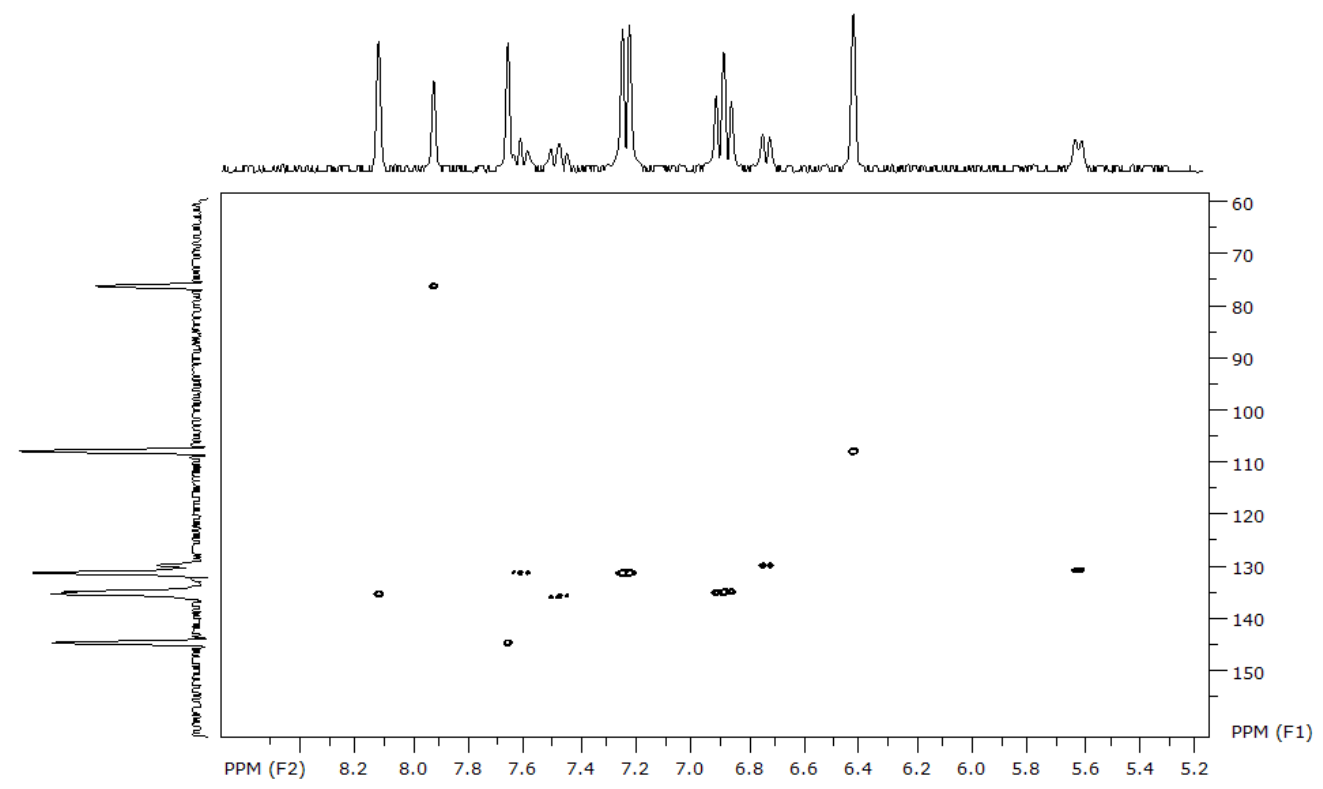

Figure S11. COSY (top) and HMQC (bottom) NMR spectrum of 2 in $\mathrm{CD}_{3} \mathrm{CN}$. 

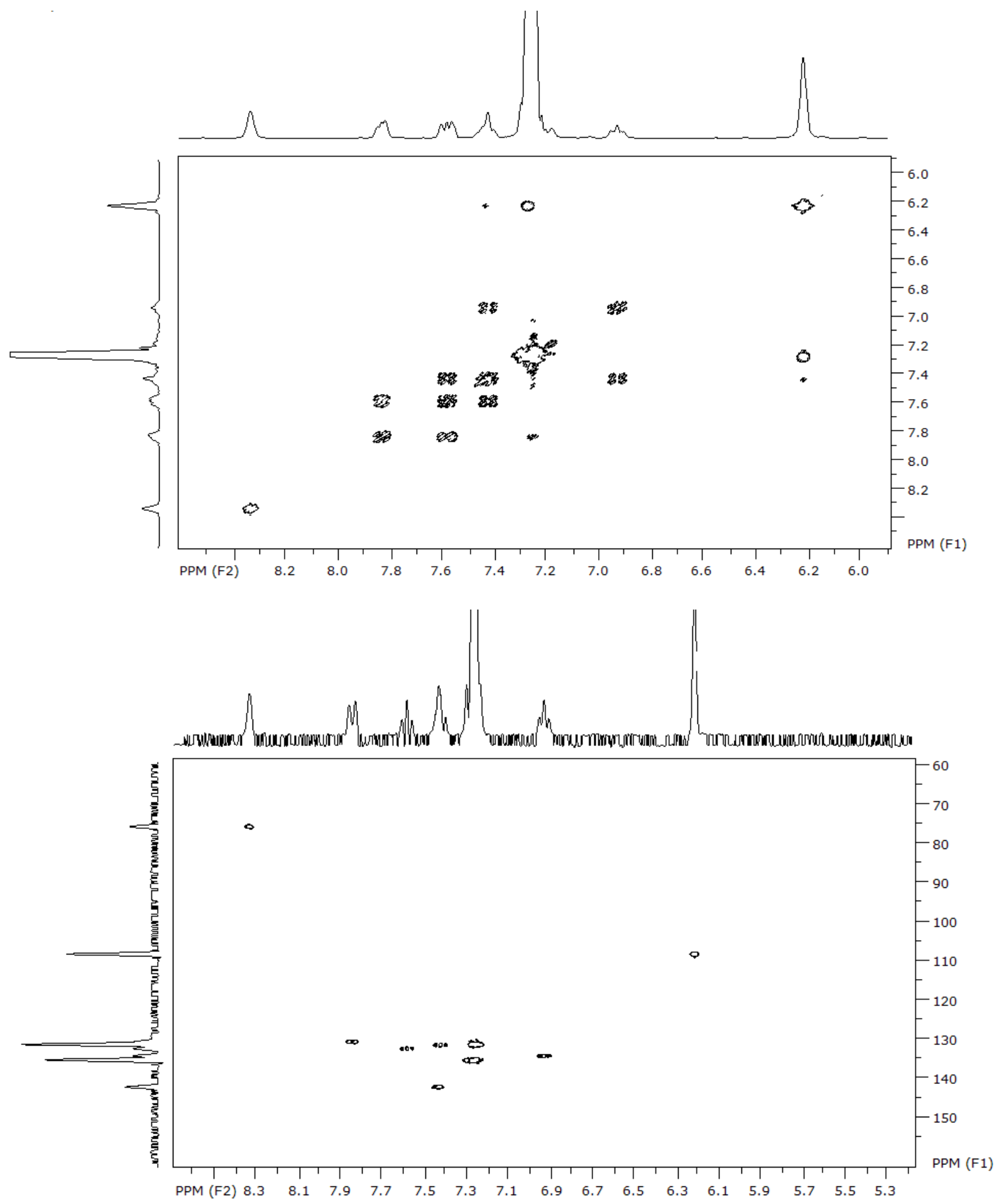

Figure S12. COSY (top) and HMQC (bottom) NMR spectrum of $\mathbf{3}$ in $\mathrm{CD}_{3} \mathrm{CN}$. 


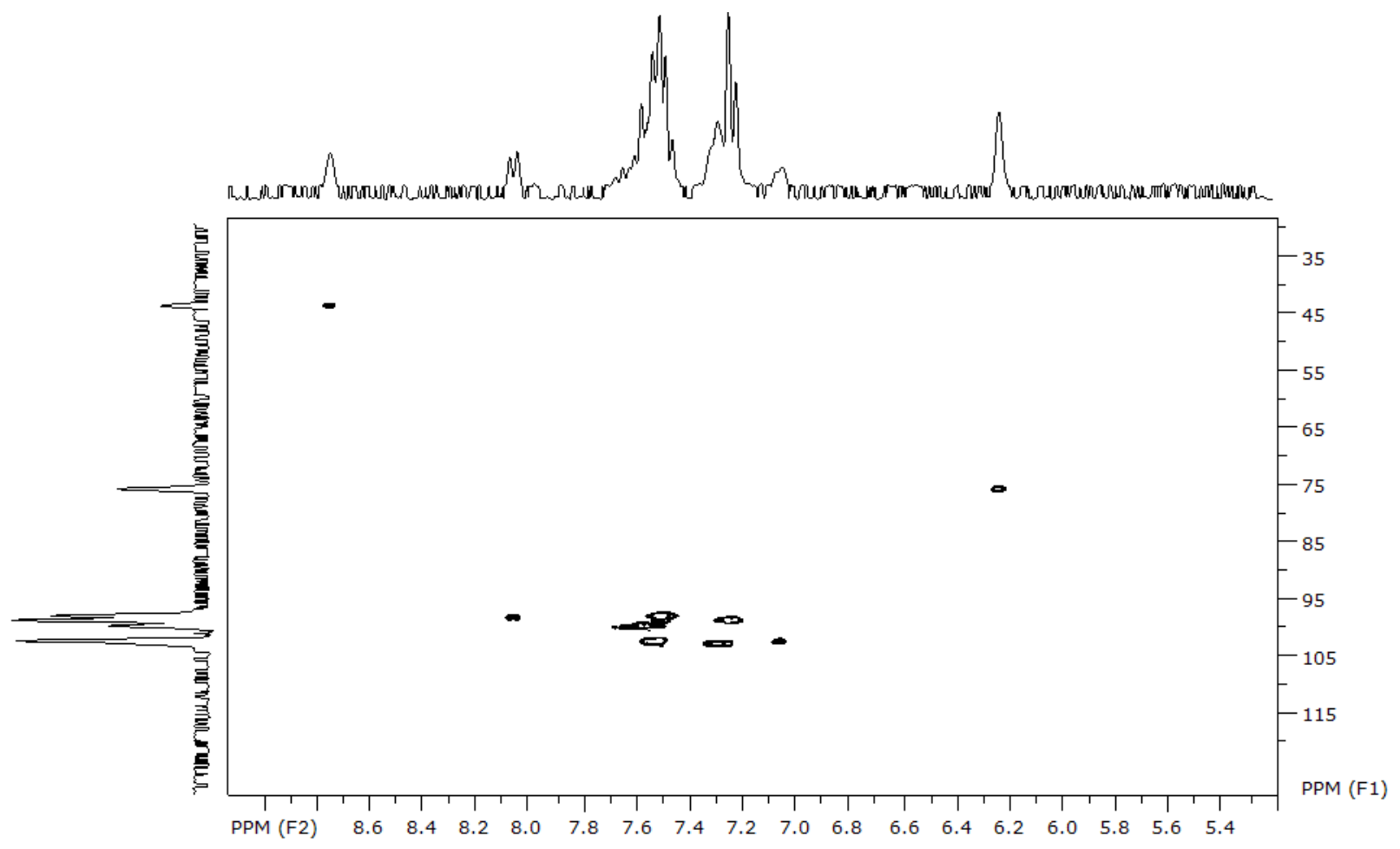

Figure S13. $\mathrm{HMQC} N M R$ spectrum of 4 in $\mathrm{CD}_{3} \mathrm{CN}$. 

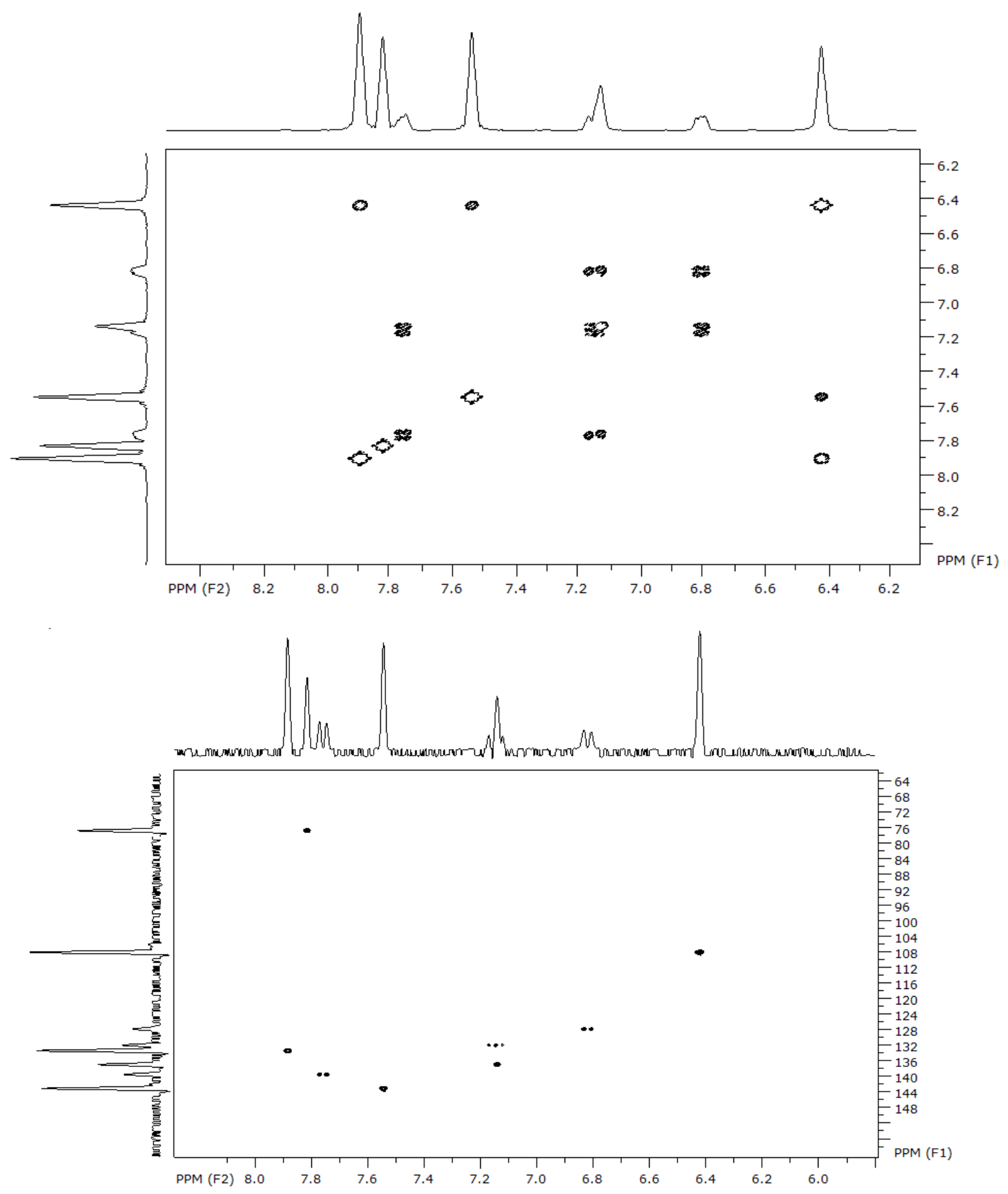

Figure S14. COSY (top) and HMQC (bottom) NMR spectrum of 5 in $\mathrm{CD}_{3} \mathrm{CN}$. 


\section{References.}

(S1) CSD User Manuals; Cambridge Crystallographic Data Centre, 12 Union Road, Cambridge CB2 1EZ, U.K.

(S2) (a) Wheeler, S. E.; Bloom, J. W. G. J. Phys. Chem. A 2014, Advance Article. (b) Bernstein, J. Cryst. Growth Des., 2013, 13, 961-964. (c) Gu, Y.; Kar, T.; Scheiner, S. J. Am. Chem. Soc., 1999, 121, 9411-9422. (d) Desiraju, G. J. Acc. Chem. Res., 1996, 29, 441-449. (e) Steiner, T.; Saenger, F. J. Am. Chem. Soc., 1992, 114, 10146-10154. (f) Desiraju, G. J. Acc. Chem. Res., 1991, 24, 290-296. (f) Taylor, R.; Kennard O. J. Am. Chem. Soc. 1982, 104, 5063-5070.

(S3) (a) Sammes, M. P.; Harlow, R. L.; Simonsen, S. H. J. Chem. Soc., Perkin Trans. 2, 1976, 10, 1126-30. (b) Wu, D. Y.; Ren, Y.; Wang, X.; Tian, A. M.; Wong, N. B.; Li, Wai-Kee J. Molec. Struct. THEOCHEM 1999, 459, 171-176. (c) Dhumal, N. R.; Gejji, S. P. Chem. Phys. Lett., 2004, 397, 185-193.

(S4) (a) Takahashi, H.; Tsuboyama, S.; Umezawa, Y.; Honda, K.; Nishio, M. Tetrahedron, 2000, 56, 6185-6191. (b) Tsuzuki, S.; Honda, K.; Uchimaru, T.; Mikami, M.; Tanabe, K. J. Am. Chem.Soc., 2000, 122, 11450-11458. (c) Nishio, M.; Hirota, M.; Umezawa, Y. The CH- $\pi$ interaction: evidence, nature, and consequences, Wiley-VCH, Inc: New York, 1998. (d) Umezawa, Y.; Tsuboyama, S.; Honda, K.; Uzawa, J.; Nishio, M. Bull. Chem. Soc. Jpn 1998, 71, 1207-1213. (e) Braga, D.; Grepioni, F.; Tedesco, E. Organometallics 1998, 17, 2669-2672.

(S5) (a) Choudhury, A. R.; Row, T. N. G. Cryst. Growth Des., 2004, 4, 47-52. (b) van den Berg, J. A.; Seddon, K. R., Cryst. Growth Des. 2003, 3, 643. (c) Brammer , L.; Bruton E. A.; Sherwood, P. New J. Chem. 1999, 23, 965-968. (d) Grepioni, F.; Cojazzi, G.; Draper, S. M.; Scully, N.; Braga, D.

Organometallics 1998, 17, 296-307. (e) Thalladi , V. R.; Weiss , H. -C.; Bläser , D.; Boese , R.; Nangia, A.; Desiraju, G. R.; J. Am. Chem. Soc., 1998, 120, 8702-8710. 
(S6) (a) Salonen, L. M.; Ellermann, M.; and Diederich, F. Angew. Chem. Int. Ed. 2011, 50, 4808 - 4842.

(b) Lee, E. C.; Kim, D.; Jurečka, P.; Tarakeshwar, P.; Hobza, P.; Kim, K. S.; J. Phys. Chem. A 2007, 111, 3446-3457. (c) Janiak, C. J. Chem. Soc., Dalton Trans. 2000, 3885-3896. (d) McGaughey, G. B.; Gagné, M.; Rappé, A. K. J. Biol. Chem. 1998, 273, 15458-15463. (e) Hunter, C. A.; Sanders, J. K. M. J. Am.

Chem. Soc. 1990, 112, 5525-5534 